\title{
CHARACTERIZATION OF PRESSURE DRIVEN AND ELECTRO- KINETICALLY DRIVEN FLOW IN A MICRO-FLUIDIC CHIP USING PARTICLE IMAGING VELOCIMETRY
}

\author{
A Thesis \\ Presented to \\ the Faculty of \\ California Polytechnic State University, \\ San Luis Obispo \\ In Partial Fulfillment \\ of the Requirements for the Degree \\ Master of Science in Engineering
}

\author{
By \\ Alexis Weckel \\ June, 2015
}


(C) 2015

Alexis Weckel

ALL RIGHTS RESERVED 


\section{COMMITTEE MEMBERSHIP}

TITLE:

AUTHOR:

DATE SUBMITTED:

June, 2015
Characterization of Pressure Driven and ElectroKinetically Driven Flow in a Micro-Fluidic Chip Using Particle Imaging Velocimetry

\author{
Alexis Weckel
}

COMMITTEE CHAIR: Dr. Richard Savage Department Chair, Biomedical Engineering

COMMITTEE MEMBER: Dr. David Clague

Professor, Biomedical Engineering

COMMITTEE MEMBER: Dr. Lilly Laiho

Professor, Biomedical Engineering 


\begin{abstract}
Characterization of Pressure Driven and Electro-Kinetically Driven Flow in a Micro-Fluidic Chip Using Particle Imaging Velocimetry
\end{abstract}

\title{
Alexis Weckel
}

The flow profiles of pressure-driven and electro-kinetic driven flows were compared for a microfluidic chip. It was found that the pressure-driven flow had a parabolic profile while the electro-kinetic flow had a plug shaped flow profile. The measured velocities were similar to those determined by the Poiseuille flow model and the Helmholtz-Smoltchowski equation. Flow uniformity is very important for control in microfluidic mixers. Parabolic flow profiles lead to inconsistent reactions while the more uniform plug shape flow allow for a more steady reaction across the channel. Previous work had been performed to measure the flow of a solution of fluorescent polystyrene beads in PDMS channels using a laser confocal microscope. This showed that particles easily stuck to the channel making it difficult to measure over time. In addition, bubble formation in the channel made measuring velocities difficult. Current work used a LabSmith Video Synchronized microscope with software to measure the flow rates at different areas of the channel. Solutions of fluorescent polystyrene beads were used to visually observe the flow within a channel under a microscope. Four different channels were used for the pressure-driven flows of varying dimensions and materials. The channel with the best measured profile was also measured under electro-kinetic flow. A LabSmith High Voltage Sequencer was used to apply a voltage across the channel for electro-kinetic measurements. This 
research confirmed the different flow profiles under pressure-driven and electrokinetic driven flow. Future work can be done to determine how this effects mixing in the channels.

KEYWORDS: micro-fluidics, electro-kinetics, particle imaging velocimetry, flow profile, pressure-driven, electro-osmosis, electrophoresis 


\section{ACKNOWLEDGMENTS}

I would like to thank the following people for supporting my research:

Dr. Savage

Dr. Laiho

Dr. Clague

Professor Mayer

Nash Anderson

Brooke Ziegler

Sam Jade

Harry Lafferty
Department Chair, Biomedical Engineering

Professor, Biomedical Engineering

Professor, Biomedical Engineering

Professor, Mechanical Engineering

Alumnus, Materials Engineering

Student, Biomedical Engineering

Alumnus, Materials Engineering

Alumnus, Materials Engineering 


\section{TABLE OF CONTENTS}

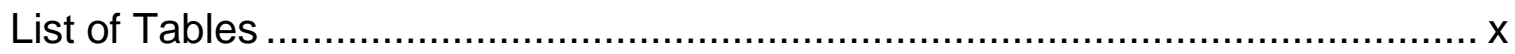

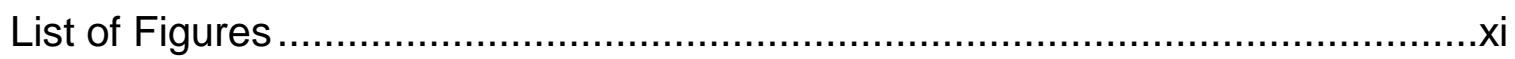

List of Equations ……..........................................................................

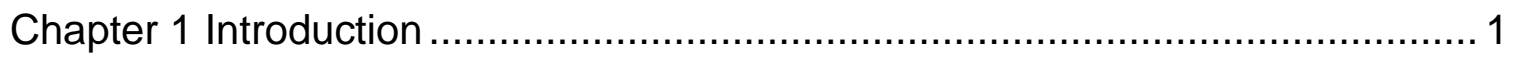

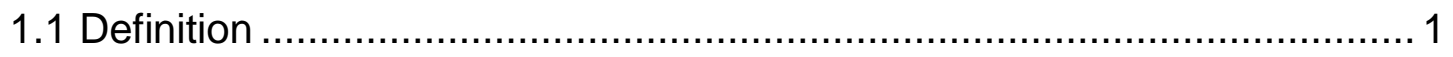

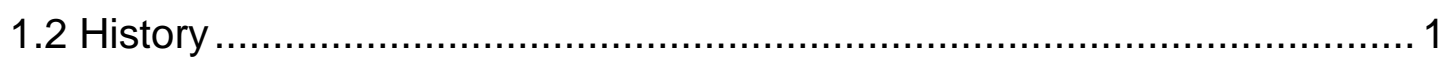

1.3 Project Definition/Research Question ............................................. 2

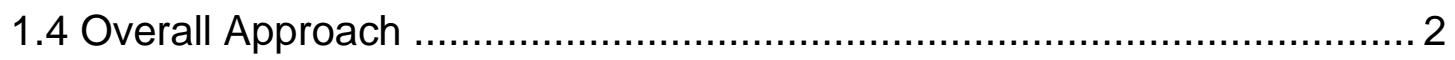

Chapter 2 Background \& Literature Review .................................................. 4

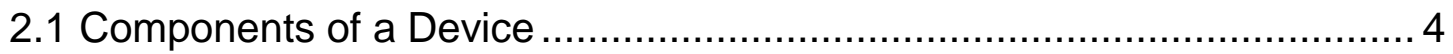

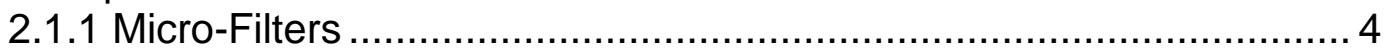

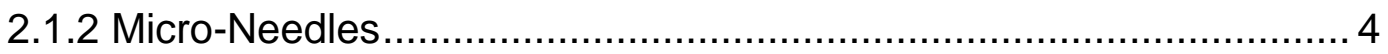

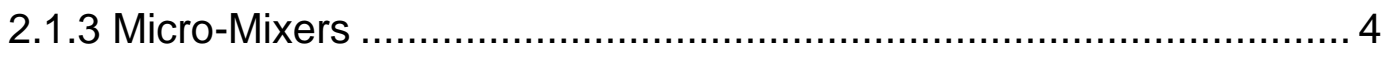

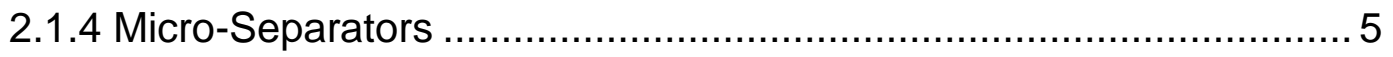

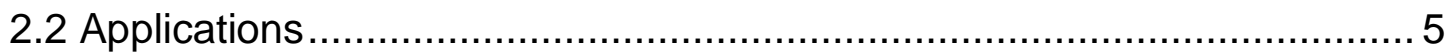

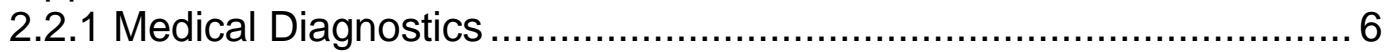

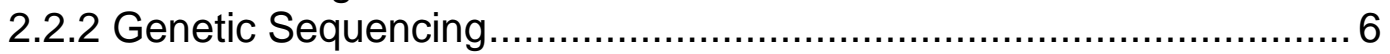

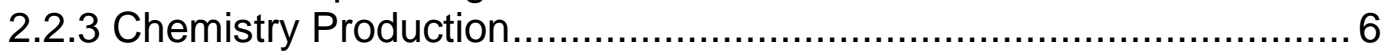

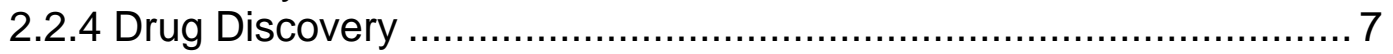

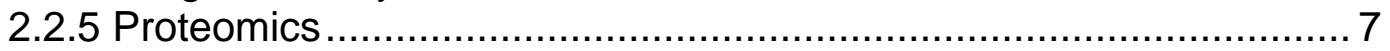

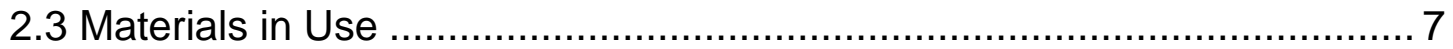

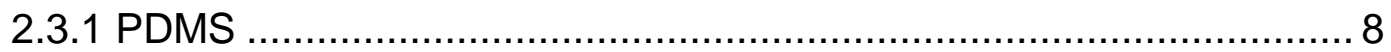

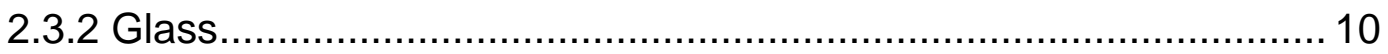

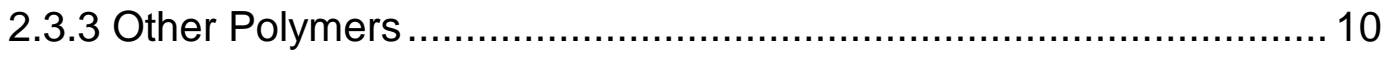

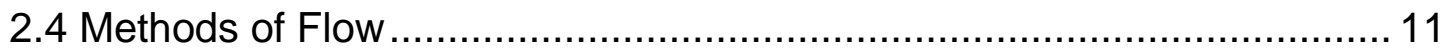

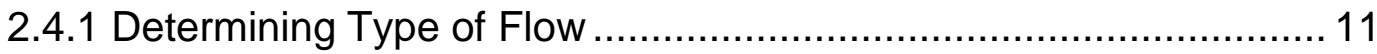

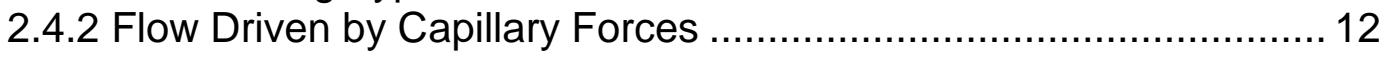

2.4.3 Pressure Driven Flow................................................................. 14

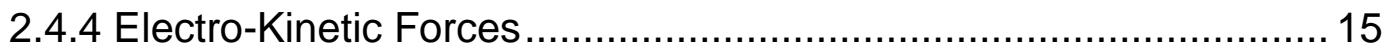

Streaming Potential ................................................................. 16

Sedimentary Potential ............................................................... 17

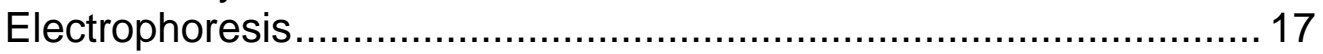

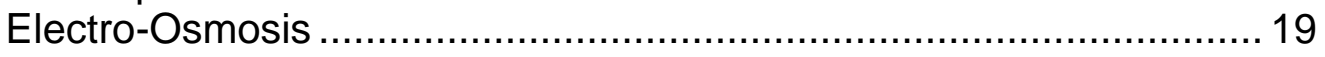




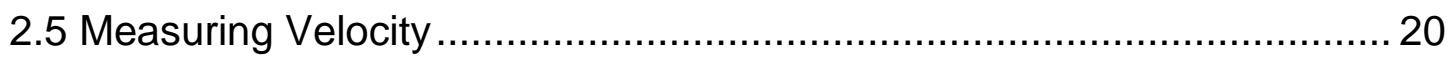

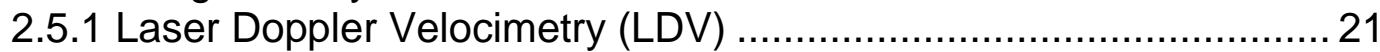

2.5.2 Molecular Tagging Velocimetry (MTV) .......................................... 22

2.5.3 Particle Image Velocimetry (PIV) .............................................. 22

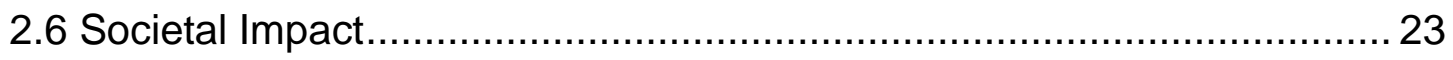

Chapter 3 Foundational Experiments …................................................... 24

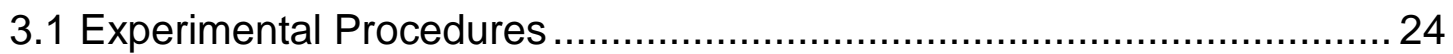

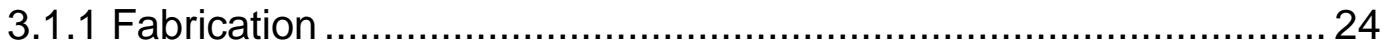

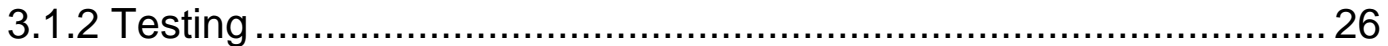

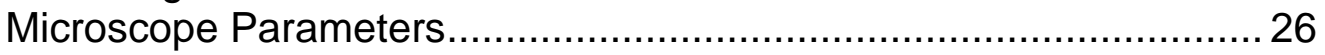

Pressure-Driven Flow Setup........................................................ 26

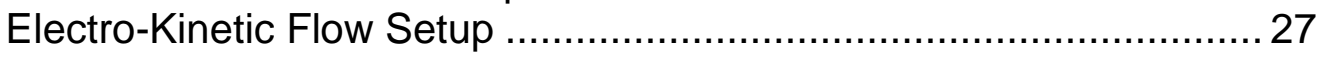

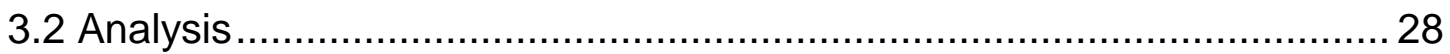

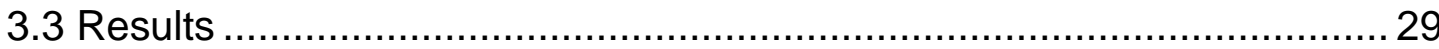

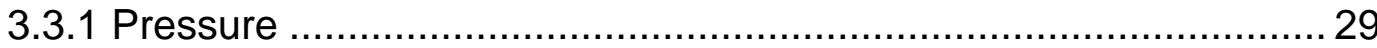

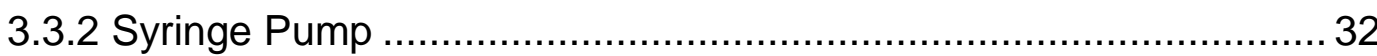

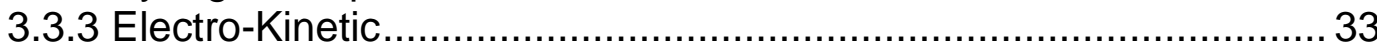

3.4 Lessons Learned from Foundational Experiment ................................... 34

Chapter 4 Materials, Methods, and Procedures .............................................. 36

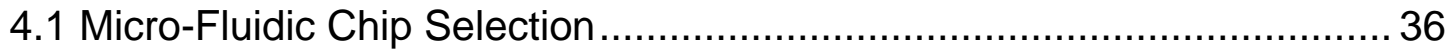

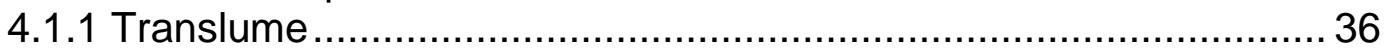

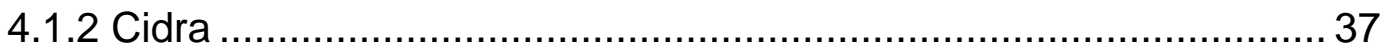

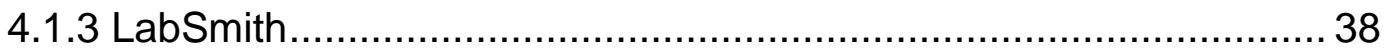

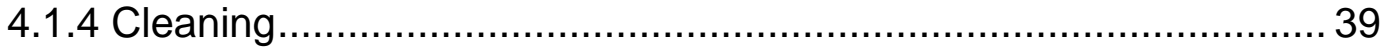

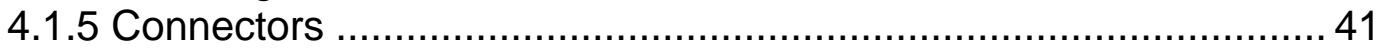

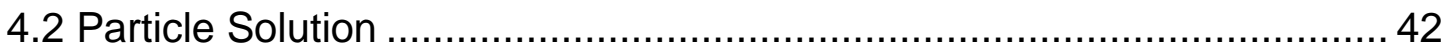

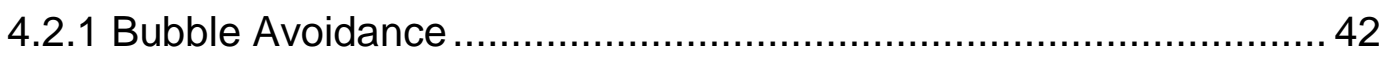

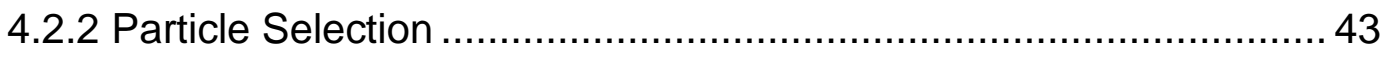

4.3 Pressure-Driven Flow Experimental Procedures.................................. 45

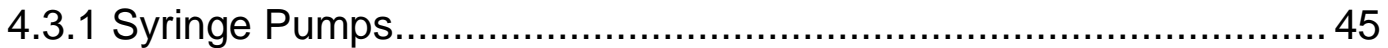

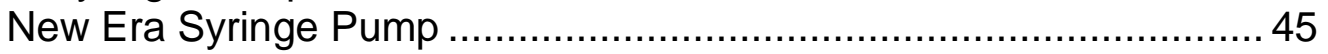

Harvard Apparatus Syringe Pump ................................................ 46

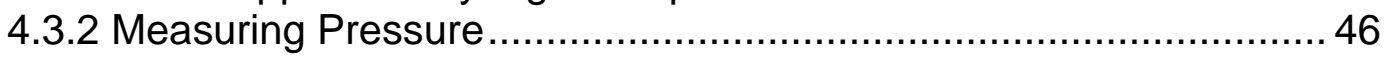

4.3.3 Measuring Velocity Profiles.................................................... 56

4.4 Electro-Kinetic Driven Flow Experimental Procedures .......................... 58

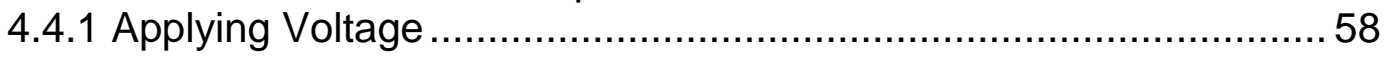

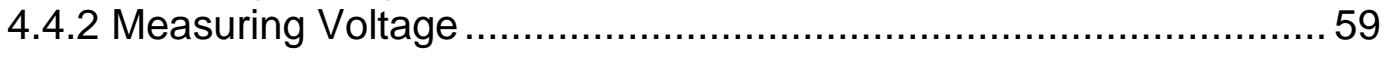




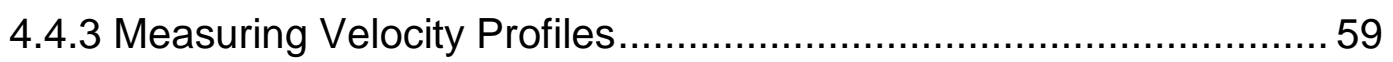

Chapter 5 Results, Analysis, and Discussion ............................................. 61

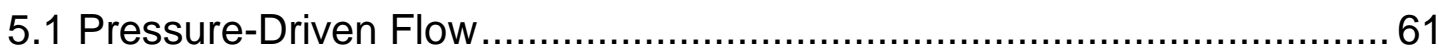

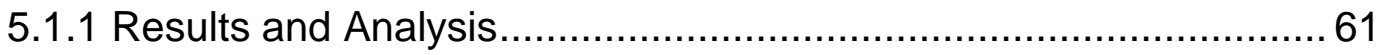

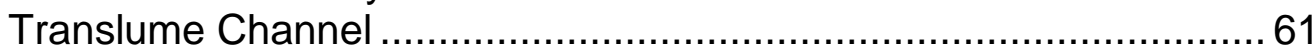

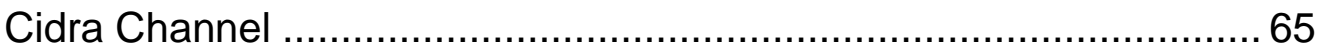

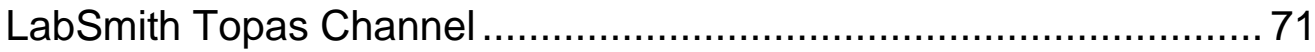

LabSmith PMMA Channel ............................................................. 77

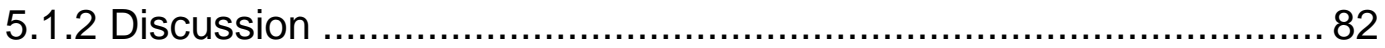

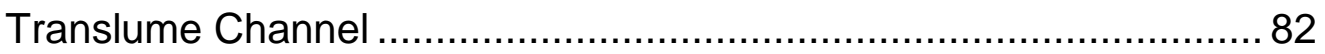

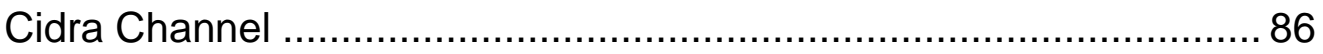

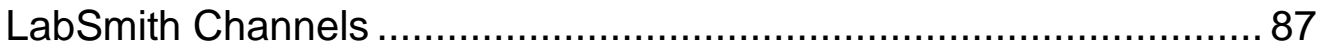

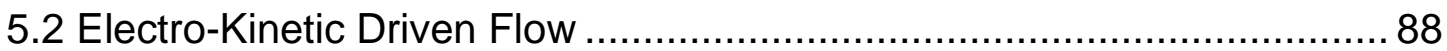

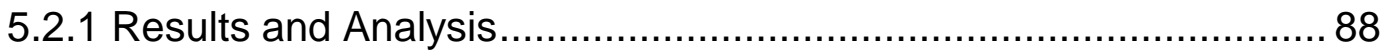

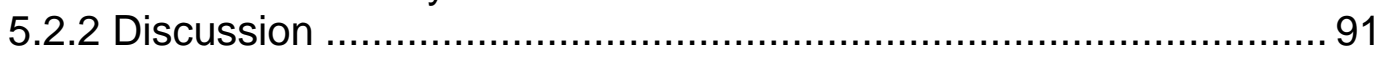

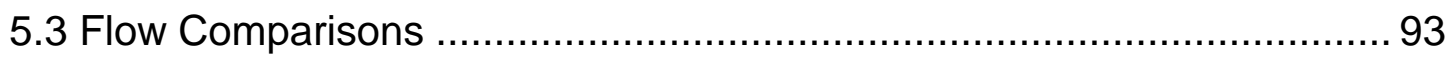

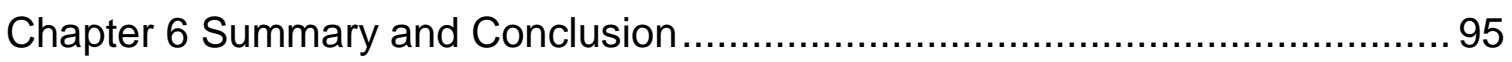

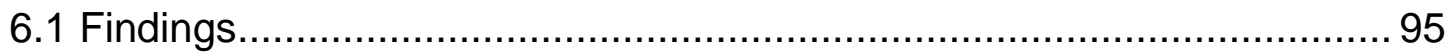

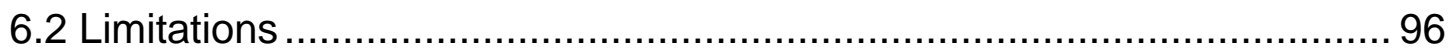

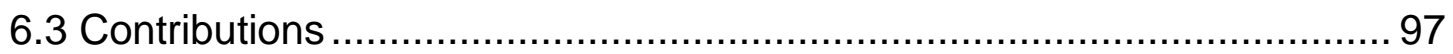

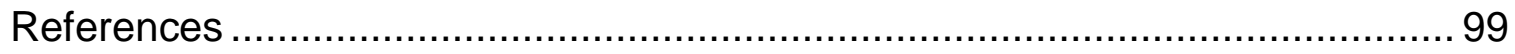

Appendices

A. PDMS Wetting Agent 564 by Cidra Precision Services .......................... 104

B. LabSmith Synchronized Video Microscope.......................................... 107

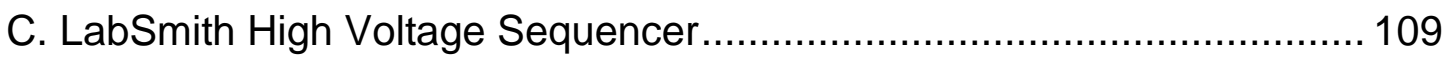

D. Modeling Pressure-Driven Flow at Varying Flow Rates......................... 110

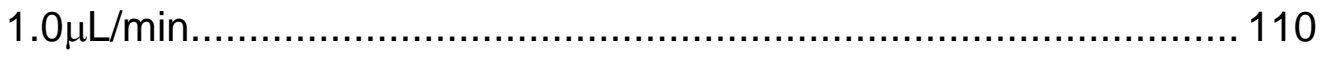

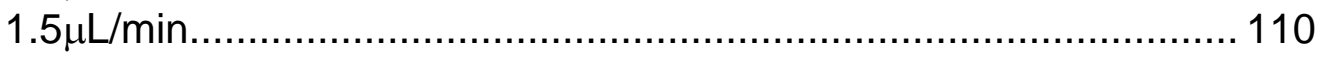

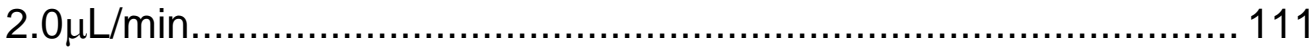

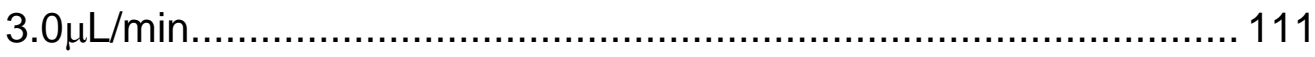

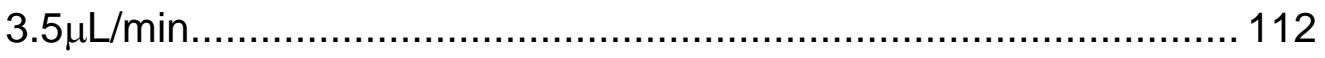

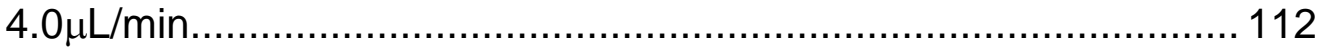




\section{LIST OF TABLES}

Table I. Electro-kinetic force comparison chart. The four main types of electrokinetic forces can be categorized by the movement of solids and liquids in the system and what is applied to the system.

Table II. Pressure-driven results summary by laser confocal microscope. The average velocity and its standard deviation was calculated for two measured flow rates along with their expected velocities.

Table III. Translume pressure-driven results summary. The mean and median velocity for each type of syringe pump was calculated for each flow rate to compare with the theoretical velocity.....

Table IV. Cidra pressure-driven results summary. The mean and median velocity for each type of syringe pump was calculated for each flow rate to compare with the theoretical velocity.

Table V. LabSmith Topas pressure-driven results summary. The mean and median velocity for each type of syringe pump was calculated for each flow rate to compare with the theoretical velocity.

Table VI. LabSmith PMMA pressure-driven results summary. The mean and median velocity for each type of syringe pump was calculated for each flow rate to compare with the theoretical velocity.

Table VII. Translume electro-kinetic driven results summary. The mean and median velocity for each flow rate. 


\section{LIST OF FIGURES}

Figure 1. Diffusion in a passive micro-mixer.................................................. 5

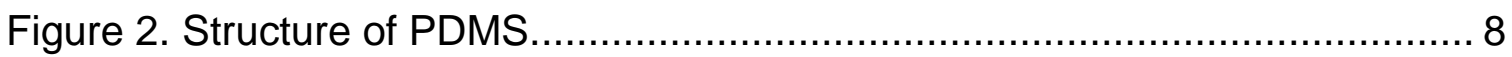

Figure 3. Processing of a PDMS microfluidic chip........................................ 9

Figure 4. A) Unit cell and B) amorphous structure of glass. ............................. 10

Figure 5. Polar fluid in a hydrophobic channel............................................ 13

Figure 6. Drop velocities for solvents under capillary force. Larger temperature differentials cause larger drop velocities. This graph shows the different drop velocities for different solvents in a $32 \times 500 \mu m$ channel. .................................................................. 14

Figure 7. Streaming potential in a channel. ................................................. 16

Figure 8. Composition of electric double layer. A layer of immobile ions and a layer of mobile ions come together to form the electric double layer. . 18

Figure 9. Electro-osmotic flow in a channel. .............................................. 20

Figure 10. Set-up of a laser doppler velocimeter............................................ 21

Figure 11. Mask designs used to fabricate channels. A) Mask used for fabrication of pressure-driven channels. The top channel was used with dimensions of $1 \mathrm{~mm}$ wide and $25 \mathrm{~mm}$ long. B) Mask designed in AutoCAD for fabrication of electro-kinetic channels. Channel dimensions are $170 \mu \mathrm{m}$ wide and $25 \mathrm{~mm}$ long. The wells are $5 \mathrm{~mm}$ squares.

Figure 12. Process flow chart for the fabrication of PDMS channels................. 25

Figure 13. Setup of pressure-driven flow channel. ...................................... 27

Figure 14. Setup of electro-kinetic flow channel. ........................................... 28

Figure 15. PIV image analysis. Example overlay of 8 images in different colors to allow for PIV measurements.

Figure 16. Color coder. Time-lapse color coder used to analyze stacks of 8 images that had been overlaid in different colors.

Figure 17. Graph of pressure-driven results. Pressure-driven results for $0.5 \mu \mathrm{L} / \mathrm{min}$ (blue) and $0.75 \mu \mathrm{L} / \mathrm{min}$ (red). For a wide and flat channel 
the parabolic profile would only be apparent near the wall of the channel (black).

Figure 18. Drawing of pressure driven PDMS channel.................................... 31

Figure 19. Setup for syringe pump verification. ………................................ 32

Figure 20. Results of syringe pump verification............................................. 33

Figure 21. Bubble in channel. Bubble traveling through the channel with $0.25 \mu \mathrm{L} / \mathrm{min}$ of pressure and $50 \mathrm{~V}$. The voltage appears to have no impact on the flow........................................................................... 34

Figure 22. Layout of Translume cross-channel............................................. 37

Figure 23. Layout of Translume straight-channel. ......................................... 37

Figure 24. Layout of Cidra single-channel flow cell. ....................................... 38

Figure 25. LabSmith PMMA optical microscope image. Optical microscope image of PMMA LabSmith cross-channel through-hole chip taken at 50x magnification. The image shows the base dimensions of the trapezoidal channel. One base has an average dimension of $100.76 \mu \mathrm{m}$ and the other base has an average dimension of $141.08 \mu \mathrm{m}$

Figure 26. Translume channel cleaning. Translume channel suspended in sonic bath to remove particles stuck to channel walls.

Figure 27. Attaching connectors. Left: Aligned connector on channel hole. Right: Epoxied connector on channel hole.

Figure 28. Effect of wetting agent on bubble in channel. Left: Channel bubble with wetting solution. Right: Channel bubble without wetting solution.

Figure 29. Pressure measurement setup. Set-up of Cidra channel with pressure-sensor system. Tubing is run from the syringe pump to a $\mathrm{T}$-connector and from the T-connector to the channel. A pressure sensor is also attached to the T-connector and to the computer to be logged. Set up is similar for other channels measured.

Figure 30. Pressure measurement for Translume channel. Output from LabSmith pressure sensor with New Era and Harvard Apparatus syringe pump. Drops during testing are from refilling the syringe....... 48

Figure 31. Pressure measurement for Cidra channel. Measurements were taken for both the New Era and Harvard Apparatus syringe pump 
using a LabSmith pressure sensor. Refilling the syringe caused drops in pressure.

Figure 32. Pressure measurement for LabSmith Topas channel. Measurements were taken by a LabSmith pressure sensor with New Era and Harvard Apparatus syringe pump. The drops in pressure are from refilling the syringe during testing.

Figure 33. Pressure measurement for LabSmith PMMA channel. Output from LabSmith pressure sensor with the New Era and Harvard Apparatus syringe pumps. The drops during testing are from refilling the syringe

Figure 34. Pressure comparison for channels with New Era syringe pump. Linear models were fit to the measured data to show the linear relationship between pressure and flow rate.

Figure 35. Harvard Apparatus syringe pump channel pressure comparisons. Linear models were fit to the measured data to show the linear relationship between pressure and flow rate.

Figure 36. Theoretical and measured pressures for Translume channel............ 54

Figure 37. Theoretical and measured pressures for Cidra channel................... 54

Figure 38. Theoretical and measured pressures for LabSmith channels............ 55

Figure 39. Representation of probe position conversion. …........................... 57

Figure 40. Setup of syringe tips and copper wire in channel inlet/outlet.............58

Figure 41. Measured voltage from LabSmith High Voltage Sequencer............. 59

Figure 42. Probe setup within Translume channel. Setup of probes within Translume channel. The probes were evenly spaced across the channel with the best size option to measure the particle velocities... 61

Figure 43. Graph for Translume channel with New Era syringe pump. 62

Figure 44. JMP output for model of Translume channel with New Era syringe pump.

Figure 45. Graph of Translume channel with Harvard Apparatus syringe pump. 64

Figure 46. JMP output for model for Translume channel with Harvard Apparatus syringe pump. 65

Figure 47. Setup of probes within Cidra channel for New Era syringe pump...... 66 
Figure 48. Graph of Cidra channel with New Era syringe pump.

Figure 49. JMP output for model for Cidra channel with New Era syringe pump.

Figure 50. Setup of probes within Cidra channel for Harvard Apparatus syringe pump.

Figure 51. Graph of Cidra channel with Harvard Apparatus syringe pump........ 70

Figure 52. JMP output for model for Cidra channel with Harvard Apparatus syringe pump.

Figure 53. Probe setup within LabSmith Topas channel. Setup of probes within LabSmith Topas channel for New Era Syringe Pump. The probes were evenly spaced across the channel with the best size option to measure the particle velocities.

Figure 54. Graph for LabSmith Topas channel with New Era syringe pump. .... 73

Figure 55. JMP output for model for LabSmith Topas channel with New Era syringe pump.

Figure 56. Probe setup within LabSmith Topas channel. Setup of probes within LabSmith Topas channel for Harvard Apparatus Syringe Pump. The probes were evenly spaced across the channel with the best size option to measure the particle velocities.

Figure 57. Graph for LabSmith Topas channel with Harvard Apparatus syringe pump.

Figure 58. JMP output for model for LabSmith Topas channel with Harvard Apparatus syringe pump.

Figure 59. Probe setup within LabSmith PMMA channel. Setup of probes within LabSmith PMMA channel. The probes were evenly spaced across the channel with the best size option to measure the particle velocities.

Figure 60. Graph of LabSmith PMMA channel with New Era syringe pump....... 79

Figure 61. JMP output for model for LabSmith PMMA channel with New Era syringe pump.

Figure 62. Graph of LabSmith PMMA channel with Harvard Apparatus syringe pump. 
Figure 63. JMP output for model for LabSmith PMMA channel with Harvard

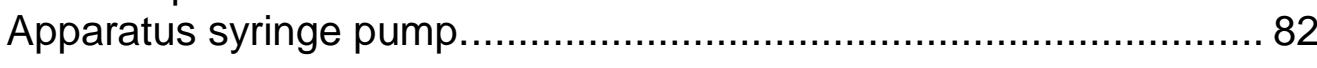

Figure 64. Model of parabolic profile in Translume channel. ............................ 84

Figure 65. Normalized model of parabolic profile. …….................................... 85

Figure 66. Measured vs. theoretical $2.5 \mu \mathrm{L} / \mathrm{min}$ Translume data. The error bars are constructed using 1 standard deviation from the mean................ 86

Figure 67. Probe setup within Translume channel. Setup of probes within Translume channel. The probes were evenly spaced across the channel with the best size option to measure the particle velocities... 89

Figure 68. Graph of Translume channel with electro-kinetic flow. .................... 90

Figure 69. JMP output for model for Translume channel with electro-kinetic flow.

Figure 70. Plot of effects of electro-osmotic and electrophoretic flow. Error bars are constructed using 1 standard error from the mean.

Figure 71. Graph of Translume channel velocity profile for pressure-driven and electro-kinetic driven flow. The $125 \mathrm{~V}$ electro-kinetic flow at about $200 \mu \mathrm{m} / \mathrm{s}$ is comparable with a $1 \mu \mathrm{L} / \mathrm{min}$ flow rate. 


\section{LIST OF EQUATIONS}

Equation 1. Reynolds number determination............................................... 11

Equation 2. Calculation of average velocity for pressure-driven flow................. 11

Equation 3. Determination of hydraulic diameter........................................... 11

Equation 4. Time to $50 \%$ mixing by diffusion............................................... 12

Equation 5. Surface tension's dependence on temperature ............................ 13

Equation 6. Determination of pressure change within channel......................... 14

Equation 7. Determination of fluid resistance ............................................. 14

Equation 8. Determination of voltage due to fluid movement. ........................... 16

Equation 9. Determination of voltage due to particle movement....................... 17

Equation 10. Particle velocity from electric field with small Debye length........... 17

Equation 11. Particle velocity from electric field with large Debye length. ......... 17

Equation 12. Calculation of Debye length...................................................... 19

Equation 13. Fluid velocity from applied electric field. .................................... 19

Equation 14. Concentration conversion between solutions. ............................. 45

Equation 15. Pressure change within micro-fluidic channel.............................53

Equation 16. Poiseuille flow in a rectangular channel....................................... 83

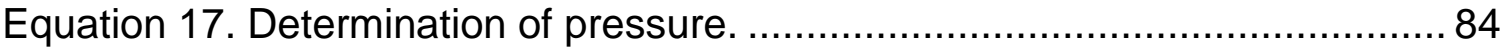




\section{ChAPTER 1 INTRODUCTION}

\subsection{DEFINITION}

Microfluidics deals with the flow of fluids, either gases or liquids, in channels with at least one dimension less than 100 micrometers. The flow in these channels differs from macro-scale fluid flows due to laminar flow at low Reynolds numbers.

There are natural channels on the micro-scale, but normally the term microfluidics refers to human-made objects. Microfluidic chips, a group of channels, can be etched or molded into a variety of materials as described in Section V. Different connections between the channels perform different functions. The channels are normally connected to the outside world through input and output holes and other connections to direct flow [1].

Microfluidics technology developed out of the semiconductor industry, so much of the processing is based on the processing of silicon. However, microfluidic channels can also be made of glass, ceramics, metals, and polymers because of more recent processing steps. Channel materials are chosen based on fluid compatibility, production time, costs, and the final application. PDMS (poly dimethyl siloxane), detailed in Section V Part A, is often used in research because of its low cost and quick processing [1].

\subsection{HISTORY}

The semiconductor industry has launched many spinoff technologies, including microfluidics. In the 1980's, silicon etching processes were developed that allowed the production of cantilever and diaphragms, also called MEMS (micro 
electromechanical systems) used in many sensory applications [2]. In the 1990's, research was done to use these systems as diagnostic tools in biology and chemistry mainly for use in hospitals, leading to the development of microfluidic systems, also called lab on a chip. This research expanded with the use of PDMS because of its low costs and quick production [1].

\subsection{PROJECT DEFINITION/RESEARCH QUESTION}

The goal of this project is to compare electro-kinetic and pressure driven flow rates and velocity profiles (near wall vs. middle) in a microfluidic chip made of PDMS and/or glass using particle imaging velocimetry (PIV) of an aqueous solution of fluorescent polystyrene (PS) particles on a laser confocal microscope (LCM).

The expectation was that flow rates will have different ranges between the two methods because of allowed pressure within a channel without leaking. The electro-kinetic system will have a more uniform velocity profile allowing more even and quicker mixing, being better for mixing applications.

Previous research at Cal Poly has involved pressure-driven flow. This research determined a method for creating electro-kinetic driven flow in Cal Poly labs. Both these flow techniques were measured for comparison.

\subsection{OVERALl APPROACH}

Solutions were made of fluorescent polystyrene particles, ranging from $1 \mu \mathrm{m}$ to $10 \mu \mathrm{m}$ in size. These solutions were pumped through a variety of channels using pressure-driven and electro-kinetic driven flows. Probes were placed over 
various regions of the channels to obtain a velocity profile. A probe is a part of UScope's software that analyzes the video. In this case, the probe determines particle movement from frame to frame and finds the velocity of the particles within the probe area. These profiles were compared to existing models. Finally, the measured flow profiles of pressure-driven and electro-kinetic driven flows were compared for the same channel. 


\section{Chapter 2 Background \& Literature RevieW}

\subsection{COMPONENTS OF A DEVICE}

Common microfluidic components include filters, needles, mixers, and separators [3]. Most electro-kinetic flow applications use mixers and separators for more even mixing and to separate charged components from a solution.

\subsubsection{Micro-Filters}

Filters separate either large or small molecules based on physical size. Filter designs consider the distribution of the filtered particles, the pressure loss due to the filtering, and the mechanical strength of the filter to prevent break down over time [4].

\subsubsection{Micro-Needles}

Mainly used in the medical industry to deliver medication, micro-needles are a painless way of administering drugs. Designs consider the strength of the needles, the material's biocompatibility, and the flow of the medication through the needle [5].

\subsubsection{Micro-Mixers}

There are two types of micro-mixers: those with moving parts, active micromixers, and those without, passive micro-mixers. In both types, mixing is controlled by diffusion, which can be modeled by Fick's first and second laws (Figure 1) [1]. Fick's first law relates diffusion rate and concentration while Fick's second law relates concentration and time. Bends and obstacles can be added to a path to assist in mixing for passive micro-mixers. T-mixers and Y-mixers are common examples of passive micro-mixers. To increase passive mixing, multiple 
streams can be put into a passive mixer to increase the surface area over which diffusion occurs. Active micro-mixers utilize pressure, voltage potentials and micro-stirrers to assist in mixing [5].

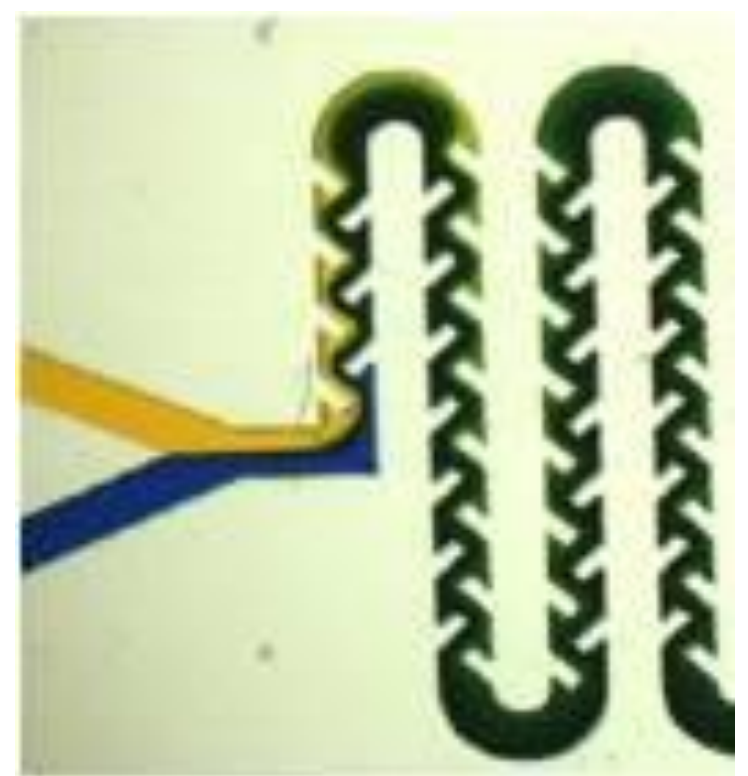

Figure 1. Diffusion in a passive micro-mixer.

\subsubsection{Micro-Separators}

Separators isolate particles based on unique properties. Particles can be separated based on properties other than size, like electrical and magnetic properties [4].

\subsection{APPLICATIONS}

Researchers from many different fields of engineering and science are actively pursuing topics in microfluidics. The five major commercial applications of microfluidics are medical diagnostics, genetic sequencing, chemistry production, drug discovery, and proteomics [3]. 


\subsubsection{Medical Diagnostics}

Microfluidic devices are being developed to detect viruses and bacteria. These detection devices require smaller samples, less output time, and less lab work than traditional diagnostic approaches, getting treatments to people faster [6]. In addition, multiple tests can be run simultaneously on the same device.

\subsubsection{Genetic Sequencing}

DNA sequencing on the macro-scale requires amplification, a process that copies the DNA strands by a multi-step process that denatures, anneals, and extends. Denaturing splits the double DNA strands into single strands of DNA. Annealing and extension create the complimentary strands. The DNA strands are then separated and sequenced using various techniques. On the micro-scale, the main benefits are less reagent costs due to smaller volumes, quicker heating, quicker reaction times, laminar flow allowing the use of electro-osmotic or capillary flow, and precisely defined volumes. However, possible problems include samples degrading or evaporating [7].

\subsubsection{Chemistry Production}

Chemist and chemical engineers work to create industry scale reactions to create products from available materials. Currently, processes involve scaling-up which reduces precision and can be expensive. Microfluidics will allow for new reaction mechanisms. In addition, putting multiple chips in a sequence allows for interchangeable pieces creating customizable reactions from pre-manufactured items [8]. 


\subsubsection{Drug Discovery}

Drugs are being developed all the time for different ailments; however, about a decade of testing is required before reaching the market. Through microfluidics, the new drugs can be analyzed more precisely and quickly using smaller amounts of reagents. However, these devices need to be versatile for multiple drug types and multiple testing situations. These devices can also be used to determine the correct dosage [9].

\subsubsection{Proteomics}

Proteomics is the study of proteins, which involves identifying the protein (profile), determining the purpose of the protein (function), and determining how the protein folds (structure). Microfluidic devices are mainly used in the profile and function of proteomics. This analysis usually requires multiple repeating steps and large amounts of costly reagents. Microfluidics would be able to perform these steps quickly with much less reagent [10].

\subsection{MATERIALS IN USE}

The numerous materials that can be used to make microfluidic channels include glass, metals, ceramics, and polymers. This report focuses on PDMS because of its ease of processing, flexibility, and transparency; and glass because of its ability to be plasma bonded to PDMS and its ease of use with a laser confocal microscope. 


\subsubsection{PDMS}

PDMS (poly dimethyl siloxane) is a silicon based elastomer that comes as a base and a curing agent, from its supplier Dow Corning, which are mixed in a 10:1 ratio (Figure 2) [1].

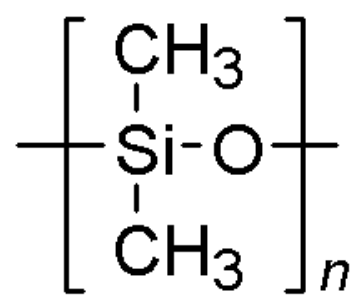

Figure 2. Structure of PDMS.

The processing can easily be completed in about 2 hours with a premade mold [11]. The base contains monomer, while the curing agent contains a cross-linker. The base is a viscous liquid, but after cross-linking becomes a hydrophobic flexible solid. Treatments can be done to make the surface temporarily hydrophilic. Plasma oxidizes the surface replacing methyl groups with hydroxyl groups. Plasma can also be used to bond the PDMS surface to another surface like glass or another piece of PDMS, which is useful to make a fourth wall of a microfluidic channel [1].

There are many advantages to using PDMS in microfluidics research. PDMS is inexpensive and easily processed with good resolution. In addition, PDMS creates a strong bond to glass or another PDMS layer with plasma bonding. The thickness of a PDMS layer can be controlled with a spin coating step. PDMS is flexible, so input tubes can be easily integrated into a device. Biocompatibility is also a very desirable property for many research applications [1]. 
There are also issues with using PDMS in microfluidic systems. Electro-kinetic flow is problematic with PDMS because depositing metals on PDMS is difficult; however, metals can be deposited on glass which is then plasma bonded to the PDMS. In addition, PDMS ages over time, which might change desired mechanical properties. PDMS also dissolves in many organic solvents which prevents some solvents being used for certain reactions [1].

PDMS processing is very simple (Figure 3) [1]. First, a mold is made using soft lithography on SU-8, a common negative photoresist. The base and curing agent is mixed and poured over the mold. Bubbles normally need to be removed using a vacuum chamber. After the PDMS has set, the mold is released and inlet and outlet holes are made in the PDMS. The PDMS is then plasma treated and attached to another surface. Connectors can then be inserted and the device can be used [1].

(1)

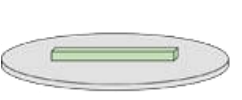

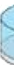

Master mold

(4)

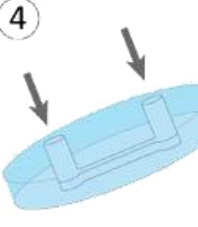

Inlet drilling
2

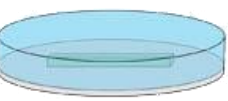

PDMS pouring $\&$ reticulation

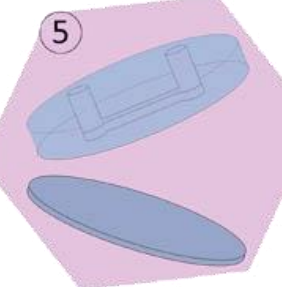

Plasma treatment
3

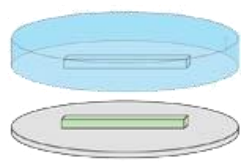

Release of PDMS replica

6

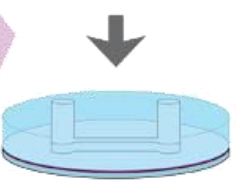

Put in contact for bonding

Figure 3. Processing of a PDMS microfluidic chip. 


\subsubsection{Glass}

Glass is made of silicon and oxygen arranged in an amorphous structure (Figure 4) [12]. Like PDMS, glass can be plasma bonded. The bonding of PDMS to glass is slightly weaker than the bonding of PDMS to PDMS, which must be considered when determining the pressure within a channel. However, bonding to a slide or cover-slide could make viewing under a microscope easier.

A

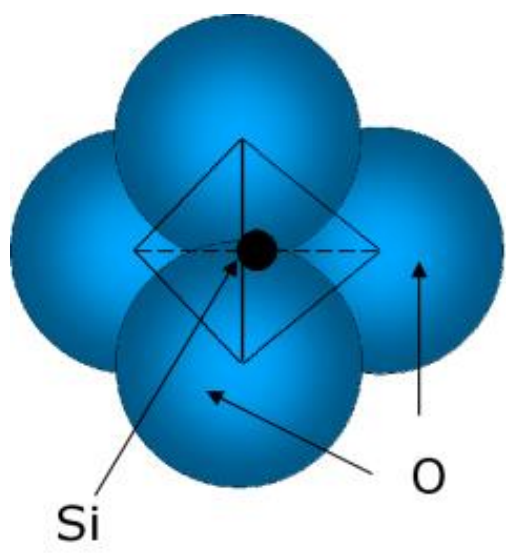

Figure 4. A) Unit cell and B) amorphous structure of glass.

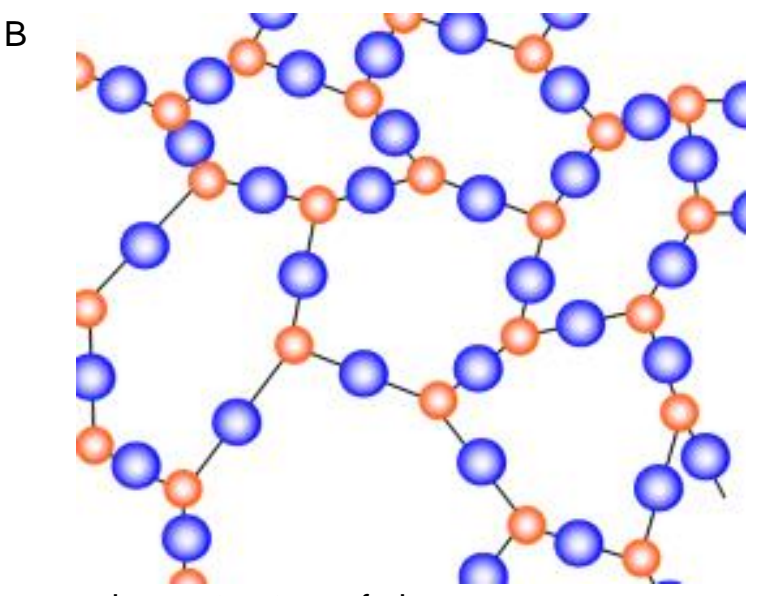

B

\subsubsection{Other Polymers}

PMMA, or poly(methyl methacrylate), also known as Plexiglas, is often used in hard contact lenses because of its optical clarity [13].

TOPAS is the commercial name for a group of cyclic olefin copolymers. These polymers are amorphous with high optical transparency and low water absorption. TOPAS is chemically resistant to IPA, acetone, methanol, and sulfuric acid. There is no information on TOPAS reactivity with potassium hydroxide, but in general it should be resistant to aqueous acids and bases [14]. There may be some auto-fluorescence that might cause issues when imaging [15]. 


\subsection{METHODS OF FLOW}

\subsubsection{Determining Type of Flow}

On the micro-scale, the type of flow must be considered in the channel. Turbulent flow is what is normally seen on the macro-scale; however, laminar flow is often seen on the micro-scale. Since microfluidics involves making devices on the micro-scale, understanding laminar flow is important. The Reynolds number can determine what kind of flow will be present [16]:

Equation 1. Reynolds number determination.

$$
R_{e}=\frac{\rho V D}{\mu_{d}}
$$

Where $\rho$ is the density (water $=1 \mathrm{~g} / \mathrm{cm}^{3}$ ), $V$ is the velocity, $D$ is the hydraulic diameter, and $\mu_{d}$ is the dynamic viscosity (water $=1 * 10^{-3} \mathrm{~kg} / \mathrm{m}^{*} \mathrm{~s}$ ). For liquids, if the Reynolds number is less than 2000 , the fluid is expected to exhibit laminar flow. $V$ can be calculated by:

Equation 2. Calculation of average velocity for pressure-driven flow.

$$
V=\frac{Q}{A}
$$

Where $Q$ is the flow rate and $A$ is the cross-sectional area. $D$, for a rectangular channel, can be calculated by:

Equation 3. Determination of hydraulic diameter.

$$
D=\frac{2 a b}{(a+b)}
$$

Where $a$ and $b$ are the lengths of the sides. For liquids, laminar flow is present for Reynolds numbers below 2000. 
For laminar flow, where mixing depends on diffusion, mixing time can be estimated by:

Equation 4 . Time to $50 \%$ mixing by diffusion.

$$
t_{50 \%}=\frac{w^{2}}{D}
$$

Where $w$ is the width of the channel and $D$ is the diffusivity (water $=1^{*} 10^{-9} \mathrm{~m}^{2} / \mathrm{s}$ ).

As discussed earlier, micro-mixers are one of the many uses of microfluidics. The time and flow rate are used to determine a mixing length. The above equations estimate the amount of mixing that occurs within a channel of a specified length. This length is used when designing devices.

Although diffusion is a major part of mixing on this scale, other factors also contribute to the degree of mixing, such as velocity profile. How different methods of inducing flow create different types of velocity profiles is discussed in the following sections.

\subsubsection{Flow Driven by Capillary Forces}

In nature, capillary forces are used to move liquid up small tube-like structures without any additional forces. This process requires the tubes surface to be hydrophilic, unlike PDMS. Adding additional forces can make water flow through a PDMS channel. Capillary pumping applies a temperature gradient across liquid in a channel. The difference in surface tension because of the temperature gradient causes liquid to move through the channel (Figure 5) [17]. A drop will have equal pressure, or surface tension, on both sides and not move, but adding 
a temperature gradient changes the pressure and allows the liquid to move toward the higher temperature.

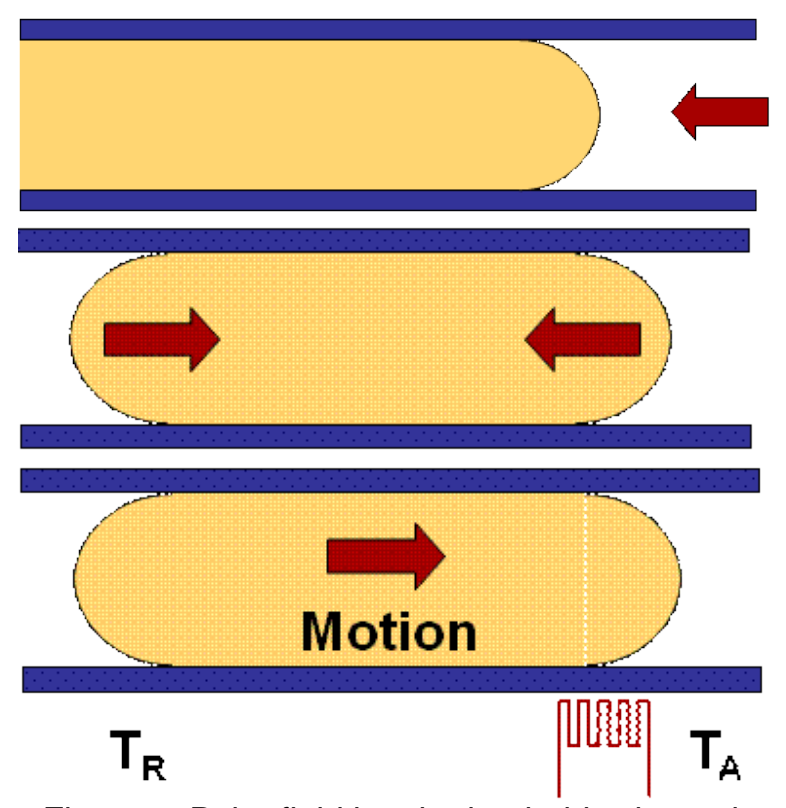

Figure 5. Polar fluid in a hydrophobic channel.

Surface tension can be calculated by:

Equation 5. Surface tension's dependence on temperature.

$$
\gamma=a-b T
$$

Where $\gamma$ is surface tension, $a$ is $75.83 \mathrm{dyn} / \mathrm{cm}$ and $b$ is $0.1477 \mathrm{dyn} / \mathrm{cm} /{ }^{\circ} \mathrm{C}$ for water, and $T$ is temperature. As shown in the equation, surface tension is dependent on temperature. Knowing how temperature affects surface tension will allow predictions about the direction of flow. The change in temperature across the drop determines how fast the drop moves through the channel, with higher temperature changes resulting in higher drop velocities (Figure 6) [17]. 


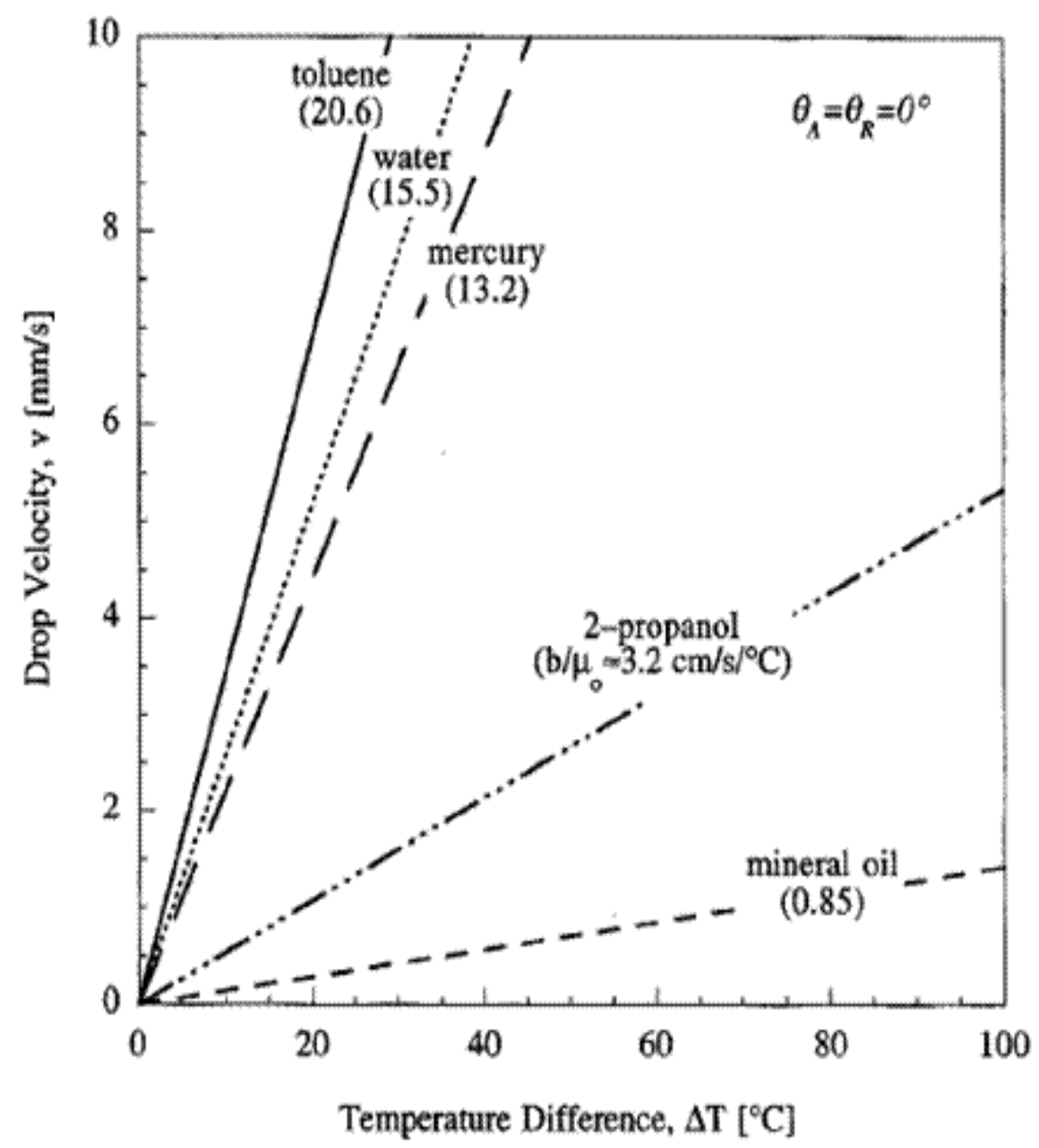

Figure 6. Drop velocities for solvents under capillary force. Larger temperature differentials cause larger drop velocities. This graph shows the different drop velocities for different solvents in a $32 \times 500 \mu \mathrm{m}$ channel.

\subsubsection{Pressure Driven Flow}

Pressure driven flow is related to the flow rate and fluid resistance by:

Equation 6. Determination of pressure change within channel.

$$
\Delta P=Q R
$$

Where $\Delta P$ is the change in pressure within the channel, $Q$ is the flow rate, and $R$ is the fluid flow. Fluid flow is calculated by:

Equation 7. Determination of fluid resistance.

$$
R=\frac{12 \mu_{d} L}{w h^{3}}
$$


Where $\mu_{d}$ is the dynamic viscosity (water $=1 * 10^{-3} \mathrm{~kg} / \mathrm{m}^{*} 2$ ), and $L, w$, and $h$ are the dimensions of the channel. The velocity can be calculated by Equation 2 . Often a syringe pump is used to apply a specific flow rate.

The pressure needs to be low enough to not break the bonding of the PDMS to the glass cover slide or leak at the input and output holes. The velocity of the fluid must be within a range so that a single particle within the fluid remains within the frame of view of the microscope. More detail about the microscope is described in Section VII. In addition, pressure within the channel and the fluid's velocity increases with increasing applied flow rate.

Pressure driven flow is known for a parabolic profile when moving liquid through the channel. This means that the fluid flows at different rates near the wall of the channel than at the middle.

\subsubsection{Electro-Kinetic Forces}

Although there are many different types of electro-kinetic phenomena, four main types are of interest (Table I). These forces differ in whether a force or an electric field is applied and whether that application causes a solid or liquid to move.

Table I. Electro-kinetic force comparison chart. The four main types of electro-kinetic forces can be categorized by the movement of solids and liquids in the system and what is applied to the system.

\begin{tabular}{|c|c|c|}
\hline & $\begin{array}{l}\text { Stationary } \\
\text { Solid }\end{array}$ & $\begin{array}{l}\text { Stationary } \\
\text { Liquid }\end{array}$ \\
\hline $\begin{array}{l}\text { Apply } \\
\text { Force }\end{array}$ & $\begin{array}{l}\text { Streaming } \\
\text { Potential }\end{array}$ & $\begin{array}{c}\text { Sedimentation } \\
\text { Potential }\end{array}$ \\
\hline $\begin{array}{c}\text { Apply } \\
\text { Electric Field }\end{array}$ & Electro-Osmosis & Electrophoresis \\
\hline
\end{tabular}




\section{Streaming Potential}

Streaming potential occurs when an electric field is created by the movement of a polar liquid. This happens with pressure driven flow through a channel. The created potential can be calculated by:

Equation 8. Determination of voltage due to fluid movement.

$$
V=\frac{\zeta \varepsilon_{0} \varepsilon_{r} \Delta P}{\eta \kappa}
$$

Where $\zeta$ is the zeta potential of the wall and liquid interface, $\varepsilon_{o}$ is the permittivity of free space $\left(8.85 \times 10^{-21} \mathrm{As} / \mathrm{Vm}\right), \varepsilon_{r}$ is the relative permittivity for PDMS, $\Delta P$ is the applied pressure, $\eta$ is the viscosity and $\kappa$ is the solution's conductivity [18].

Streaming potential also has a parabolic profile due to the difference in ion concentrations near the wall and at the middle of the channel (Figure 7) [19].

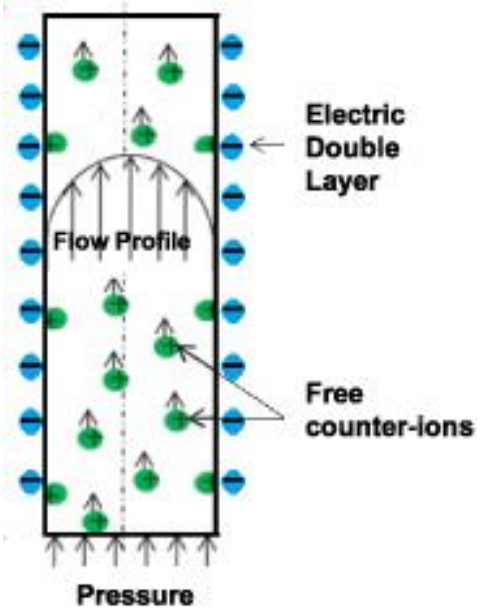

Figure 7. Streaming potential in a channel. 


\section{Sedimentary Potential}

Sedimentary potential occurs when an electric field is created by the movement of particles, often particles settling in a liquid. The created potential can be calculated by:

Equation 9. Determination of voltage due to particle movement.

$$
V=\frac{\zeta \varepsilon_{0} \varepsilon_{r}\left(\rho-\rho_{o}\right) g}{\eta \kappa}
$$

Where $\zeta$ is the zeta potential of the wall and liquid interface, $\varepsilon_{o}$ is the permittivity of free space $\left(8.85 \times 10^{-21} \mathrm{As} / \mathrm{Vm}\right), \varepsilon_{r}$ is the relative permittivity for PDMS, $\rho$ is the density of the solution, $\rho_{o}$ is the density of the solvent, $g$ is the force of gravity $\left(9.81 \mathrm{~m} / \mathrm{s}^{2}\right), \eta$ is the viscosity and $k$ is the solution's conductivity [18]. Streaming and sedimentary potential can occur together and counteract each other.

\section{Electrophoresis}

Electrophoresis occurs when particles move due to an applied voltage, due to surface charges. The velocity of the particles can be calculated by

If the Debye length, a measure of electrostatic affect in a solution, is small compared to the particle radius:

Equation 10. Particle velocity from electric field with small Debye length.

$$
v=\frac{\varepsilon_{0} \varepsilon_{r} \zeta \vec{E}}{\eta}
$$

If the Debye length is large compared to the particle radius,

Equation 11. Particle velocity from electric field with large Debye length. 


$$
v=\frac{2}{3} \frac{\varepsilon_{0} \varepsilon_{r} \zeta \vec{E}}{\eta}
$$

Where $\varepsilon_{0}$ is the permittivity of free space $\left(8.85 \times 10^{-12} \mathrm{As} / \mathrm{Vm}\right), \varepsilon_{r}$ is the relative permittivity for PDMS, $\zeta$ is the zeta potential for the wall liquid interface, $\vec{E}$ is the applied electric field, and $\eta$ is the viscosity [18].

The Debye length is a measure of ions near a surface. All surfaces will have some charge which will attract ions of opposite charge in the liquid. There will be a layer of immobile ions called the Stern layer and a layer of mobile ions called the diffuse layer. Together these are called the electric double layer (Figure 8) [20].

No net charge far from wall
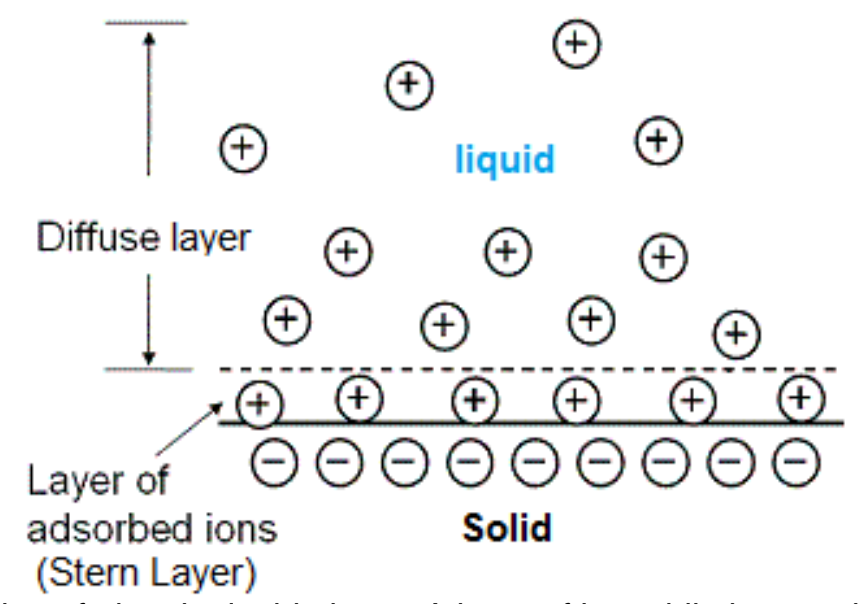

Figure 8. Composition of electric double layer. A layer of immobile ions and a layer of mobile ions come together to form the electric double layer.

The Debye length can be calculated by: 
Equation 12. Calculation of Debye length.

$$
\lambda_{D}=\frac{1}{\kappa}=\sqrt{\frac{\epsilon_{o} \epsilon_{r} k_{b} T}{e^{2} \sum n_{i} z_{i}^{2}}}
$$

Where $\lambda_{D}$ is the Debye length, $\varepsilon_{0}$ is the permittivity of free space $\left(8.85 \times 10^{-}\right.$ ${ }^{12} \mathrm{C}^{2} / \mathrm{Jm}$ ), $\varepsilon_{r}$ is the relative permittivity (76 for water), $k_{b}$ is Boltzman's constant $\left(1.38 \times 10^{-23} \mathrm{~J} / \mathrm{K}\right), T$ is the temperature, $e$ is the charge of an electron $\left(1.602 \times 10^{-}\right.$ $\left.{ }^{19} \mathrm{C}\right), n_{i}$ is the number of ion type $\mathrm{i}$, and $z_{i}$ is the charge or ion type $\mathrm{i}$. For a $\mathrm{pH}$ 7.00 Buffer with $0.021 \mathrm{M}$ of potassium dihydrogen phosphate and $0.029 \mathrm{M}$ of disodium hydrogen phosphate, the Debye length was calculated to be $913.67 \mathrm{pm}$.

\section{Electro-Osmosis}

Electro-osmosis occurs when fluid moves due to an applied voltage (Figure 9) [21]. The velocity of the fluid can be calculated by:

Equation 13. Fluid velocity from applied electric field.

$$
v=\frac{-\varepsilon_{0} \varepsilon_{r} \zeta \vec{E}}{\eta}
$$

Where $\varepsilon_{0}$ is the permittivity of free space $\left(8.85 \times 10^{-12} \mathrm{As} / \mathrm{Vm}\right), \varepsilon_{r}$ is the relative permittivity for PDMS, $\zeta$ is the zeta potential for the wall liquid interface, $\vec{E}$ is the applied electric field, and $\eta$ is the viscosity. Electro-osmosis has a plug-like profile, making more uniformity across the channel. A uniform profile is ideal in mixing systems, creating a more uniform mix more quickly. 


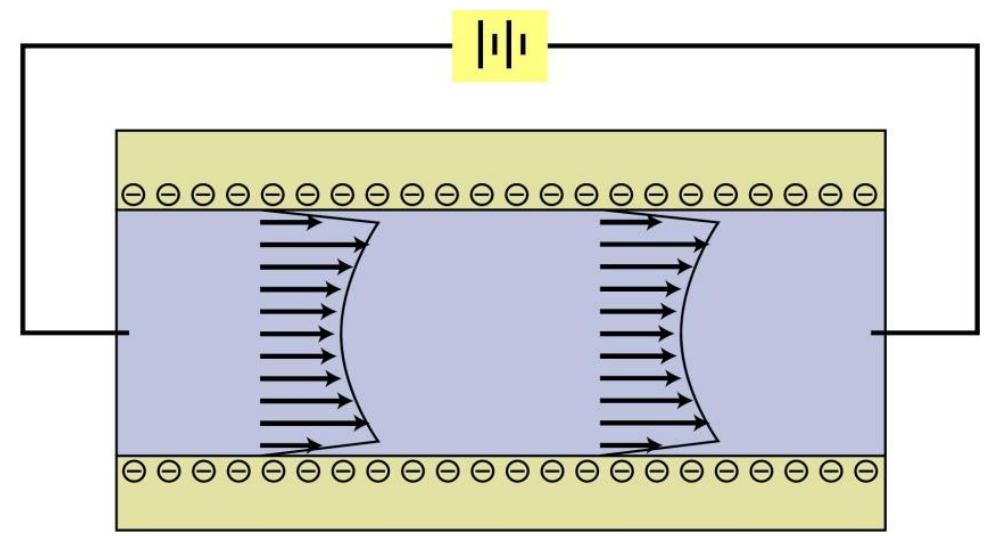

Figure 9. Electro-osmotic flow in a channel.

Since fluid cannot be seen flowing under a microscope, particles are often added. If the particles have a surface charge, they are acted on by electrophoresis in addition to the pressure of electro-osmosis from the fluid. This could cause different results than expected [18]. Electro-osmosis is the goal when applying an electric field on a microfluidic chip; however, if the particles in the fluid have a surface charge, then an electrophoretic force also acts on them. Overall, the sum of the electro-osmotic and electrophoretic forces is measured.

\subsection{MEASURING VELOCITY}

Knowing the velocity of liquid within a channel is important when designing microfluidic devices so that the correct type of flow is used for the specific application. The different ways to measure fluid velocity using flowmeters include:

- Differential Pressure Flowmeters

- Velocity Flowmeters

- Positive Displacement Flowmeters

- Mass Flowmeters

- Open Channel Flowmeters [22] 
However, these flowmeters are meant to be used on the macro-scale and are not sensitive enough for the micro-scale.

For micro-fluidics other flow measurement techniques are used such as laser doppler velocimetry, molecular tagging velocimetry, and particle image velocimetry [3].

\subsubsection{Laser Doppler Velocimetry (LDV)}

Like the Doppler effect that is heard with moving objects, this method uses the Doppler effect with light. Particles are added to a fluid that scatters a particular wavelength of light. A monochromatic laser is used to provide the light source which is then detected by a photomultiplier tube (PMT). The difference in wavelengths can then be used to determine the speed of the particles in the fluid (Figure 10) [23]. This type of measurement does not require contact with the channel and can get measurements quickly, but requires the channel to be made of transparent materials, have varying accuracy, and are expensive [23].

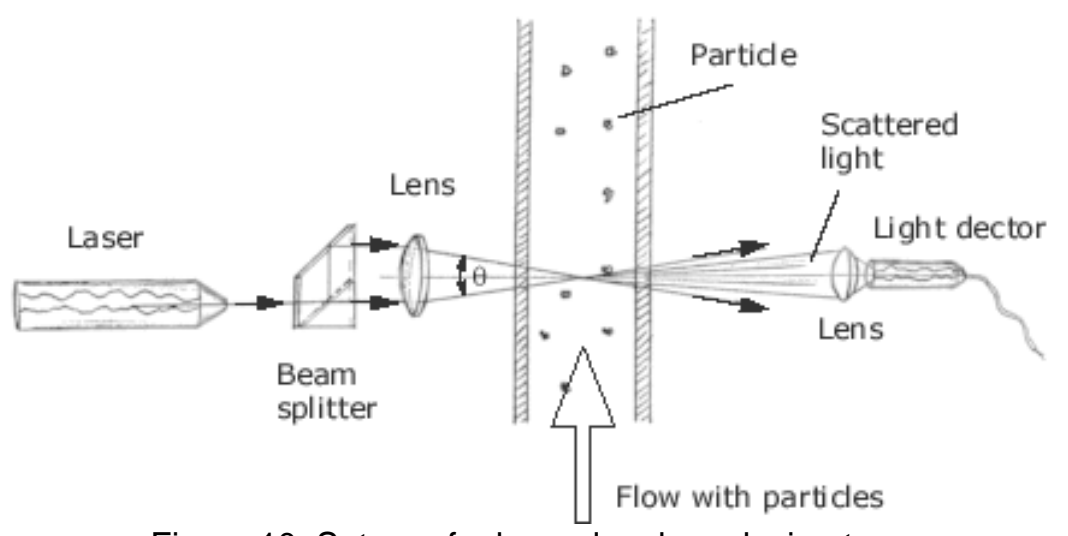

Figure 10. Set-up of a laser doppler velocimeter. 


\subsubsection{Molecular Tagging Velocimetry (MTV)}

For MTV, fluorescent or phosphorescent molecules are added to a fluid. A light source is used to excite the molecules, normally in a pattern like a grid. These are imaged multiple times in a short time interval and then processed to create velocity measurements [24].

\subsubsection{Particle Image Velocimetry (PIV)}

Like MTV, PIV uses fluorescence to see fluid flow. Fluorescent particles are added to the fluid and images are taken in short time intervals. These are then processed to create velocity measurements [3].

In this case, fluorescent polystyrene (PS) particles are used. PS particles of a specific size or molecular weight are easy to manufacture since the polymerization process is easily controlled. Fluorescent molecules can be attached to these polymer particles. Fluorescence is the absorption of high energy light and then emitting lower energy light.

A laser confocal microscope can be used to view these particles [25]. This microscope can change the light used to view only the fluorescent particles. In addition, the microscope uses a dichromatic mirror to only see emitted light and not reflected excitation light. Also optical lenses are used to separate the focal point of different colors of light. A pinhole can be put at this focal point to filter out the undesirable colors. A laser is used as the excitation light. There is a small depth of field on this type of microscope, so only a certain plane in the sample is seen at one time. 
Some microscopes are specifically made for PIV on micro-fluidic devices, like the LabSmith Synchronized Video Microscope [26]. This microscope has a wide stage and a variety of magnifications ideal for microfluidic chips. The live video can be analyzed using the LabSmith computer software. Probes of varying sizes and properties can be placed throughout the channel to attain the desired measurements.

\subsection{SOCIETAL IMPACT}

The field of microfluidics contributes to society in many positive ways. In the medical field, microfluidics is used to supply a steadier concentration of medication to patients to eliminate dangerous spikes and valleys. This is especially helpful for insulin and blood pressure medicines. Microfluidic devices allow medication to be administered even when trained personnel are not available, broadening the ability to improve health world-wide. In addition, there are environmental benefits. Waste from medical research and products, such as needles, is extensive. Microfluidics uses less material, creating less waste, and, therefore, reducing the amount trash headed to our landfills [20]. 


\section{CHAPTER 3 FOUNDATIONAL EXPERIMENTS}

\subsection{EXPERIMENTAL PROCEDURES}

A mask from a previous senior project was used to create the pressure-driven channels. While testing these channels, parameters for the laser confocal microscope were utilized. A new mask was fabricated in AutoCAD for the electrokinetic channels with these parameters (Figure 11).

A

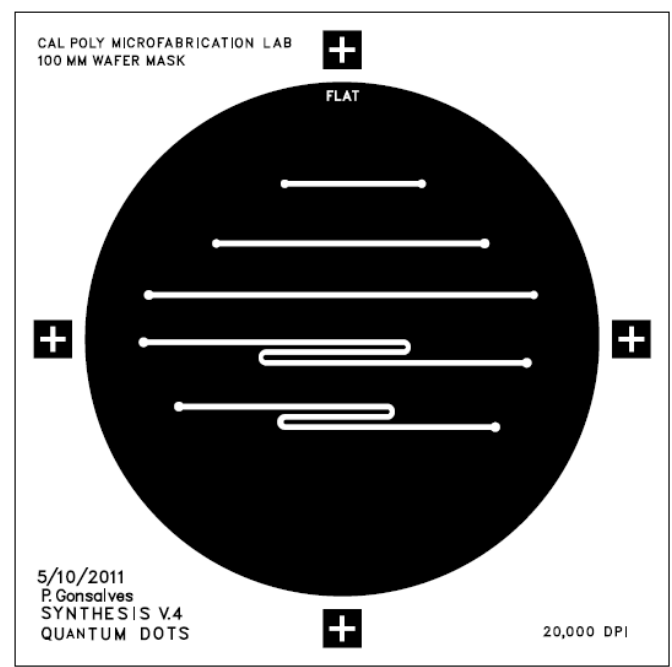

B

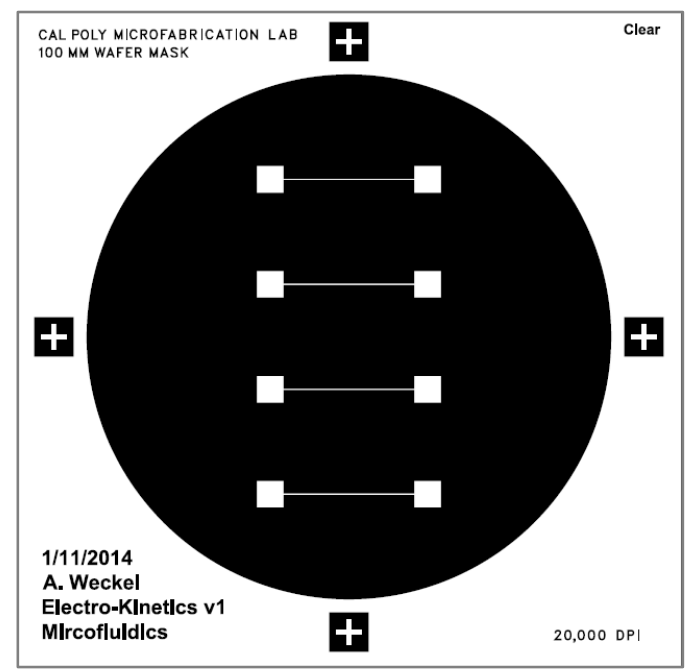

Figure 11. Mask designs used to fabricate channels. A) Mask used for fabrication of pressuredriven channels. The top channel was used with dimensions of $1 \mathrm{~mm}$ wide and $25 \mathrm{~mm}$ long. B) Mask designed in AutoCAD for fabrication of electro-kinetic channels. Channel dimensions are $170 \mu \mathrm{m}$ wide and $25 \mathrm{~mm}$ long. The wells are $5 \mathrm{~mm}$ squares.

\subsubsection{Fabrication}

Microfluidic channels were made using soft lithography processing methods (Figure 12). 

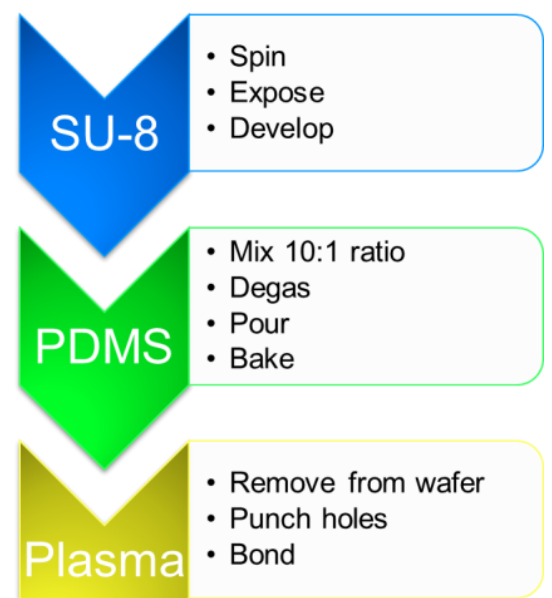

Figure 12. Process flow chart for the fabrication of PDMS channels.

A mold was made using SU-8 on a silicon wafer. The SU-8 was spun so that the pressure driven channels were $180 \mu \mathrm{m}$ deep and the electro-kinetic channels were $45 \mu \mathrm{m}$ deep.

Because the microscope is inverted, with the stage above the objective, compared to traditional microscopes, with the stage below the objective, about $100 \mathrm{~mL}$ of PDMS mixture was poured over the wafers to create a thick channel to give some support. One hole was punched at each end for the pressure-driven channel and two holes were punched for the electro-kinetic channel, one for the tubing and one for the wire.

First, the individual channels were cut out and a cover-slide was cleaned. They were placed on an acrylic surface and treated with plasma about 5 times, or about 30 to 45 seconds, and then quickly placed together. Pressure was carefully added around the channel without putting pressure on the channel to avoid bonding within the channel. 
At first the PDMS channels were directly bonded to a glass cover-slide. This caused some issues during testing with many particles sticking to the walls which made following a particle across the channel difficult. For the final channels, a thin layer of PDMS was spun on the cover-slide prior to plasma bonding. This helped a little with reducing the amount of particles sticking, but very few particles seemed to be moving. Using the same diluted solution on the sticking channels may have caused the solution to be diluted further, so a dilution of about 3.4 million beads $/ \mathrm{mL}$ was remixed from the original particle solution of $2 \%$ $2 \mu \mathrm{m}$ fluorescent carboxylate modified polystyrene beads.

\subsubsection{Testing}

\section{Microscope Parameters}

Twenty mil inner diameter tubing was used to insert fluid into the channel. Images were taken at $10 x$ magnification. The images were 256 pixels square. Each pixel was taken $2 \mu$ s apart going across and down the image. The pixel size was $4.971 \mu \mathrm{m}$ square.

The channel was set up on the laser confocal microscope, which is an inverted microscope. Fluid was pumped throughout the channel and tubing. This fluid was then allowed to come to rest which normally took about 30 minutes.

\section{Pressure-Driven Flow Setup}

For pressure-driven flow, a New Era Pump Systems syringe pump was used (Figure 13). A $1 \mathrm{~mL}$ syringe pump was used rather than a $3 \mathrm{~mL}$ syringe pump with 
an inner diameter of $4.69 \mathrm{~mm}$. These syringe pumps have a tendency to pulse at lower flow rates, which may cause inconsistent measurements.

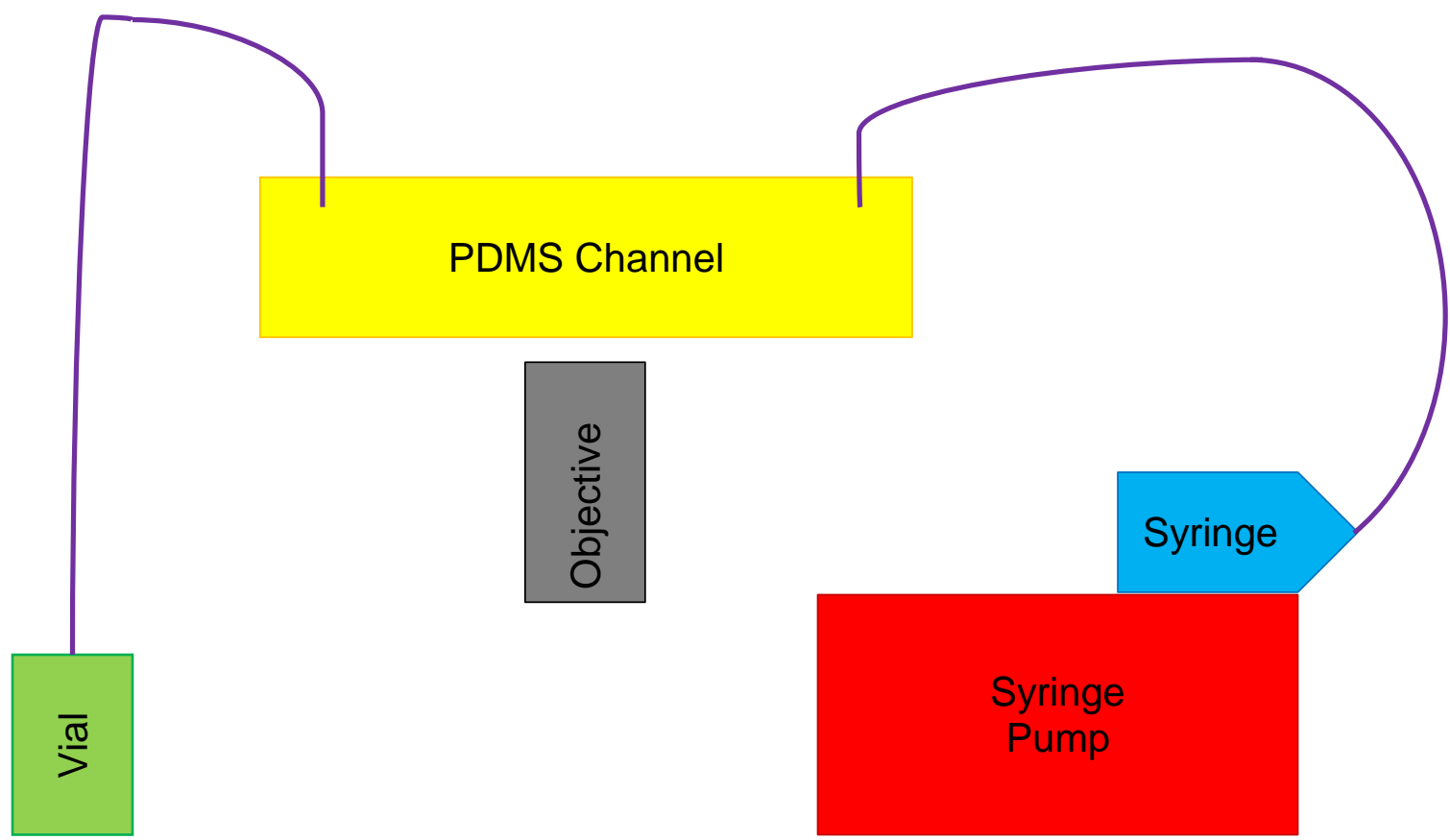

Figure 13. Setup of pressure-driven flow channel.

For pressure-driven flow a pump rate was set on the syringe pump and allowed to pump for 10 minutes before images were taken.

\section{Electro-Kinetic Flow Setup}

Multiple methods were used to obtain data for electro-kinetic flow, but none produced consistent results (Figure 14). At first, a voltage was applied along the channel with electrodes placed $25 \mathrm{~mm}$ apart, which had varied results. The particles would flow in one direction for a while and then switch directions. Some force build up was hypothesized to be causing this switch. A small flow rate was applied as a base in addition to the applied voltage to try to remove the effects of the force build up, but the flow rate seemed to overpower the voltage. 


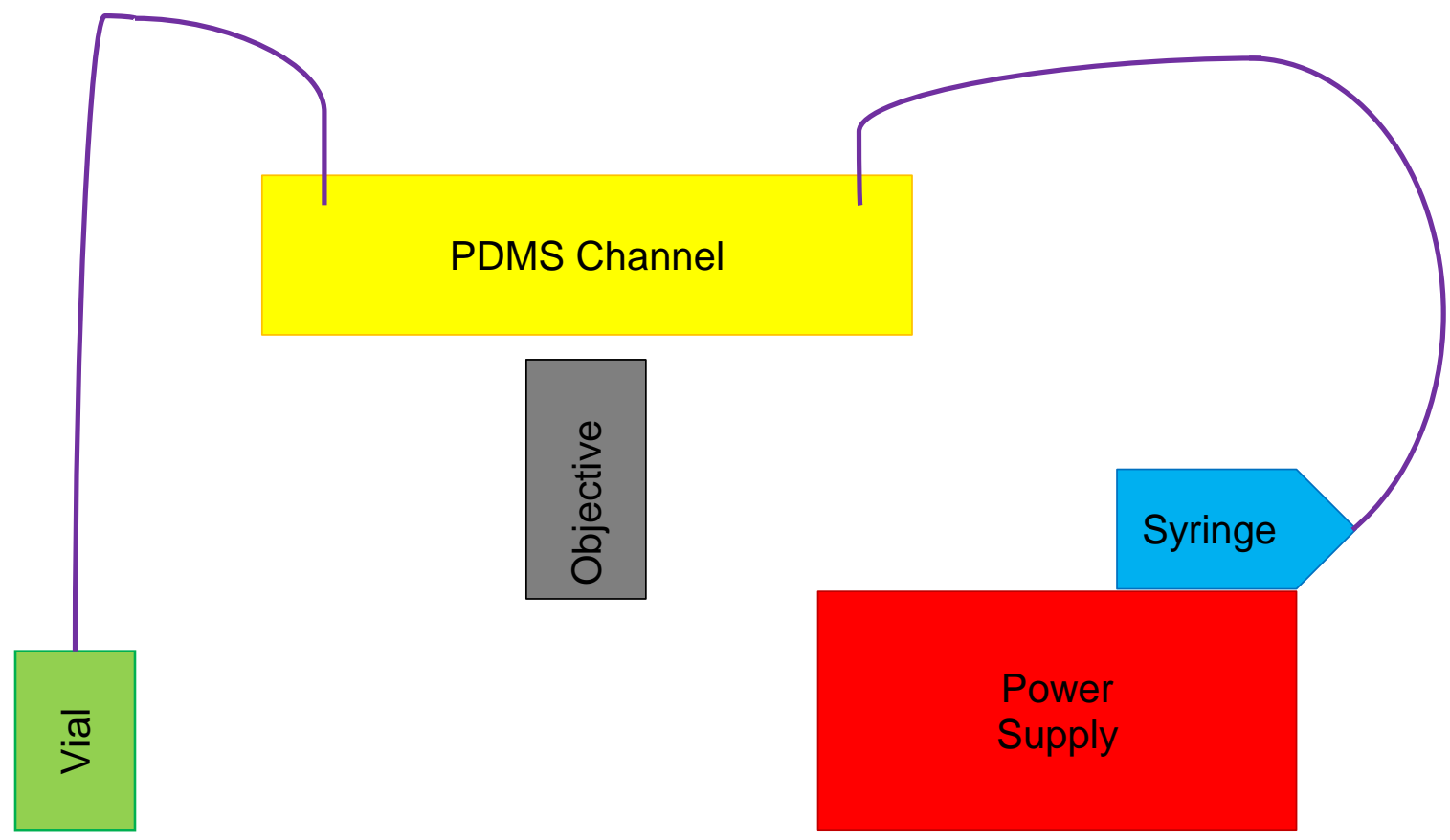

Figure 14. Setup of electro-kinetic flow channel.

Many difficulties were found during testing. Measurements were taken that yielded values close to the calculated value, much higher, and much lower. Air bubbles in the system interfered with the particle movement, causing very strange numbers. Saline solutions had different surface properties from DI water which is very influential for the electro-kinetics, but after a while the particles would stop moving.

\subsection{ANALYSIS}

The images were taken at one plane in the channel at multiple times. The microscope camera was set to take multiple-tiff images in stacks of 50 . Substacks were made and overlaid in different colors using Image-J software. Originally, only 2 images were used for the overlay, but measurements might not be the same particle at two different times but two different particles going in and out of the plane of view. To fix this problem, 8 images were overlaid, to create a 
colored line to be more certain that measurements were taken from the same particle at different times instead of different particles moving in and out of the plane (Figure 15).

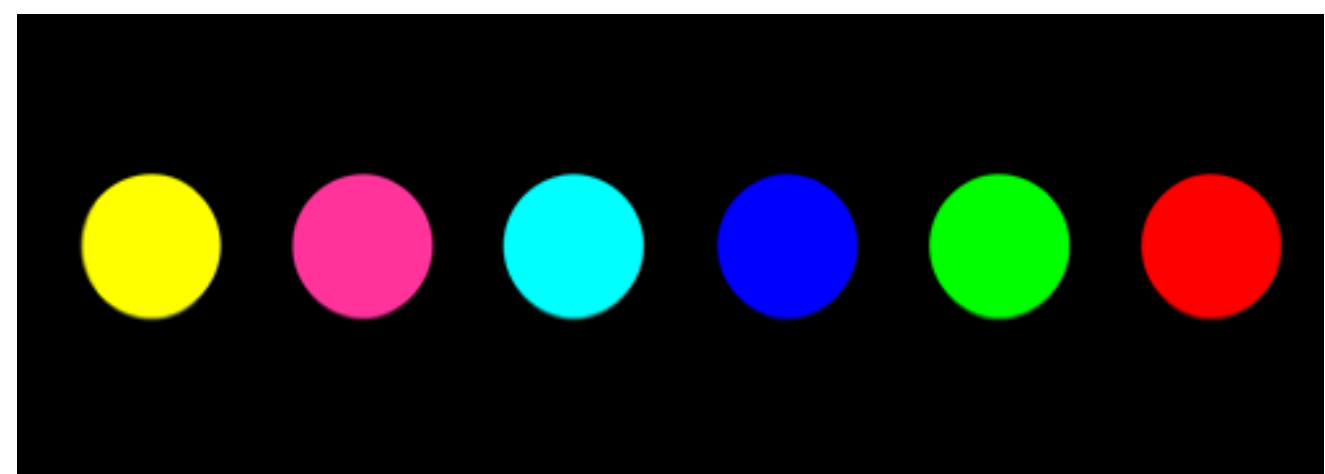

Figure 15. PIV image analysis. Example overlay of 8 images in different colors to allow for PIV measurements.

The $\mathrm{x}$ and $\mathrm{y}$ pixel location and the $\mathrm{x}, \mathrm{y}$, and $\mathrm{z}$ color values for these moving particles were recorded and analyzed in Excel. The color values were used to match the particle with one of the 8 images. This determined the time at which the particle was in that position (Figure 16). In addition, a line was fit to model the wall, so a velocity profile could be created to determine how far the particle was from the wall.

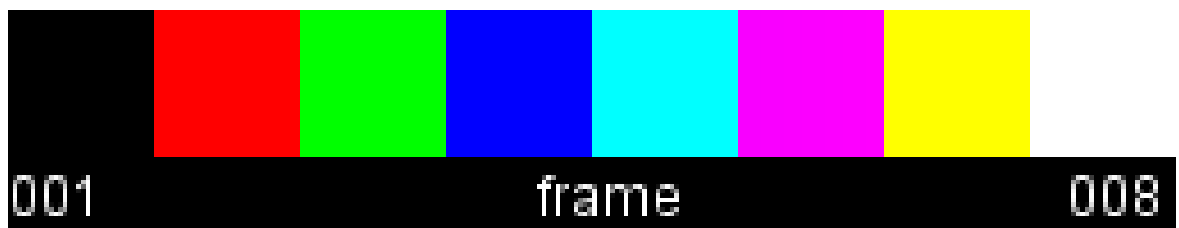

Figure 16. Color coder. Time-lapse color coder used to analyze stacks of 8 images that had been overlaid in different colors.

\subsection{RESULTS}

\subsubsection{Pressure}

Results were analyzed for 0.5 and $0.75 \mu \mathrm{L} / \mathrm{min}$ (Figure 17). Using JMP statistical software, data indicated no significant velocity difference between different 
regions along the channel, so the parabolic velocity profile is not present. The dimensions of the channel, being wide and thin, caused the parabolic profile to only be apparent very near the wall of the channel (Figure 18). However, this area had many sticking particles so this was not verifiable.

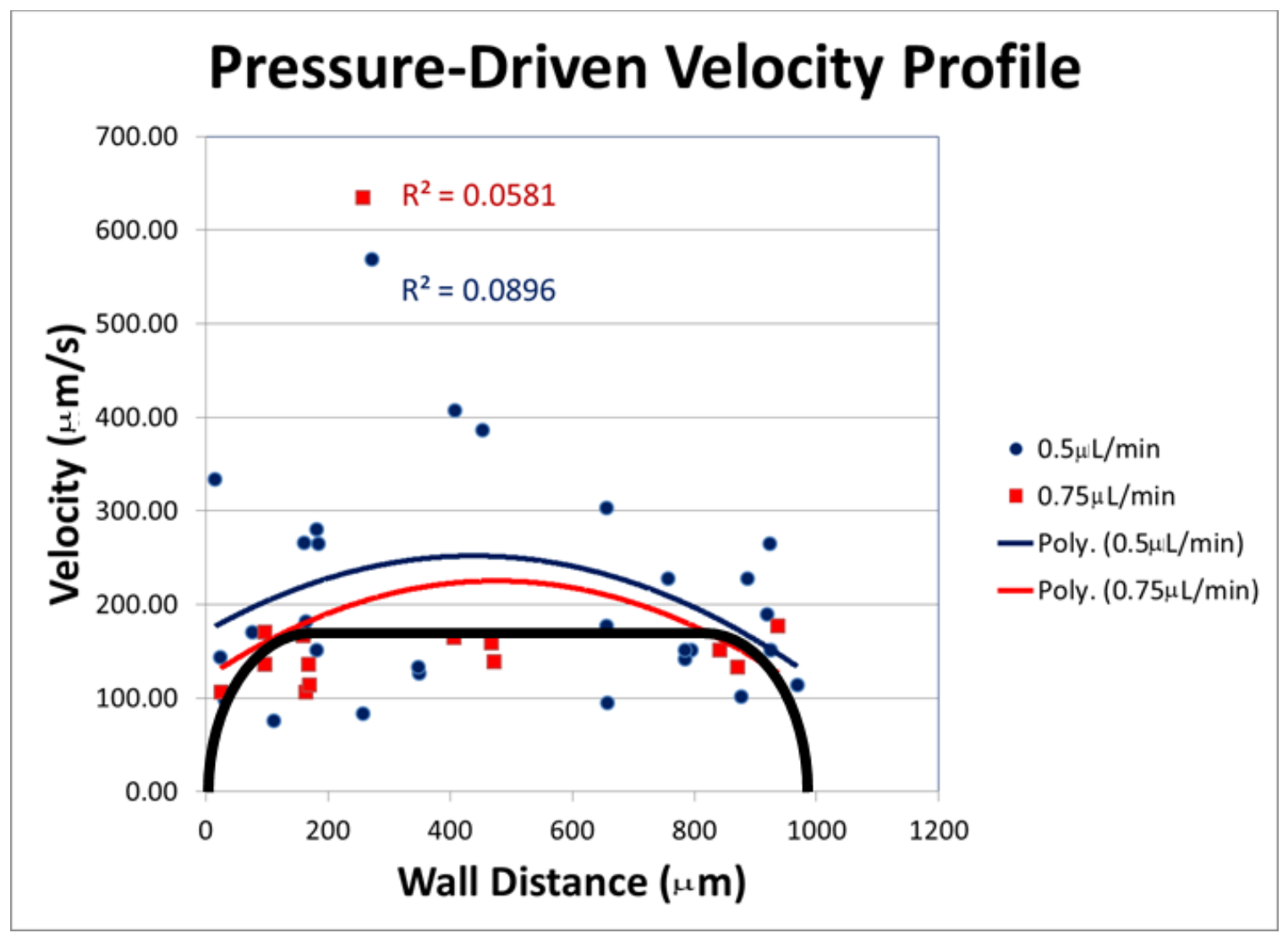

Figure 17. Graph of pressure-driven results. Pressure-driven results for $0.5 \mu \mathrm{L} / \mathrm{min}$ (blue) and $0.75 \mu \mathrm{L} / \mathrm{min}$ (red). For a wide and flat channel the parabolic profile would only be apparent near the wall of the channel (black). 


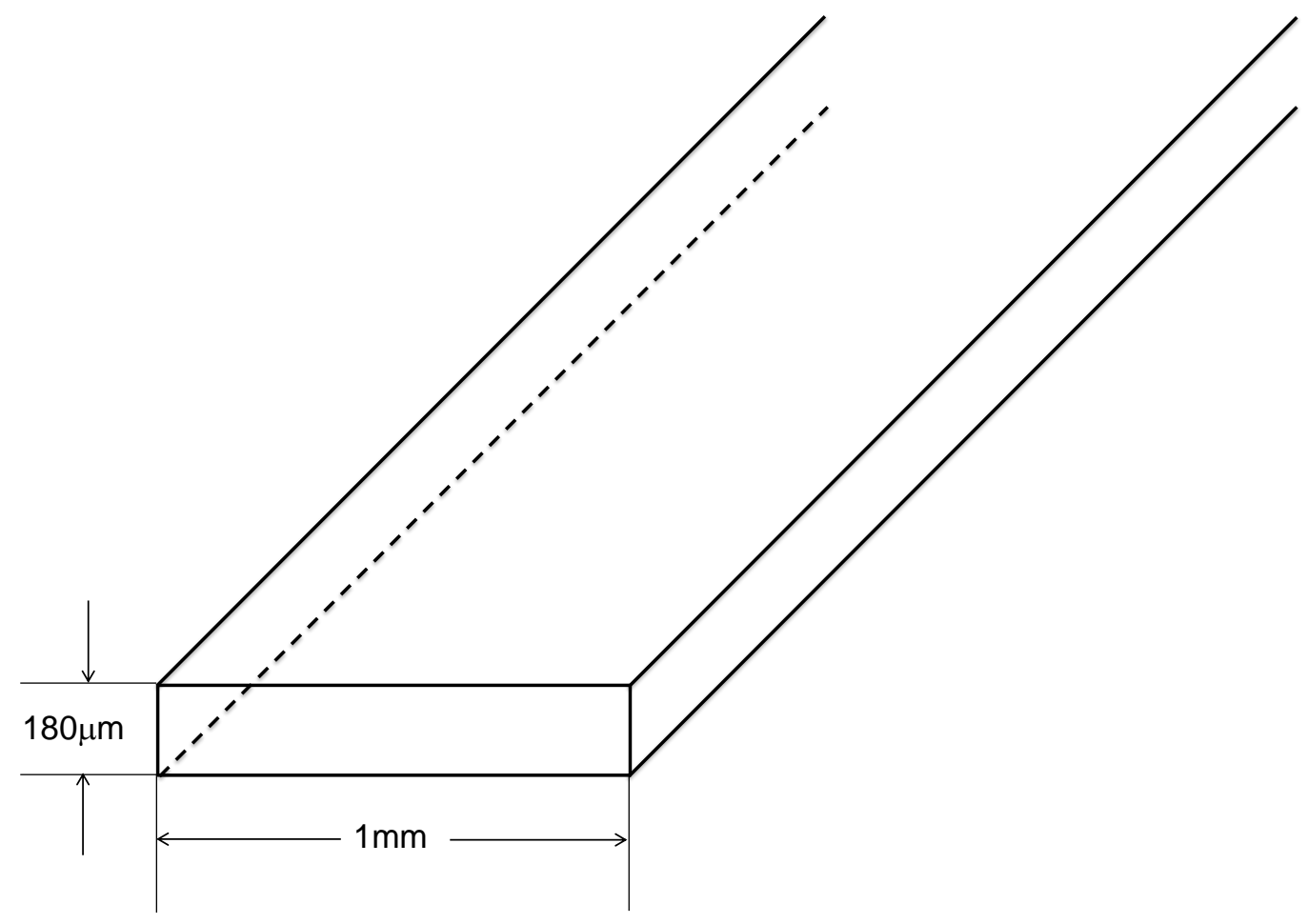

Figure 18. Drawing of pressure driven PDMS channel.

The overall average velocities conflict with logic (Table II). The $0.75 \mu \mathrm{L} / \mathrm{min}$ average is less than the $0.5 \mu \mathrm{L} / \mathrm{min}$ average. Inaccuracies in the syringe pump were hypothesized to be the cause. In addition, there is significant variation in the data. This is probably from wall interactions since the depth of the particle cannot be determined when imaging. Finally, the calculated velocities are different from the expected velocities. This was also possibly from inaccuracies in the syringe pump.

Table II. Pressure-driven results summary by laser confocal microscope. The average velocity and its standard deviation was calculated for two measured flow rates along with their expected velocities.

\begin{tabular}{cccc}
\hline $\begin{array}{c}\text { Pump } \\
\text { Rate } \\
(\mu \mathrm{L} / \mathrm{min})\end{array}$ & $\begin{array}{c}\text { Average } \\
\text { Velocity } \\
(\mu \mathrm{m} / \mathrm{s})\end{array}$ & $\begin{array}{c}\text { Standard } \\
\text { Deviation }\end{array}$ & $\begin{array}{c}\text { Expected } \\
\text { Velocity } \\
(\mu \mathrm{m} / \mathrm{s})\end{array}$ \\
\hline $\mathbf{0 . 5}$ & 205.83 & 112.92 & 46.3 \\
$\mathbf{0 . 7 5}$ & 178.16 & 133.30 & 69.44 \\
\hline
\end{tabular}




\subsubsection{Syringe Pump}

Since the pressure driven results were consistently higher than expected the syringe pump was suspected to not be accurate for low flow rates. Using a Denver Instruments analytical scale, measurements were taken every 5 minutes for 90 minutes (Figure 19).

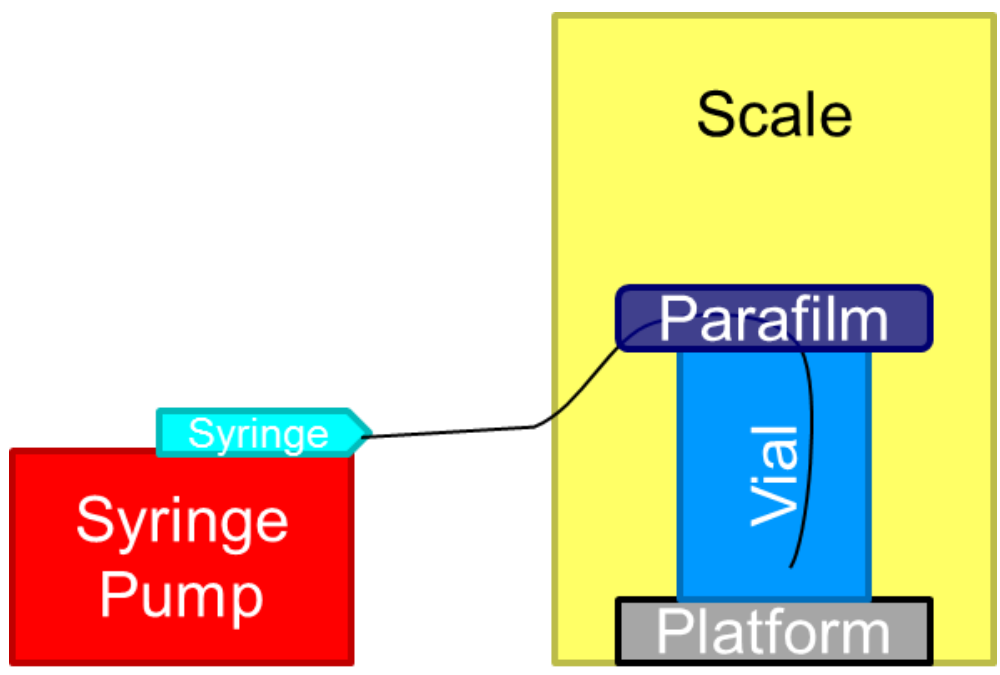

Figure 19. Setup for syringe pump verification.

This data was graphed in Excel and fit with a linear regression line (Figure 20).

The slopes showed the true flow rate for the syringe pump setting. The flow rates were actually a little lower than the setting which makes the data make even less sense. There could possibly still be some pulsing in the syringe pump which might explain some of the inconsistencies seen but not show in the overall flow rate. 


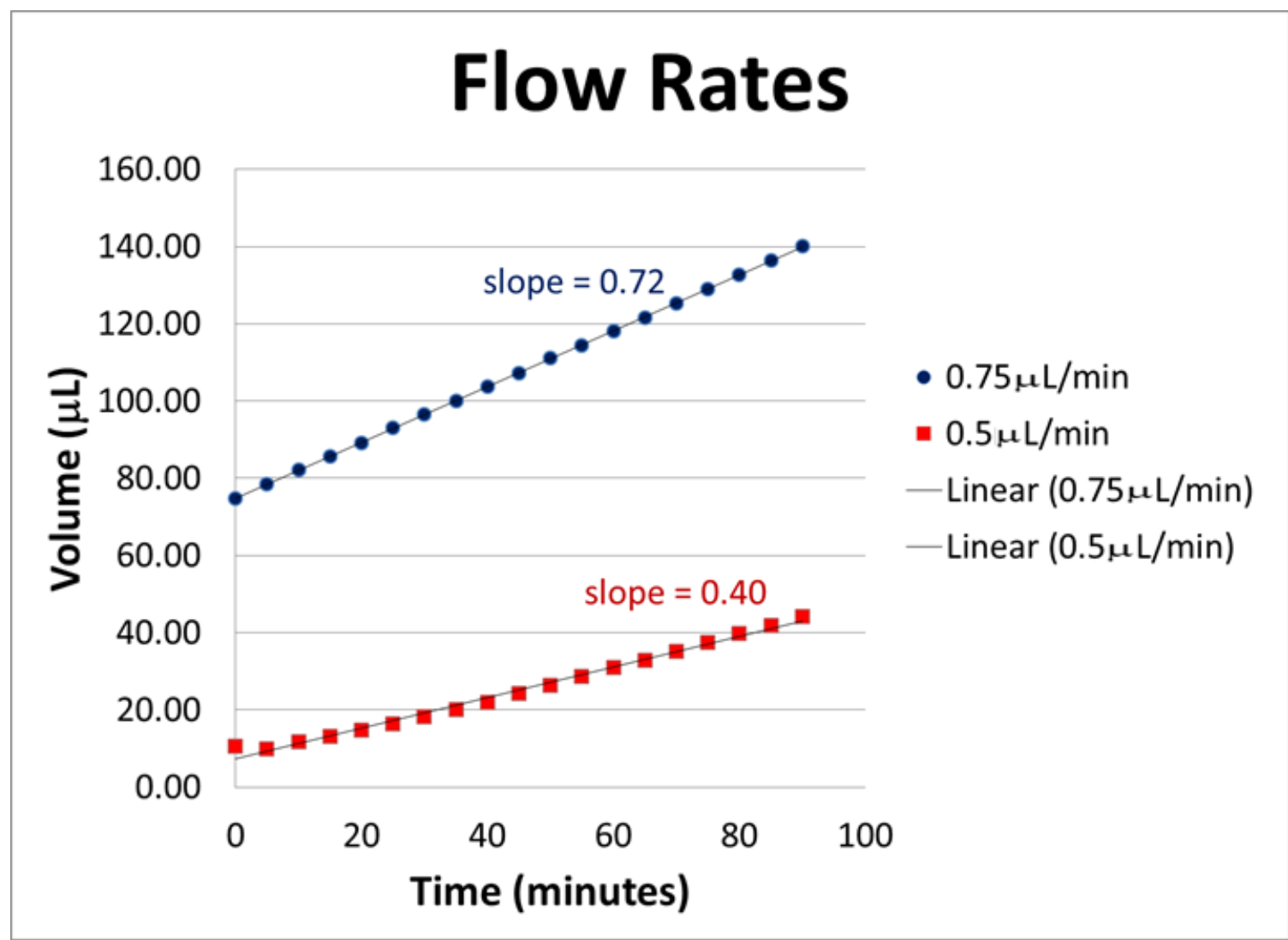

Figure 20. Results of syringe pump verification.

\subsubsection{Electro-Kinetic}

There were many issues with the electro-kinetic flow, and none of the obtained measurements were reliable. Often flow would switch directions at random intervals. Charge build up was thought to be causing directions to change. Pressure was added as a base to try to remove this buildup within the channel and get a more consistent flow. Bubbles also appeared easily when testing electro-kinetic flow, especially when adding pressure to remove the reverse flow direction (Figure 21). This also happened with pressure-driven flow but not as often. The parabolic velocity profile associated with pressure-driven flow is very apparent with the bubble. The bubble was also very good at clearing out the florescent particles from the channel, even many of the ones that were stuck. 
A

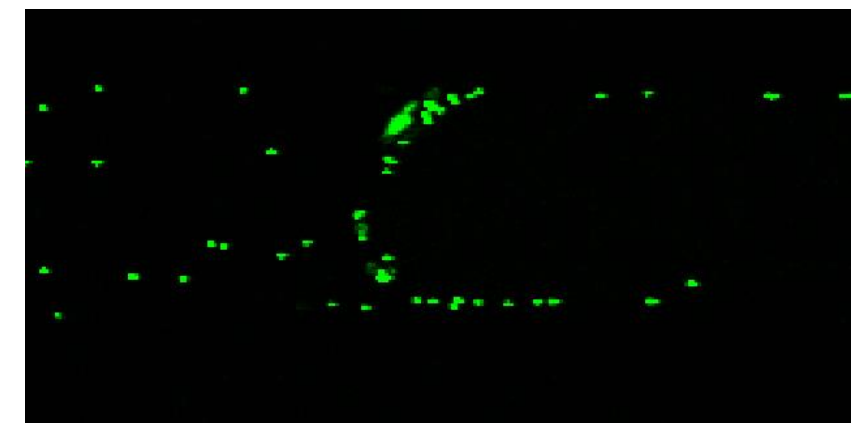

B

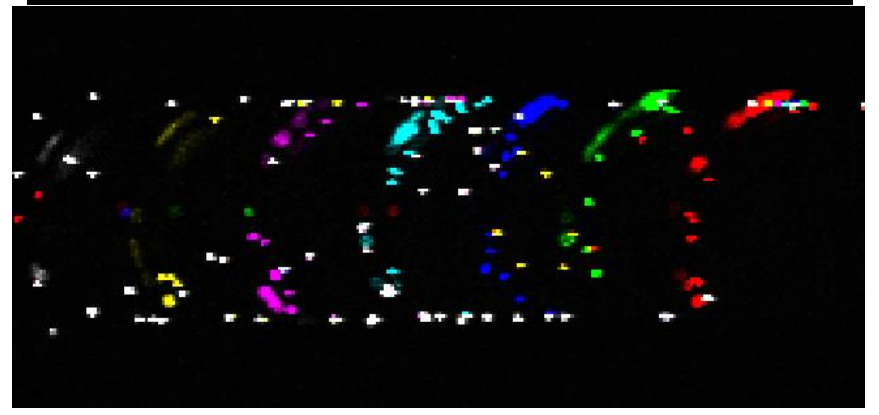

Figure 21. Bubble in channel. Bubble traveling through the channel with $0.25 \mu \mathrm{L} / \mathrm{min}$ of pressure and $50 \mathrm{~V}$. The voltage appears to have no impact on the flow.

Research showed that bubbles are often introduced in microfluidics from connecting tubing or moving the channel, causing the release of absorbed gas. PDMS has the ability to absorb gas from its surrounding atmosphere, which it can then release during use. In addition changes in temperature, pressure, surface properties of the channel, and properties of the fluid within the channel can cause bubbles to grow. A simple bubble trap can be created at the inlet where bubbles can flow out of the way of the channel. This provides temporary help since the bubble is not completely removed from the system. However, the length of time depends on the flow rates and the size of the bubble trap [27].

\subsection{LESSONS LEARNED FROM FOUNDATIONAL EXPERIMENT}

The fabrication process worked well and can be used for a variety of channels.

Pressure-driven flow in the PDMS channel did not show a parabolic profile since the width to height ratio is large. In addition, there was variation between the 
particles and the data was different from expected. Electro-kinetic driven flow was greatly influenced by air bubbles in the system and surface interactions. Finally, the laser confocal microscope did work as a way to perform PIV on microfluidic chips. 


\section{Chapter 4 Materials, Methods, and Procedures}

Since particles appeared to be sticking to the PDMS, channels were bought of varying materials and dimensions. Various particle solutions and concentrations were used to help differentiate particles on different microscopes. Pressure measurements were taken to prevent breaking channels. A microscope from LabSmith (S/N\#: S340R3A150 0109) with UScope software to read the velocity of particles moving in different parts of the channel was used to get pressure driven measurements.

\subsection{MICRO-FLUIDIC CHIP SELECTION}

New channels were purchased from Translume, Cidra, and LabSmith of varying dimensions and materials:

\subsubsection{Translume}

Translume channels are made of fused silica for optical clarity and low autofluorescence. This material reacts with hydrofluoric acid and potassium hydroxide at high temperatures. The bottom up version of these channels was used to be compatible with an inverted microscope. These channels are made with almost perfect $90^{\circ}$ corners. One millimeter graduated lines run along the channel which help locate the channel using higher magnifications. Luer connectors are used to connect tubing to the channel.

A cross-channel with cross-sectional area dimensions of $100 \mu \mathrm{m}$ by $100 \mu \mathrm{m}$ was received from Dr. Laiho (Figure 22) [28]. High pressure was applied at the inlet to 
remove an apparent clog, which caused the back glass to break. A pressure sensor was purchased to avoid this happening again.
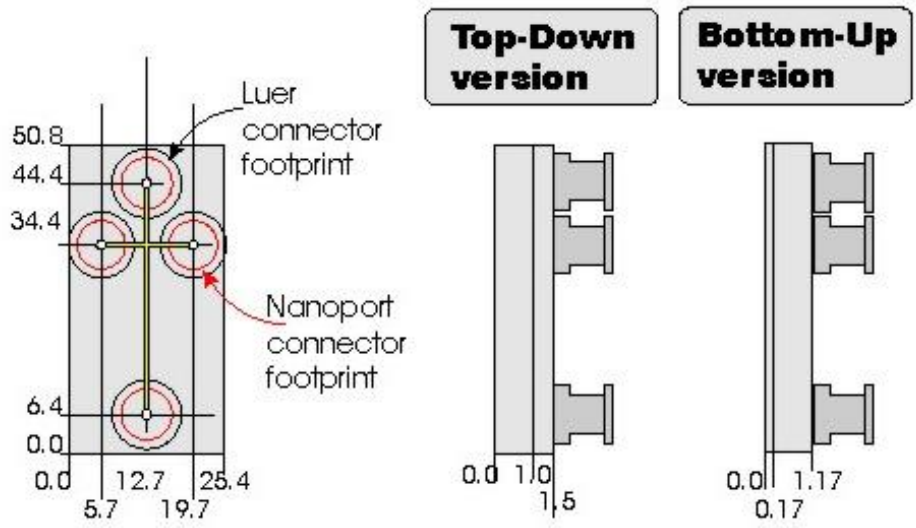

Not to scale All dimensions in $\mathrm{mm}$.

Figure 22. Layout of Translume cross-channel.

A straight-channel with cross-sectional area dimensions of $300 \mu \mathrm{m}$ by $300 \mu \mathrm{m}$ was purchased (Figure 23) [29].

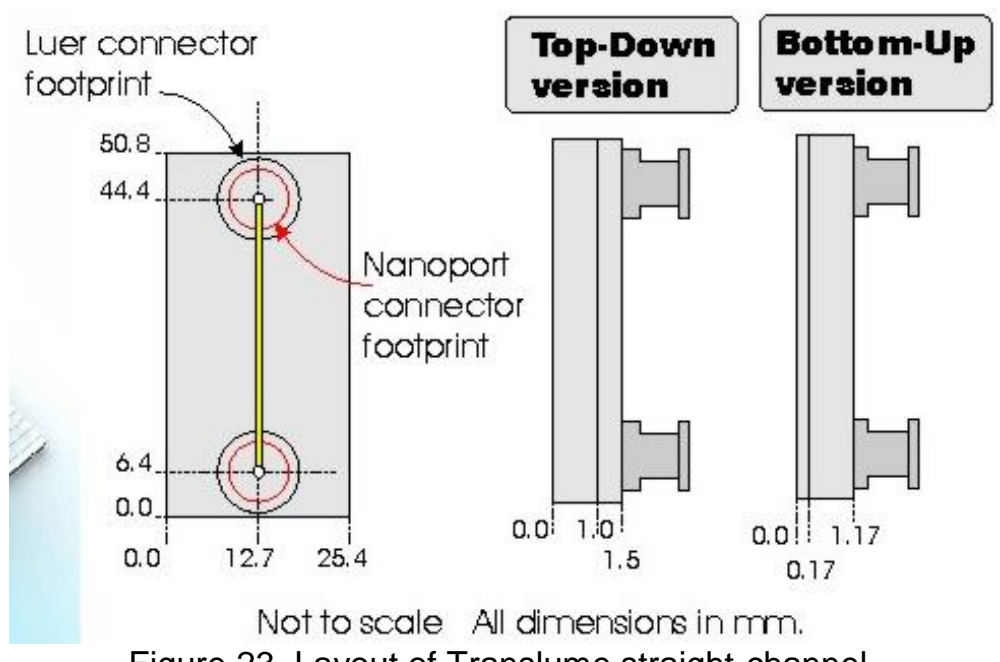

Figure 23. Layout of Translume straight-channel.

\subsubsection{Cidra}

Two Cidra single-channel flow cells with cross-sectional area dimensions of $2.5 \mathrm{~mm}$ by $100 \mu \mathrm{m}$ were purchased (Figure 24) [30]. This channel is made of 
borosilicate glass which is a fairly inexpensive, low auto-fluorescence material with good machinability [15]. This channel does not come with pre-connected connectors, so LabSmith bonded-port connectors were epoxied to the inlet and outlet holes. More detail on bonding connectors is discussed below.

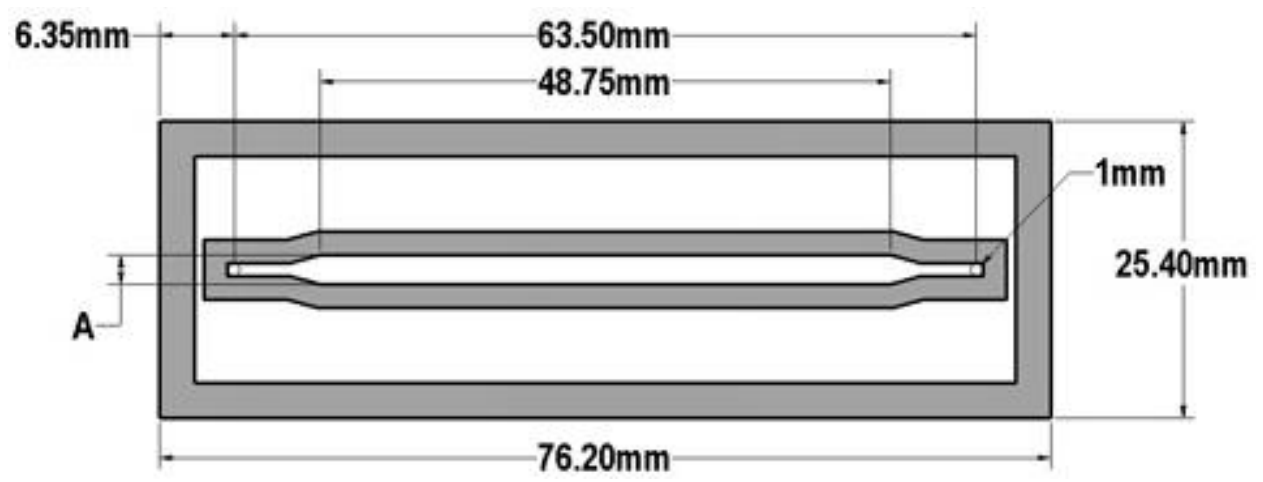

Figure 24. Layout of Cidra single-channel flow cell.

\subsubsection{LabSmith}

Three LabSmith cross-channel through-holes chips with cross-sectional area dimensions of $100 \mu \mathrm{m}$ by $100 \mu \mathrm{m}$ were purchased, two made out of PMMA and one made from TOPAS. Under an optical microscope, it appeared that this channel may not be a perfect square, but have a trapezoidal shape (Figure 25). These chips did not come with pre-connected connectors, so LabSmith bondedport connectors were epoxied to the inlet and outlet holes. Again, more detail on bonding connectors is discussed below. 


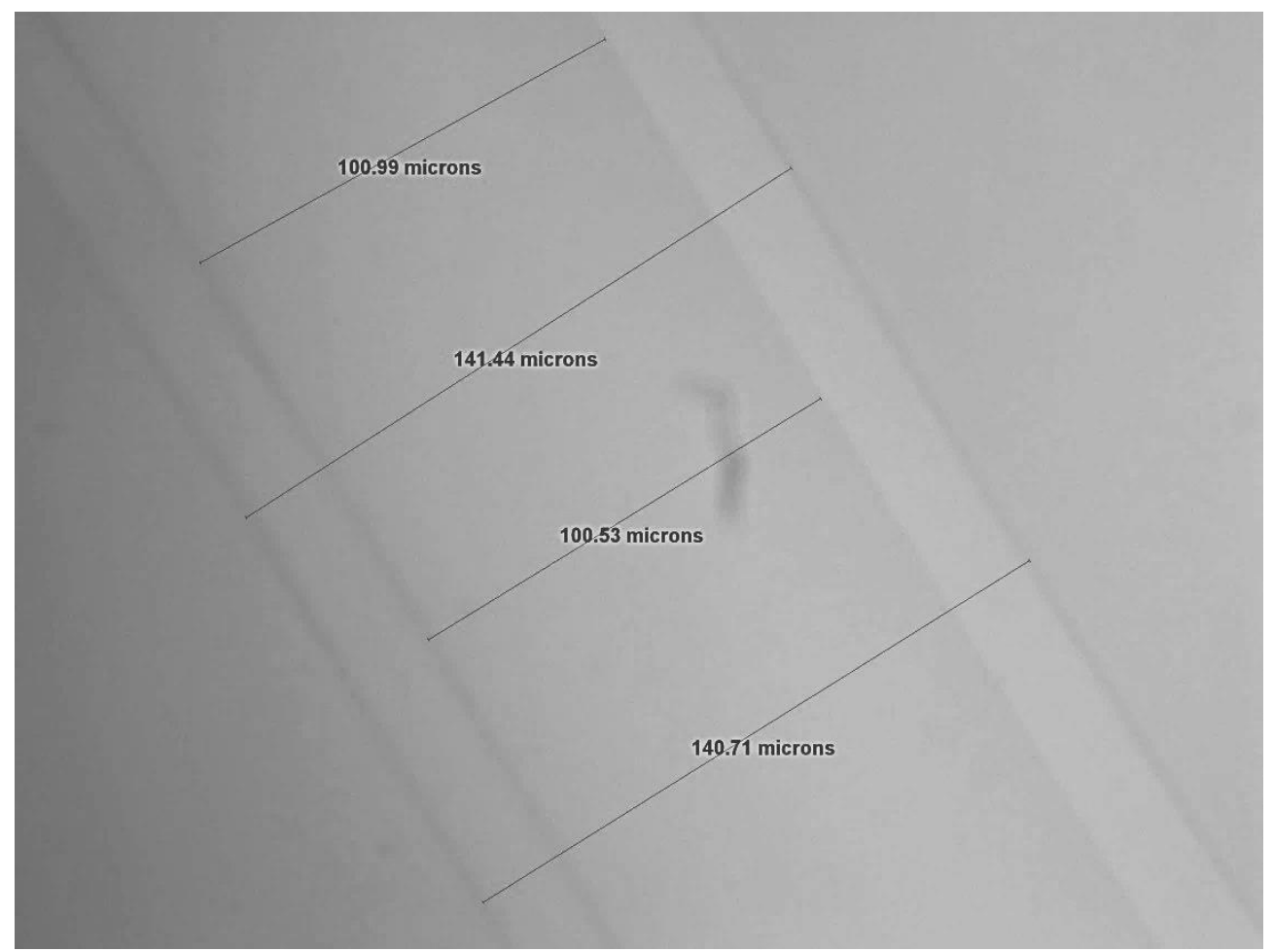

Figure 25. LabSmith PMMA optical microscope image. Optical microscope image of PMMA LabSmith cross-channel through-hole chip taken at 50x magnification. The image shows the base dimensions of the trapezoidal channel. One base has an average dimension of $100.76 \mu \mathrm{m}$ and the other base has an average dimension of $141.08 \mu \mathrm{m}$.

\subsubsection{Cleaning}

Cleaning procedures were established to avoid previous issues with particles sticking to the surface of the channel over time, making it more difficult to see moving particles. Although the channels were rinsed with DI water after use, the particles still stuck to the PDMS. It was desired to determine another cleaning method that would minimize particles sticking to the purchased channels. The Cidra channels came with cleaning instructions that were adapted to use on other channels [31]. The multiple steps are as follows:

1. Obtain proper safety wear.

2. Obtain a less than $5 \%$ by volume $\mathrm{KOH}$ solution. Inject solution into channel and let stand for 10 minutes while agitating every 3 minutes.

3. Flush $\mathrm{KOH}$ from channel, neutralize, and dispose. 
4. Flush channel with DI water and blow with nitrogen gas. Repeat three times.

5. Flush channel with IPA water and blow with nitrogen gas. Repeat three times.

6. Flush channel with filtered DI water and store.

For the Translume, Cidra and TOPAS LabSmith channels, this cleaning process worked without any issues as long as the $\mathrm{KOH}$ solution was not heated.

However, the PMMA LabSmith channel stress cracked and leaked when using IPA. Skipping this step did not cause significant issues with the channel and still cleaned the channel adequately.

This cleaning method worked well for pressure-driven flow; however, while testing electro-kinetic driven flow, the polystyrene particles clumped together and stuck to the wall, interfering with visually seeing particle movement. Various solvents were used to try to remove the particles from the channel. First, acetone was pumped through the channel carefully since it can react with the connectors, but this also did not work. Second, TritonX, a surfactant, was pumped through the channel, and this still did not work. Finally, a Cole Parmer $\mathrm{pH} 4.01$ buffer solution was used along with suspending the channel in a sonic bath (Figure 26). This removed most of the particles form the channel. Most likely, the PDMS wetting solution caused some branching between the charge of the channel wall and the charge of the polystyrene particles, causing them to stick. 


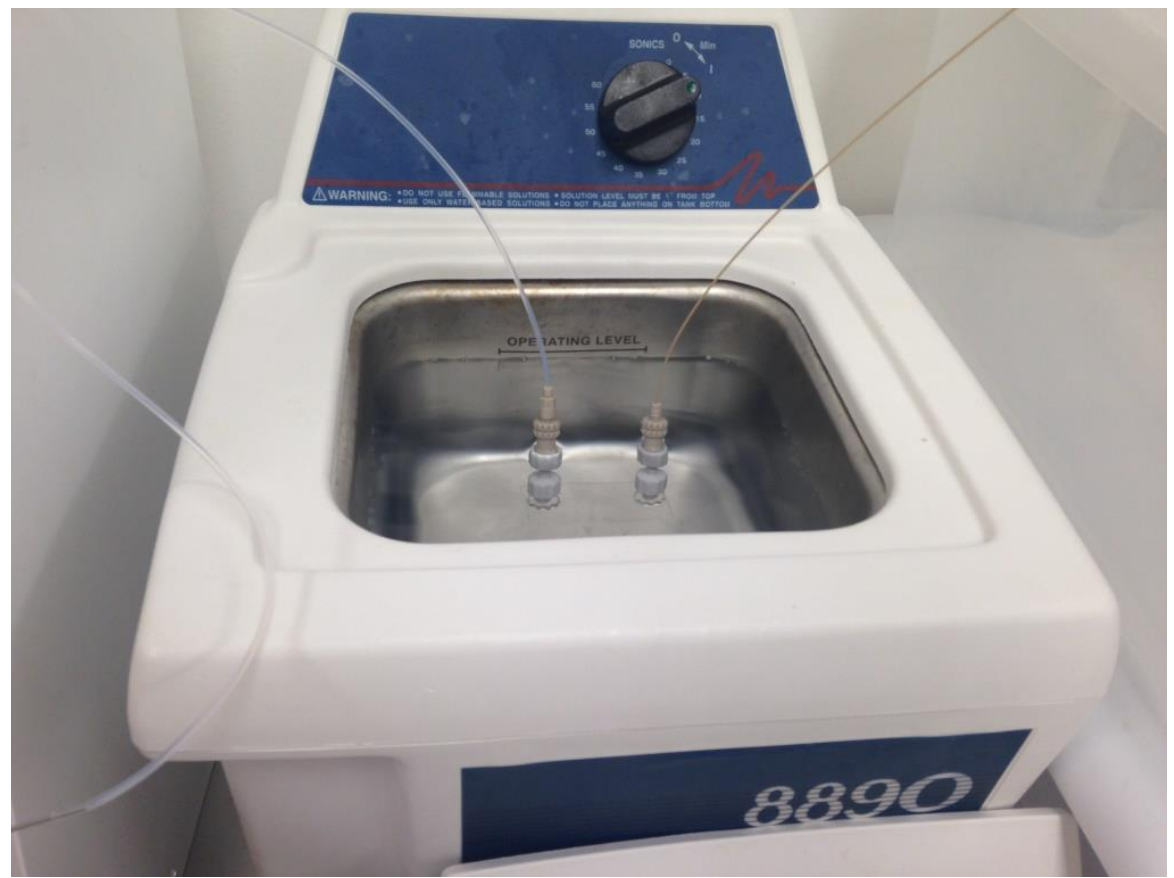

Figure 26. Translume channel cleaning. Translume channel suspended in sonic bath to remove particles stuck to channel walls.

\subsubsection{Connectors}

LabSmith bonded-port connectors include suggested instructions for installation [32]. An epoxy, provided by LabSmith, was mixed in a 2:1 ratio. LabSmith suggests applying a small amount to the bottom of the connector and then placing on the channel hole. A pin tip was used to apply the epoxy. It was found that it was very hard to get the connector and hole to align properly with one chance and many of the connectors were clogged. Instead, the connectors were placed first and affixed with Scotch tape (Figure 27). The epoxy was then placed around the outer edge of the connector. It does not look pretty on top but the channel is viewed from the bottom, so the top does not really matter and the connectors were not clogged. 

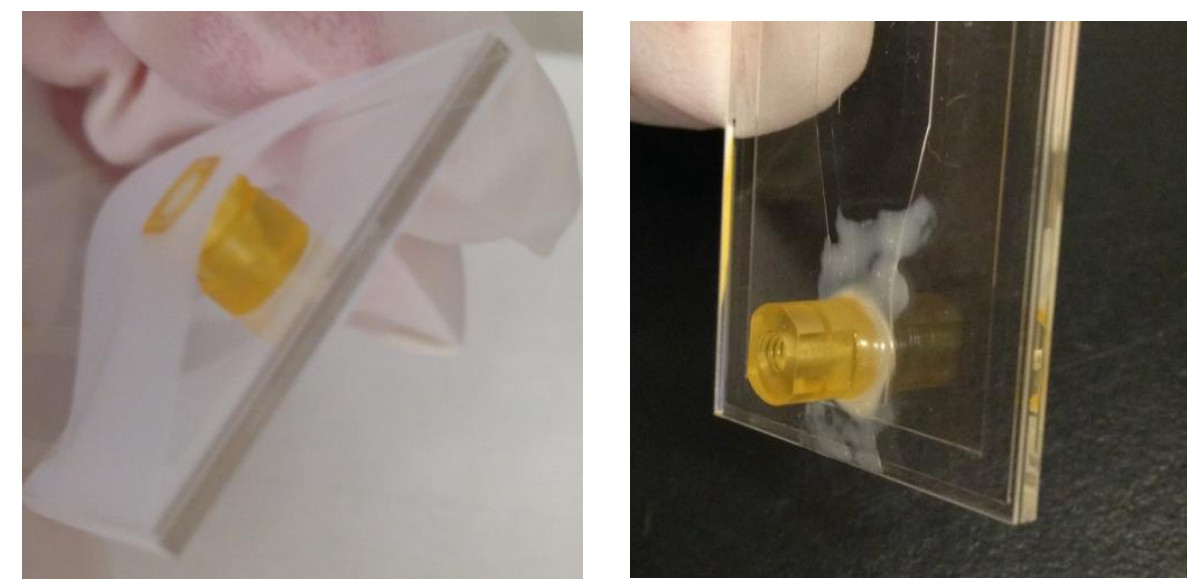

Figure 27. Attaching connectors. Left: Aligned connector on channel hole. Right: Epoxied connector on channel hole.

\subsection{PARTICLE SOLUTION}

\subsubsection{Bubble Avoidance}

A PDMS wetting solution was purchased from Cidra because of its hydrophobicity [33]. This solution also helps wet glass. A wetting solution was required to avoid bubbles forming in the channel. A bubble was purposefully introduced into a channel with DI water with and without the wetting solution (Figure 28). The channel with wetting solution appears to have a layer covering the channel. In addition, as the bubble moved through the channel the walls of the bubble would move in and out. The channel without wetting solution just appears to stay still with no sign of movement as the bubble moved through. In addition, large droplets of water were left within the middle of the channel. This wetting solution was added to particle solutions in the recommended low concentrations, $0.001 \%$ to $0.1 \%$, to improve movement through the channel. Some bubbles were still seen within the channel, but they moved through, although more slowly than the particles. 

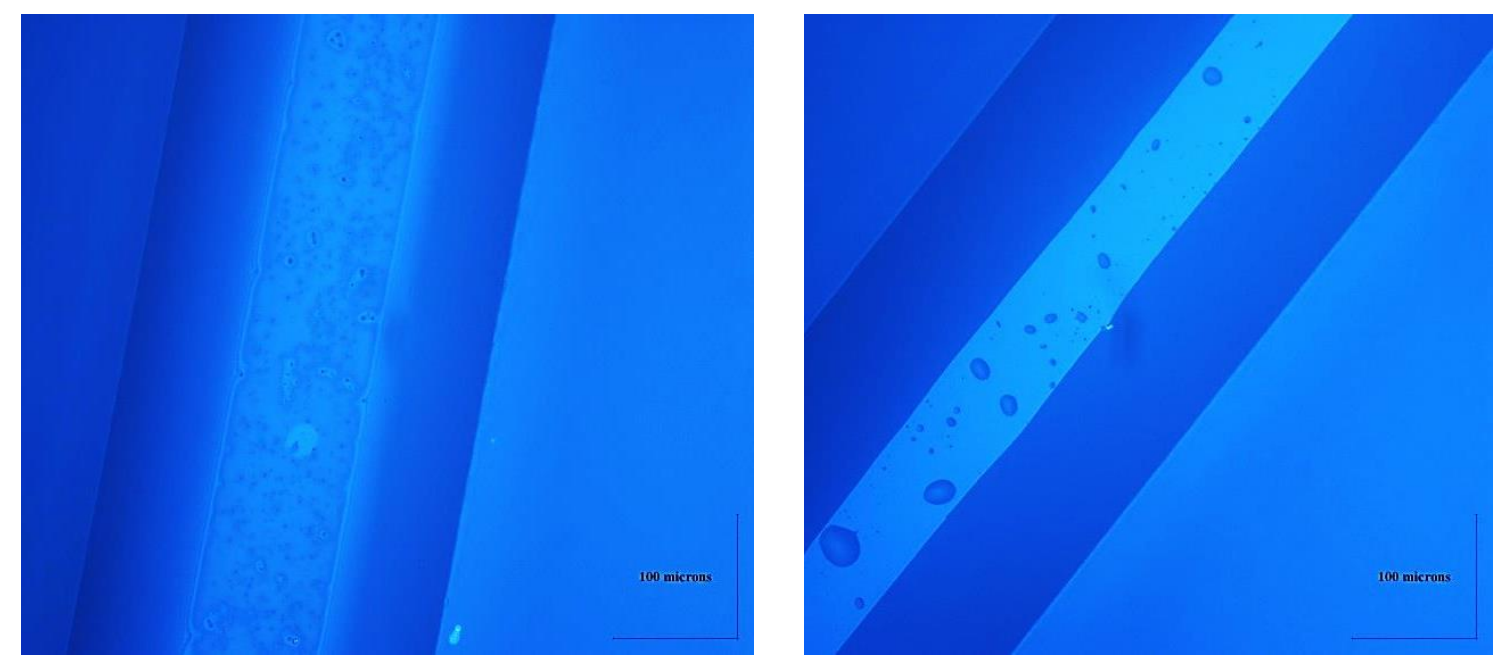

Figure 28. Effect of wetting agent on bubble in channel. Left: Channel bubble with wetting solution. Right: Channel bubble without wetting solution.

Appendix A details the process for and results of analyzing the effect of this wetting agent.

\subsubsection{Particle Selection}

Multiple beads were purchased of various sizes and fluorescence to determine which was best to work with on the microscope.

- $10 \mu \mathrm{m}$ Invitrogen fluoSpheres polystyrene microspheres with an excitation wavelength of $430 \mathrm{~nm}$ and an emission wavelength of $465 \mathrm{~nm}$ with a concentration of $3.6 \times 10^{6}$ beads $/ \mathrm{mL}$ [34]. The concentration of these beads was significantly lower than the other beads, but when they did show up they appeared to be moving slower than the smaller spheres. It was also harder to take measurements close to the wall with larger beads. This selection was rejected because the larger size allowed for more wall interactions that influenced the particles velocity.

- $1 \mu \mathrm{m}$ Invitrogen fluoSpheres polystyrene biotin-labeled microspheres with an excitation wavelength of $505 \mathrm{~nm}$ and an emission wavelength of $515 \mathrm{~nm}$ with a concentration of $1 \%$ solids [35]. The biotin-label can have hydrophobic regions that might stick to surfaces but this did not appear to be the case within the glass channels. These were rejected because the small size made it difficult to observe the beads on the microscope.

- $1 \mu \mathrm{m}$ Invitrogen fluoSpheres polystyrene carboxylate-labeled microspheres with an excitation wavelength of $580 \mathrm{~nm}$ and an emission wavelength of 
$605 \mathrm{~nm}$ with a concentration of $2 \%$ solids [36]. The carboxylate-label creates charges on the surface of the spheres that might affect the way these spheres interact with surfaces which may not be desirable for pressure-driven flow but is necessary for electro-kinetic flow. For the glass channels, there did not appear to be any excessive sticking to the walls that would interfere with measurements. However, these beads were also rejected due to their small size and difficulty focusing.

- $2 \mu \mathrm{m}$ Intivrogen fluoSpheres polystyrene carboxylate-labeled microspheres with an excitation wavelength of $505 \mathrm{~nm}$ and an emission wavelength of $615 \mathrm{~nm}$ with a concentration of $2 \%$ solids [37]. These beads were easier to focus on and appeared to move about the same as the $1 \mu \mathrm{m}$ beads, but the dilution that was made was too dilute to use with the LabSmith microscope, but a good concentration to view by eye on the laser confocal microscope. These particles were used for most of the pressure-driven flow measurements, but stuck to the walls during electro-kinetic flow.

- $1 \mu \mathrm{m}$ Invitrogen fluoSpheres polystyrene microspheres with excitation wavelength of $580 \mathrm{~nm}$ and emission wavelength of $605 \mathrm{~nm}$ with a concentration of $10^{10}$ beads $/ \mathrm{mL}$ [38]. These beads were diluted to a concentration of about 99 million beads $/ \mathrm{mL}$ in a $\mathrm{pH} 7.00$ buffer solution. These were slightly hard to see with the LabSmith microscope, but would have minimal interactions with the wall. Although, these particles were small, they did not have any surface groups that might have been responsible for the previous particles sticking to the wall and were used for all electro-kinetic measurements.

Equation 14 was used to convert the concentrations from those given to something comparable between the solutions, where $C$ is the given concentration of suspended beads in $\mathrm{g} / \mathrm{mL}(\mathrm{ex} .2 \% \rightarrow 0.02 \mathrm{~g} / \mathrm{mL}), \phi$ is the diameter of the microspheres in microns, and $\rho$ is the density of the bead polymer in $\mathrm{g} / \mathrm{mL}$ $(1.05 \mathrm{~g} / \mathrm{mL}$ for PS). Differing concentrations were made to determine which concentration was the best to be used with the microscopes. Visually, for use on the laser confocal microscope, the best solution has about $3.4 \times 10^{6}$ beads $/ \mathrm{mL}$, with the $2 \mu \mathrm{m}$ beads. The LabSmith microscope requires a much higher concentration for the probes to obtain many measurements within a reasonable 
amount of time; for example, $9.9 \times 10^{7}$ beads $/ \mathrm{mL}$, with the $1 \mu \mathrm{m}$ beads or $9.7 \times 10^{7}$ beads $/ \mathrm{mL}$, with the $2 \mu \mathrm{m}$ beads.

$$
\begin{aligned}
& \text { Equation 14. Concentration conversion between solutions. } \\
& \qquad \frac{\text { \#of microspheres }}{m L}=\frac{6 C \times 10^{12}}{\rho \times \pi \times \phi^{3}}
\end{aligned}
$$

\subsection{Pressure-Driven Flow Experimental Procedures}

\subsubsection{Syringe Pumps}

Two types of syringe pumps were used to obtain pressure driven flow. Both syringe pumps had motor pulsing issues at low flow rates. The motor when running is audible, so to some extent the pulsing of the motor can be heard. Originally a New Era Syringe Pump was used, but it was found that at the low flow rates the noise from the pulsing motor overpowered the trends seen in the data, so a Harvard Apparatus Syringe Pump was used to try to minimize this issue. The Harvard Apparatus Syringe Pump still had the issue, but at lower flow rates, so some reasonable data was able to be collected.

\section{New Era Syringe Pump}

New Era Syringe Pumps (Model\#: NE-300) are easily accessed in the micro-fab lab. These pumps are for infusion only and can be used with a variety of different syringe sizes. With the $1 \mathrm{~mL}$ Norm-Ject syringe (inner diameter of $4.69 \mathrm{~mm}$ ), this pump has a rate limit of $0.01 \mu \mathrm{L} / \mathrm{min}$ to $651.00 \mu \mathrm{L} / \mathrm{min}$ [39]. 


\section{Harvard Apparatus Syringe Pump}

Harvard Apparatus Syringe Pumps (Catalog\#: 70-2211) are available in the microfluidics lab. These pumps can be used for either infusion or withdrawal and with many different syringe sizes. With a similar $1 \mathrm{~mL}$ syringe, this pump has a rate limit of $0.05 \mu \mathrm{L} / \mathrm{min}$ to $792 \mu \mathrm{L} / \mathrm{min}[40]$.

\subsubsection{Measuring Pressure}

Early on, a Translume cross-channel broke due to pressure while trying to remove a clog. A LabSmith pressure sensor starter kit was purchased to use with 1/16" tubing with a $250 \mathrm{kPa}$ and $800 \mathrm{kPa}$ sensor to avoid future breakage [41]. The Cidra channels had a maximum pressure of $45 \mathrm{psi}$, or $310 \mathrm{kPa}$. The $800 \mathrm{kPa}$ sensor was used until it was determined that the max flow rate on the syringe pump could not exceed $250 \mathrm{kPa}$, and the $250 \mathrm{kPa}$ sensor was used instead to obtain more accurate measurements.

In addition, due to inaccuracies of the syringe pumps and inconsistencies between syringe pumps, the pressure within a channel was measured at multiple different flow rates from $5 \mu \mathrm{L} / \mathrm{min}$ to $500 \mu \mathrm{L} / \mathrm{min}$. A $1 \mathrm{~mL}$ Norm-Ject syringe from Henke Sass Wolf with an inner diameter of $4.69 \mathrm{~mm}$ was used for all the pressure measurements with filtered DI water.

A $250 \mathrm{kPa}$ pressure sensor was used for measurements and connected to the channel and syringe through tubing and a Tee Interconnect on a Component Breadboard. The sensor was connected to the Valve Manifold and then the Electronic Interface Board which connected to the computer with the uProcess 
software. The software was set to measure with a $20 x$ gain and a sampling rate of $1.8 \mathrm{kS} / \mathrm{s}$ (Figure 29) [42].

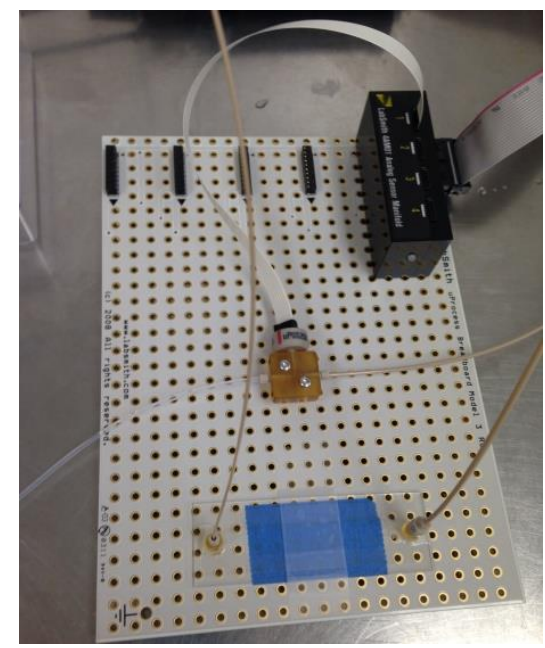

Figure 29. Pressure measurement setup. Set-up of Cidra channel with pressure-sensor system. Tubing is run from the syringe pump to a T-connector and from the T-connector to the channel. A pressure sensor is also attached to the T-connector and to the computer to be logged. Set up is similar for other channels measured.

The pressure reached a fairly steady state quickly (Figure 30, Figure 31, Figure 32, Figure 33).To ensure this was a truly steady state for a given flow rate, the flow rate was applied for a few minutes to allow the pressure to stabilize. Measurements were taken at $5 \mu \mathrm{L} / \mathrm{min}, 50 \mu \mathrm{L} / \mathrm{min}, 100 \mu \mathrm{L} / \mathrm{min}, 250 \mu \mathrm{L} / \mathrm{min}$, and $500 \mu \mathrm{L} / \mathrm{min}$. The LabSmith Topas and PMMA channels were not measured at $500 \mu \mathrm{L} / \mathrm{min}$ due to high pressures. The syringe was allowed to pump for 15 minutes for the $5 \mu \mathrm{L} / \mathrm{min}$ and $50 \mu \mathrm{L} / \mathrm{min}, 10$ minutes for the $100 \mu \mathrm{L} / \mathrm{min}, 5$ minutes for the $250 \mu \mathrm{L} / \mathrm{min}$, and 2 minutes for the $500 \mu \mathrm{L} / \mathrm{min}$. Shorter times were required for the larger flow rates since the syringe emptied and needed to be refilled. 


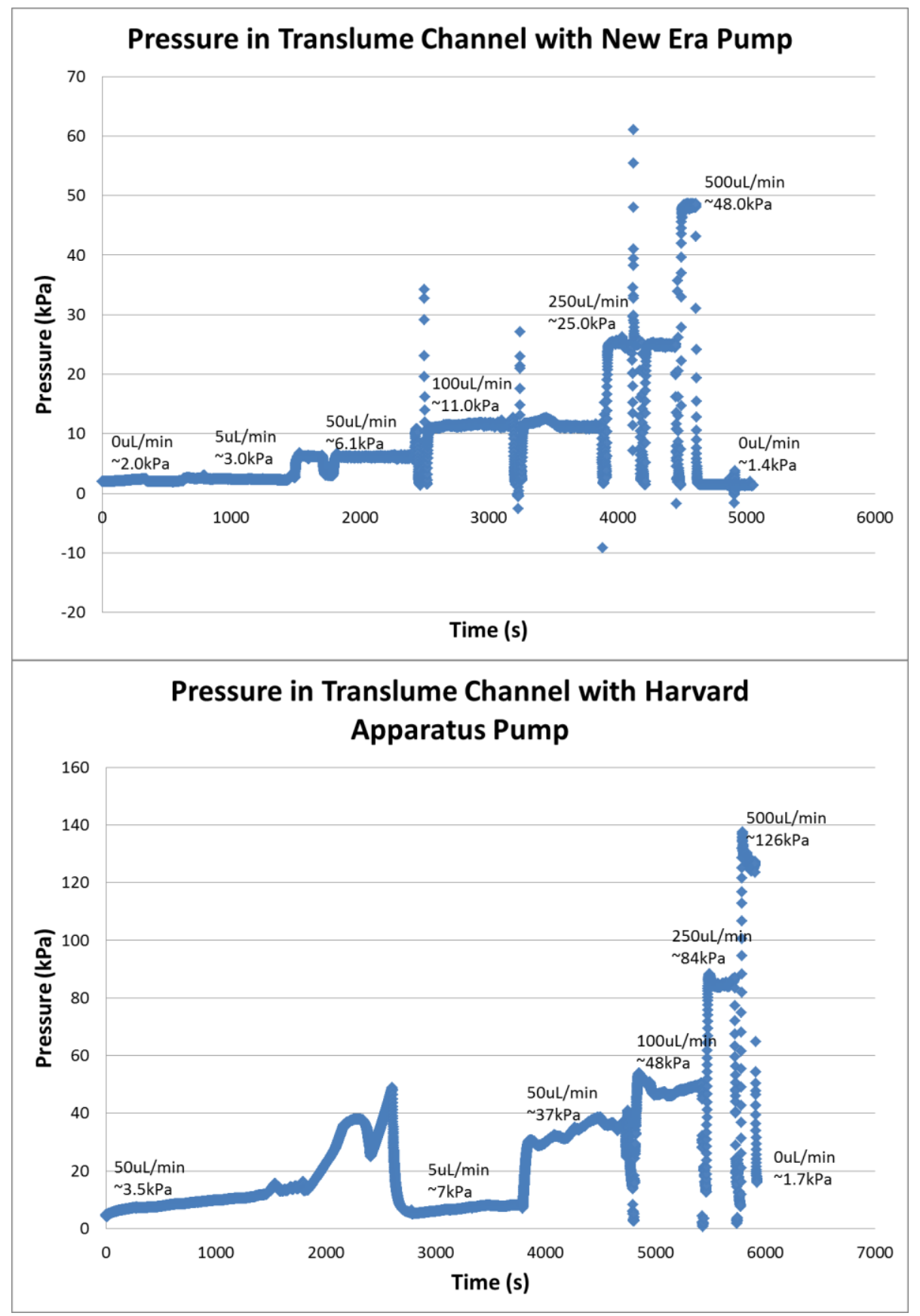

Figure 30. Pressure measurement for Translume channel. Output from LabSmith pressure sensor with New Era and Harvard Apparatus syringe pump. Drops during testing are from refilling the syringe. 


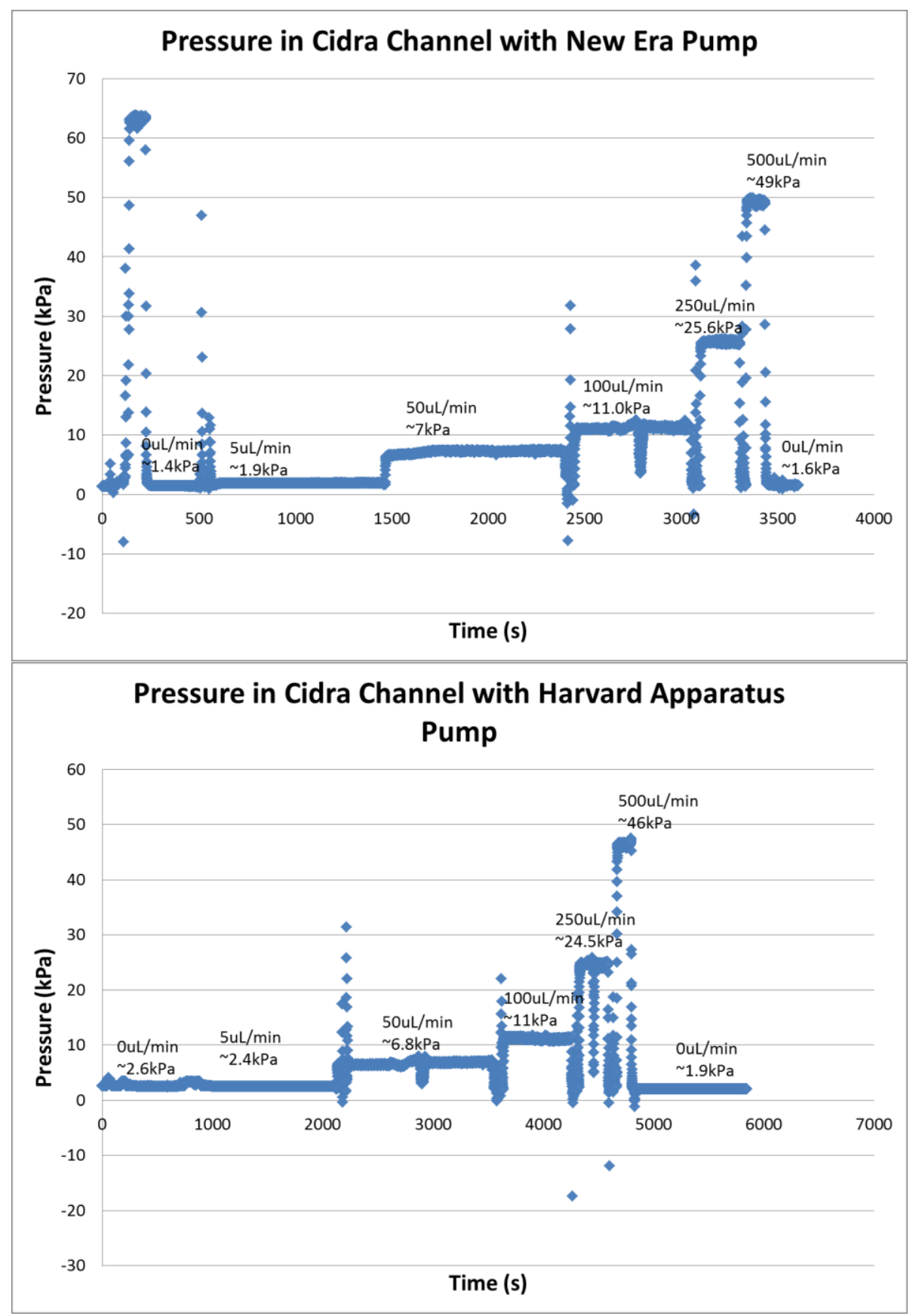

Figure 31. Pressure measurement for Cidra channel. Measurements were taken for both the New Era and Harvard Apparatus syringe pump using a LabSmith pressure sensor. Refilling the syringe caused drops in pressure. 

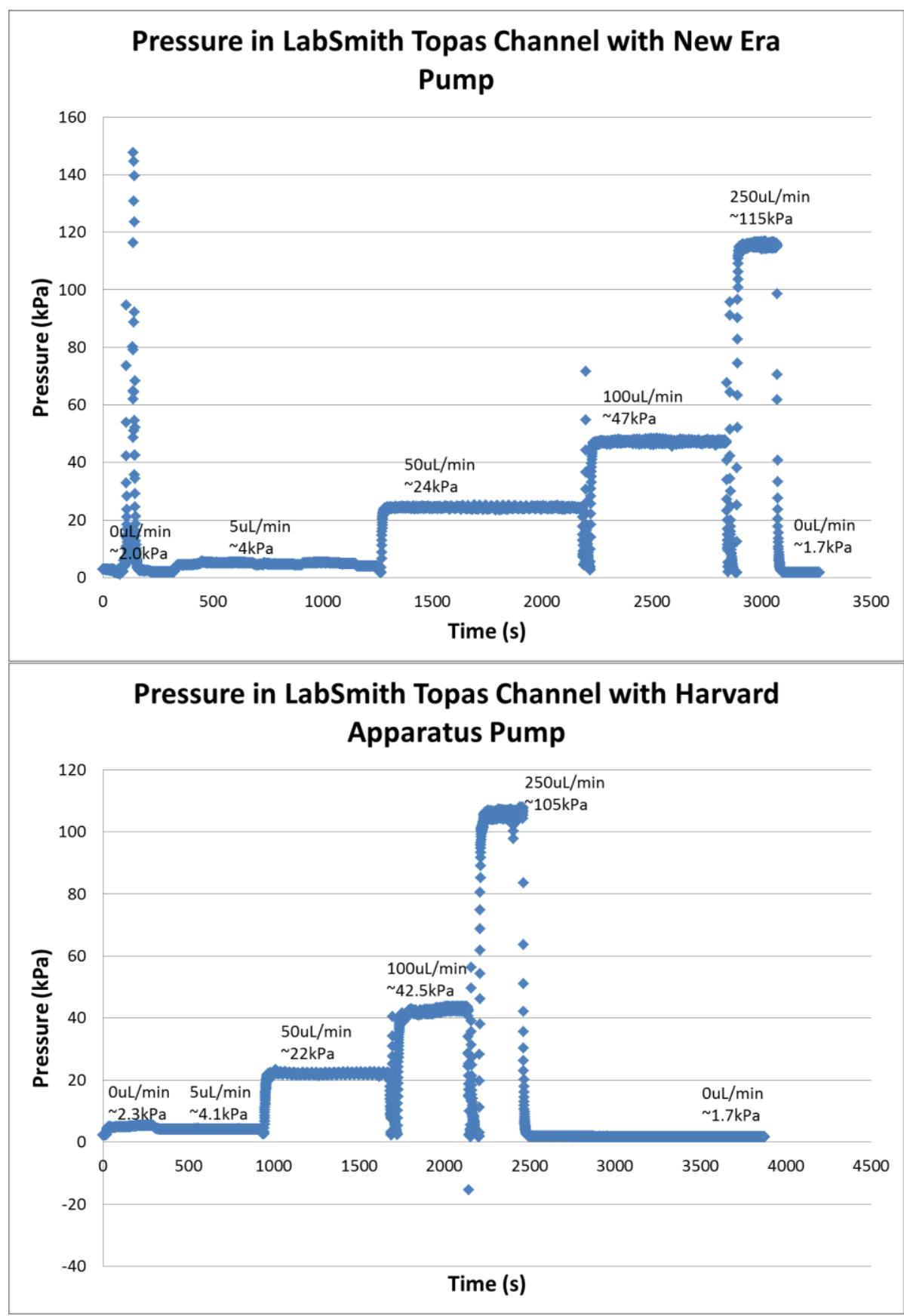

Figure 32. Pressure measurement for LabSmith Topas channel. Measurements were taken by a LabSmith pressure sensor with New Era and Harvard Apparatus syringe pump. The drops in pressure are from refilling the syringe during testing. 

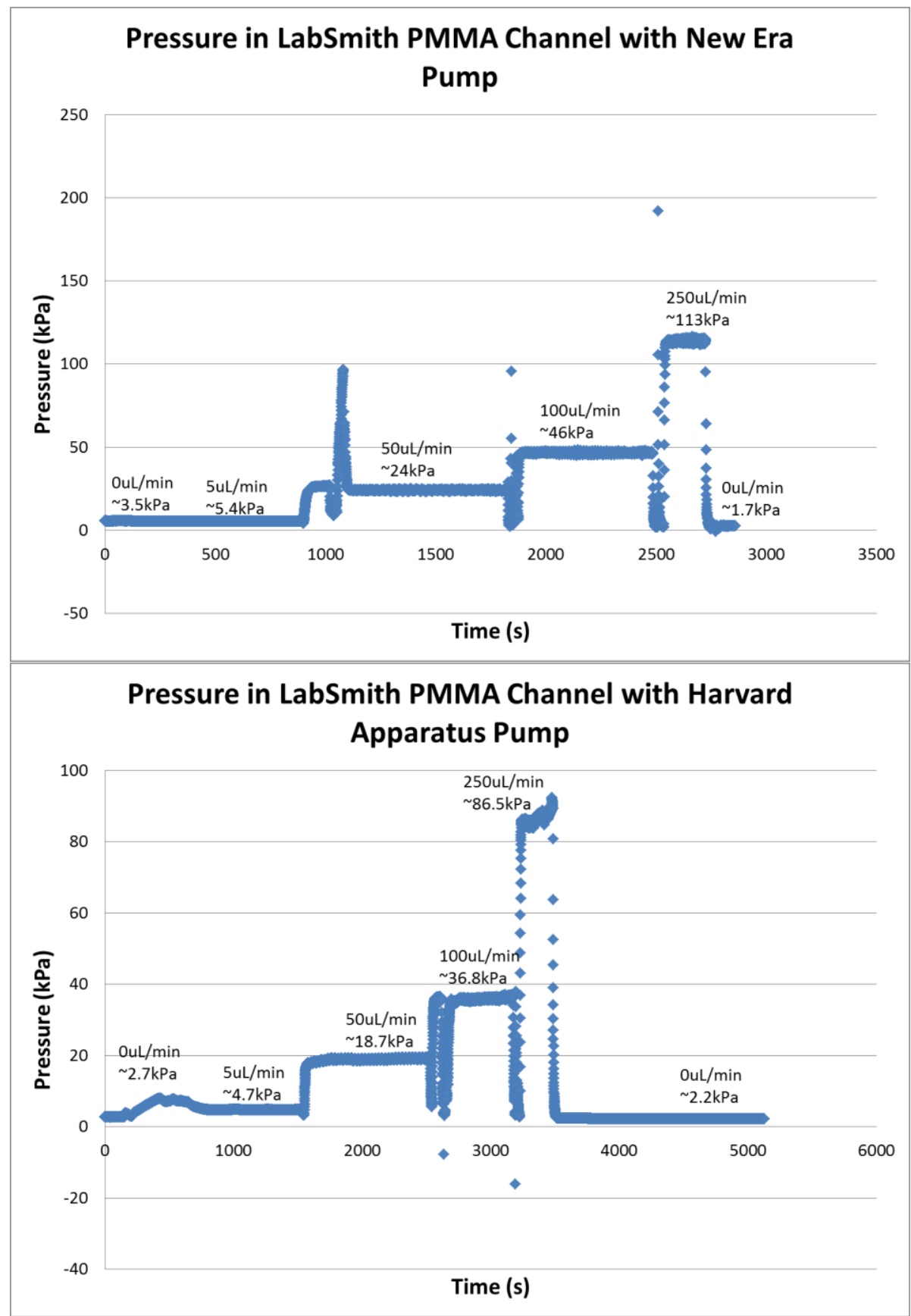

Figure 33. Pressure measurement for LabSmith PMMA channel. Output from LabSmith pressure sensor with the New Era and Harvard Apparatus syringe pumps. The drops during testing are from refilling the syringe.

The average of each flat region was taken as the pressure for that flow rate and compared among the channels (Figure 34, Figure 35). As expected, the LabSmith channels have similar pressures, since they are the same dimensions. The PMMA channel is noticeably different for the Harvard Apparatus Syringe 
Pump. This channel was badly warped and pressure from tape holding the channel to the board might have changed how the fluid flowed through the channel. Other changes could be from a less pulsating and steadier supply of pressure from the syringe pump to the channel.

Just listening to the motors in the pumps, it is easy to hear that there is a difference. The New Era pump motor is heard clicking on and off much more than the Harvard Apparatus pump motor. This did not appear to be making a large difference in the pressures they supply over time, but is believed to have influenced the noise seen in the data and the velocities observed within the channels.

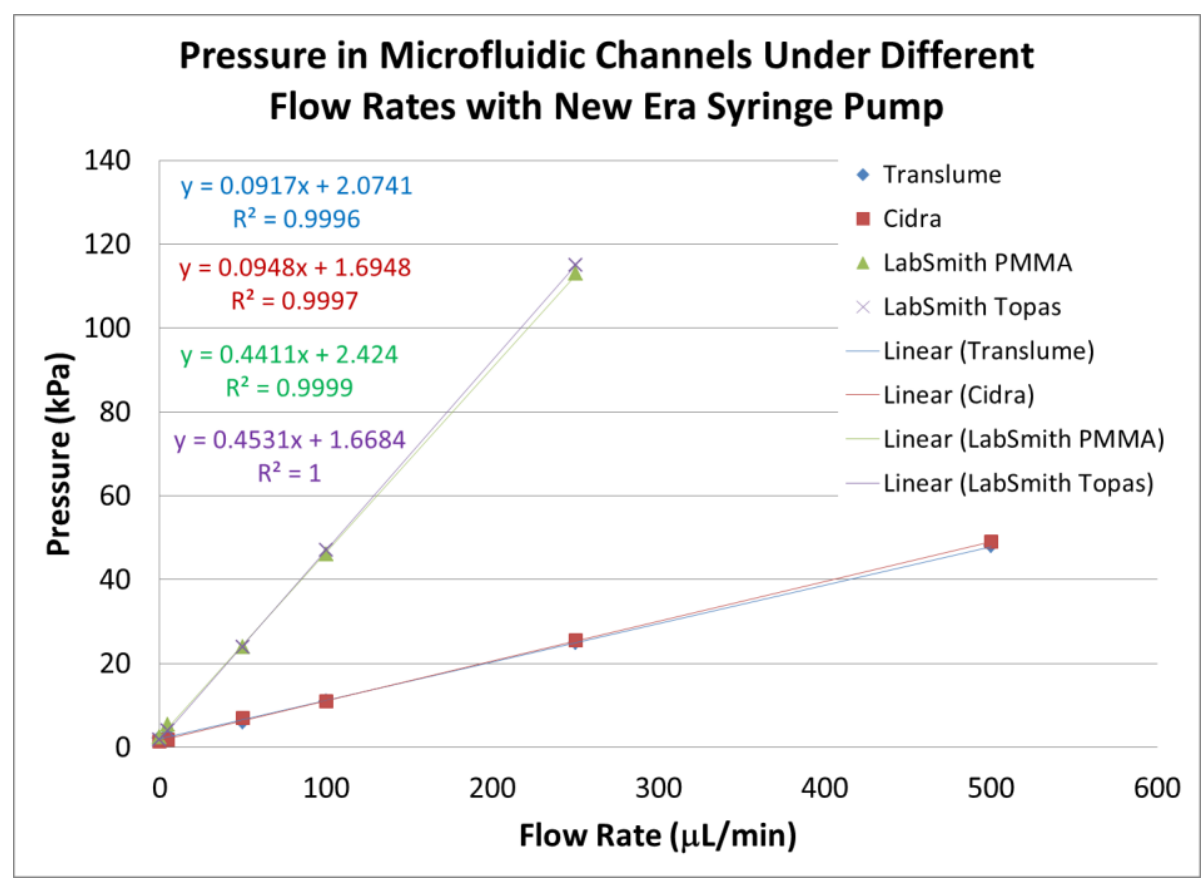

Figure 34. Pressure comparison for channels with New Era syringe pump. Linear models were fit to the measured data to show the linear relationship between pressure and flow rate. 


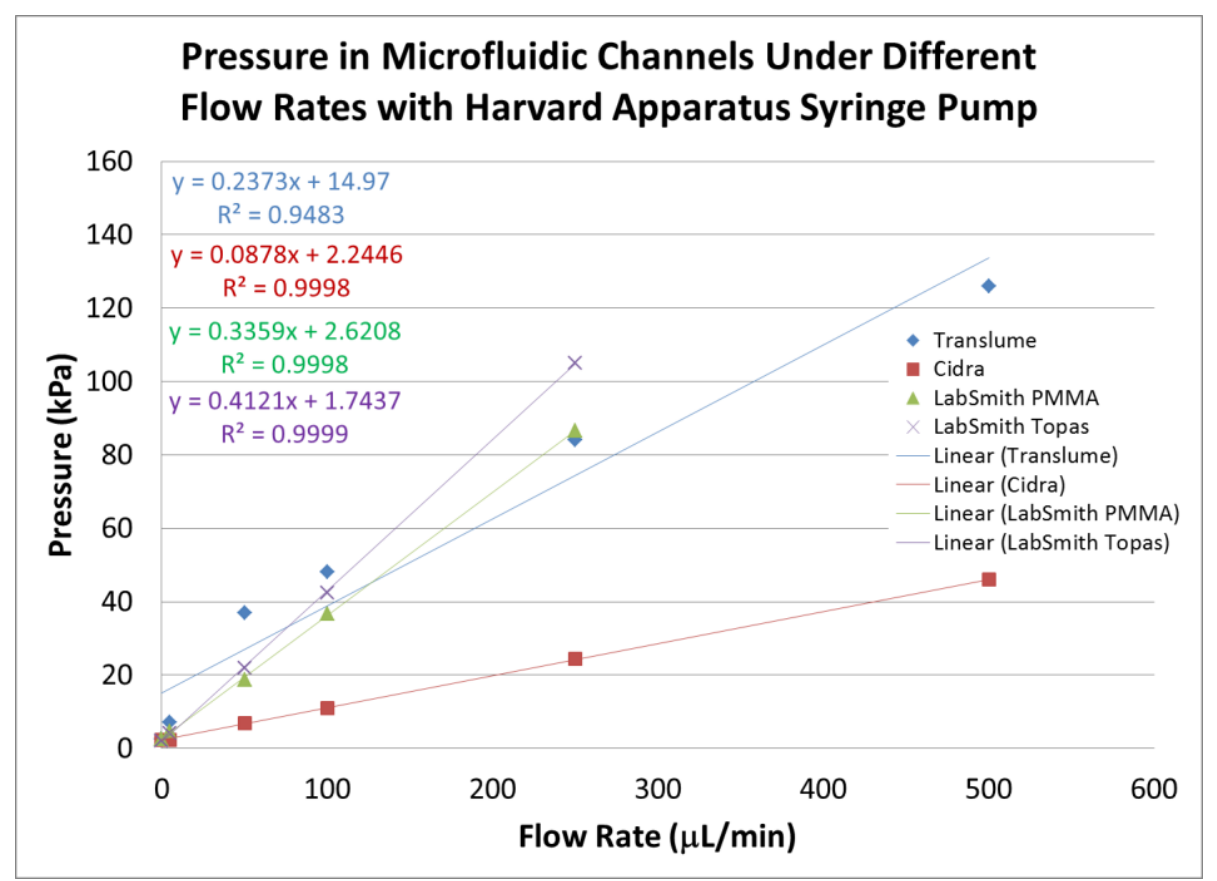

Figure 35. Harvard Apparatus syringe pump channel pressure comparisons. Linear models were fit to the measured data to show the linear relationship between pressure and flow rate.

Theoretical pressures were calculated using Equation 15 [43]. Pressure change $\left(\Delta P_{\mu}\right)$ within microfluidic channels depends on the fluidic resistance $(R)$, the flow rate $(Q)$, the dynamic viscosity $\left(\mu_{d}=1 \times 10^{-3} \mathrm{Ns} / \mathrm{m}^{2}\right.$ for water), the length of the channel $(L)$, the width of the channel $(w)$, and the height of the channel $(h)$.

Equation 15. Pressure change within micro-fluidic channel.

$$
\begin{aligned}
& \Delta P_{\mu}=R \times Q_{\text {flow rate }} \\
& R_{\text {circular channel }}=\frac{8 \mu_{d} L}{\pi r^{4}} \\
& R_{\text {rectangular channel }}=\frac{12 \mu_{d} L}{w h^{3}}
\end{aligned}
$$

The theoretical flow rates were plotted against the measured flow rates for comparison (Figure 36, Figure 37, Figure 38). 


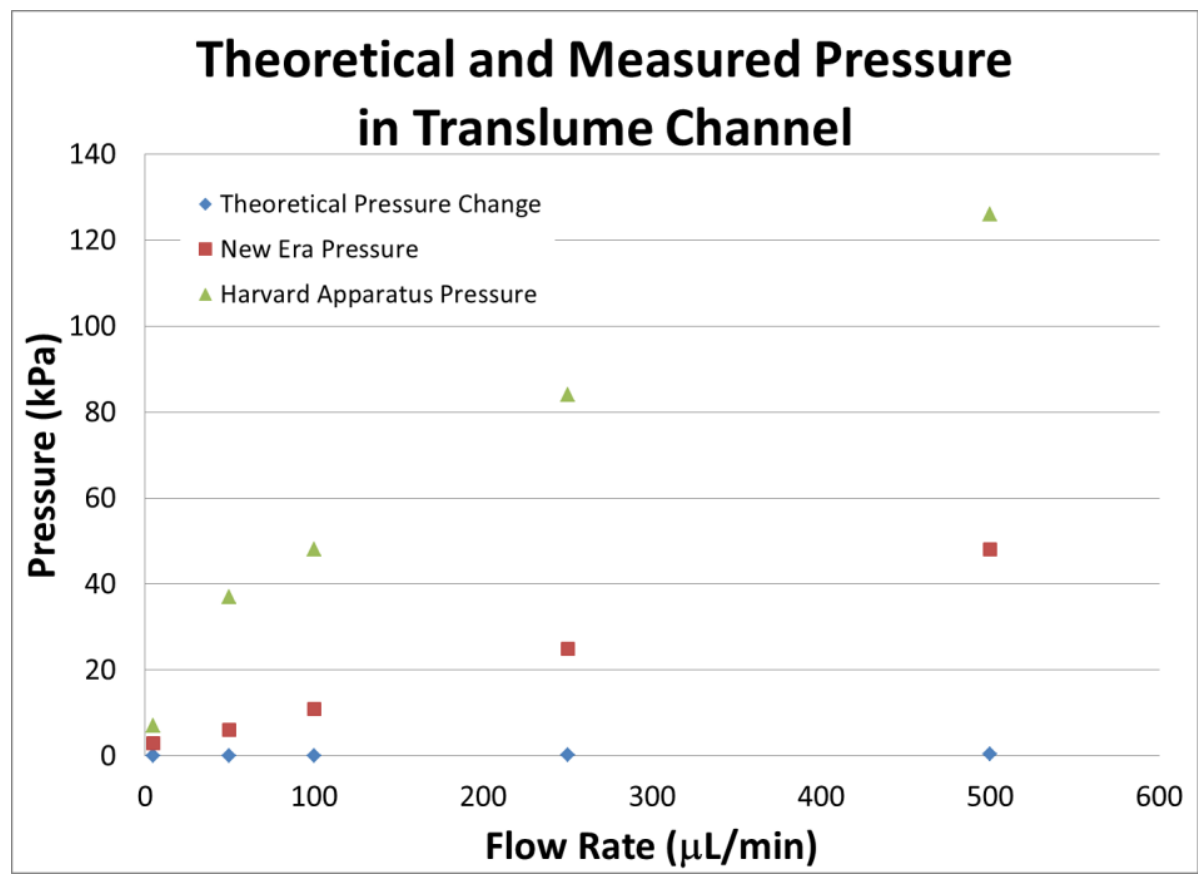

Figure 36. Theoretical and measured pressures for Translume channel.

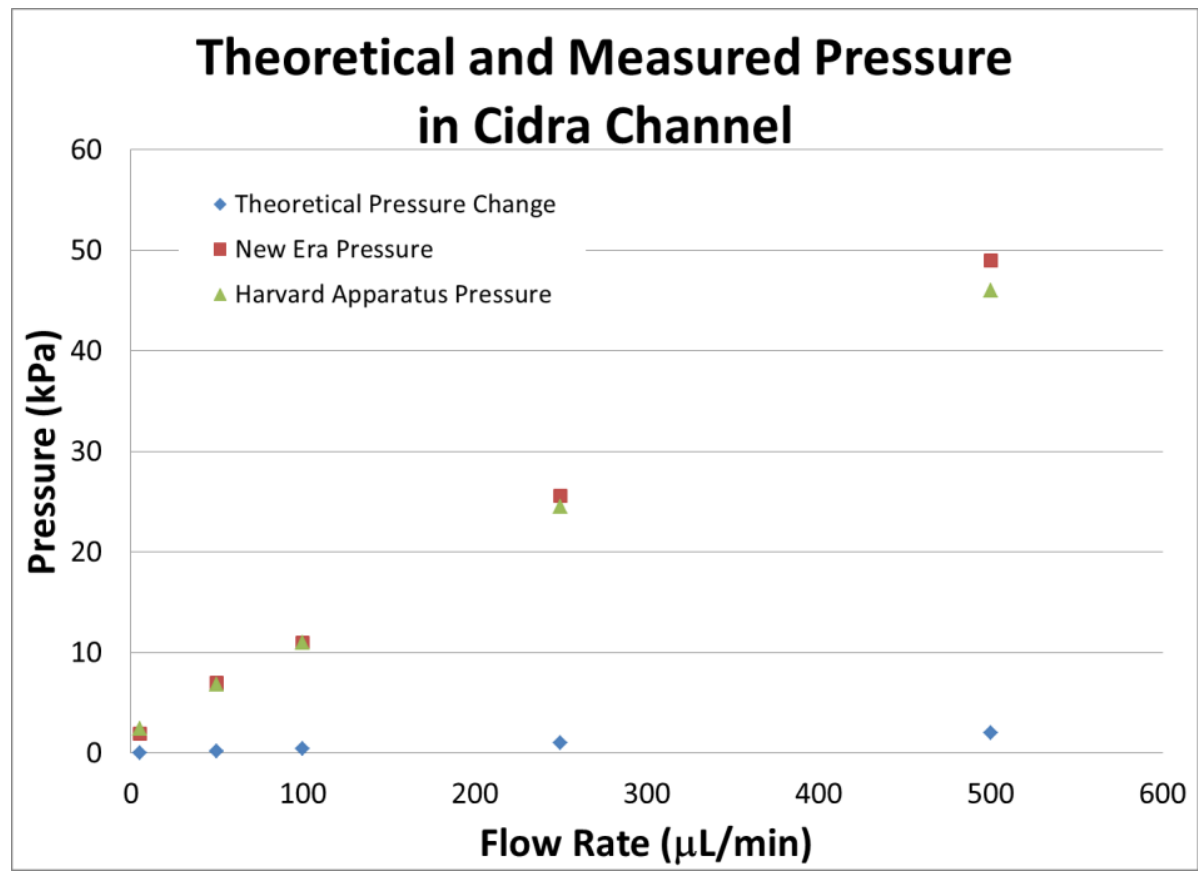

Figure 37. Theoretical and measured pressures for Cidra channel. 


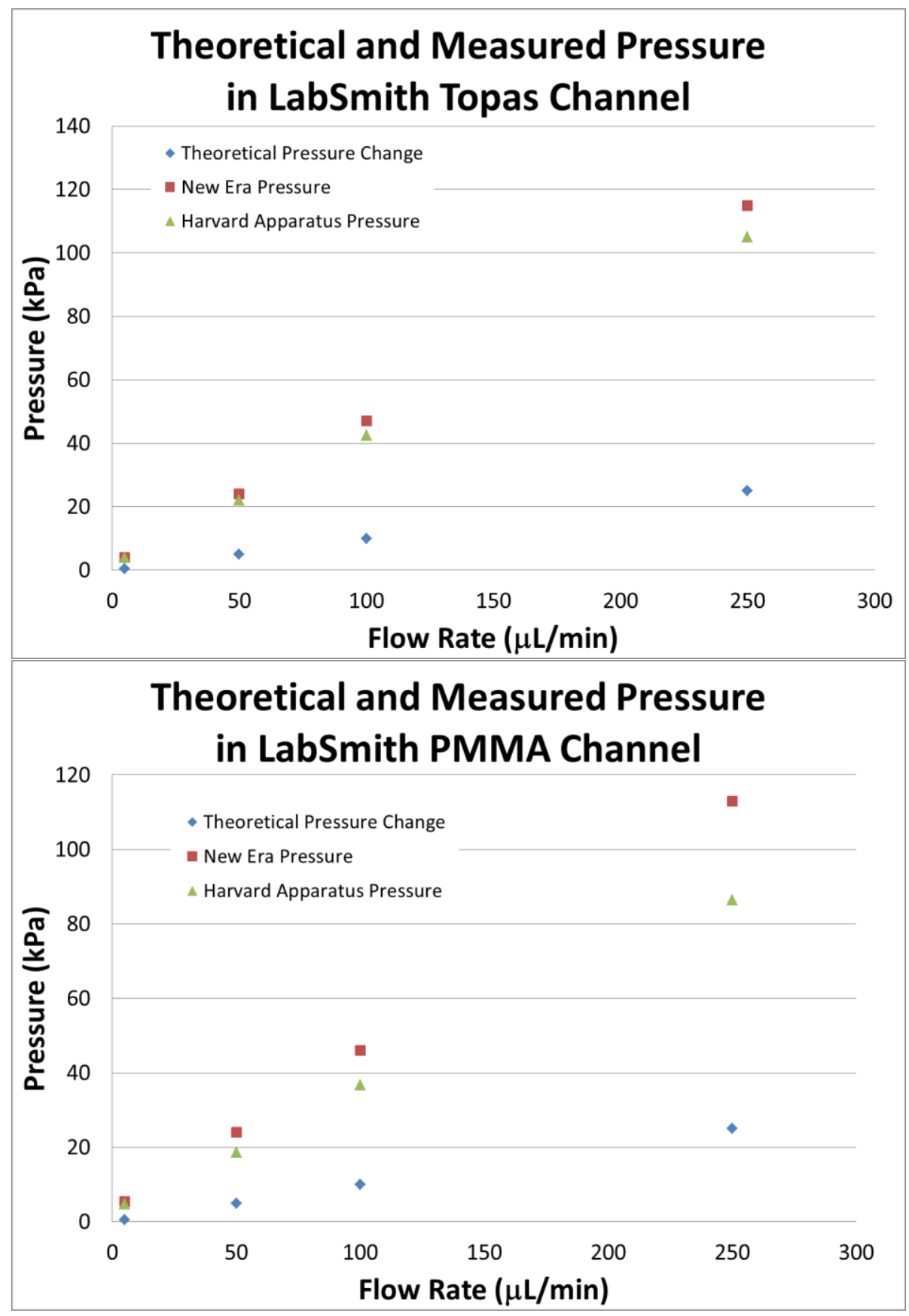

Figure 38. Theoretical and measured pressures for LabSmith channels.

The calculated flow rates are much lower than the measured flow rates, but the measured data fit a linear model very well. These measured flow rates were able to be achieved without any leaking or breaking the channels. It is possible that the measured pressure is higher because of interactions with the tubing not taken into account with these calculations. 


\subsubsection{Measuring Velocity Profiles}

A LabSmith Synchronized Video Microscope was used to obtain particle velocity measurements [26]. This microscope has many different colored LEDs, whose intensities can be adjusted to achieve good contrast of the fluorescent polystyrene particles. With LabSmith's UScope software, probes were placed on a live image of the channel which would measure the velocity of the particles passing through the probe. The output of the probe gives the probe's $x$ - and $y$ position in pixels and the $x$ - and $y$ - velocity of the particles passing through the probe in $\mu \mathrm{m} / \mathrm{s}$. Normally, these probes are squares, but can be elongated to increase signal-to-noise ratio. The channel was aligned on the stage so that the particles flowed in the $y$-direction and the measured $x$-velocity was minimized and considered noise for analysis. Appendix B shows camera and probe settings for the microscope.

Data was received in a .dat file which was converted to a txt file and copied into Excel. This data was reorganized to be compatible with JMP statistical software. In addition, a representation of the probes distance from a wall of the channel was created knowing the number of probes used within the channel's dimensions and a scatterplot of the particle velocity vs. probe position was created. The probe position was converted into an approximate distance from the wall by dividing the width across which the probes were spaced by twice the number of probes used (Figure 39). This gave the distance of the center of the first probe from the wall, and half the distance of the center of one probe to the center of the 
next probe. It was assumed that the probes were equally spaced across the channel width.

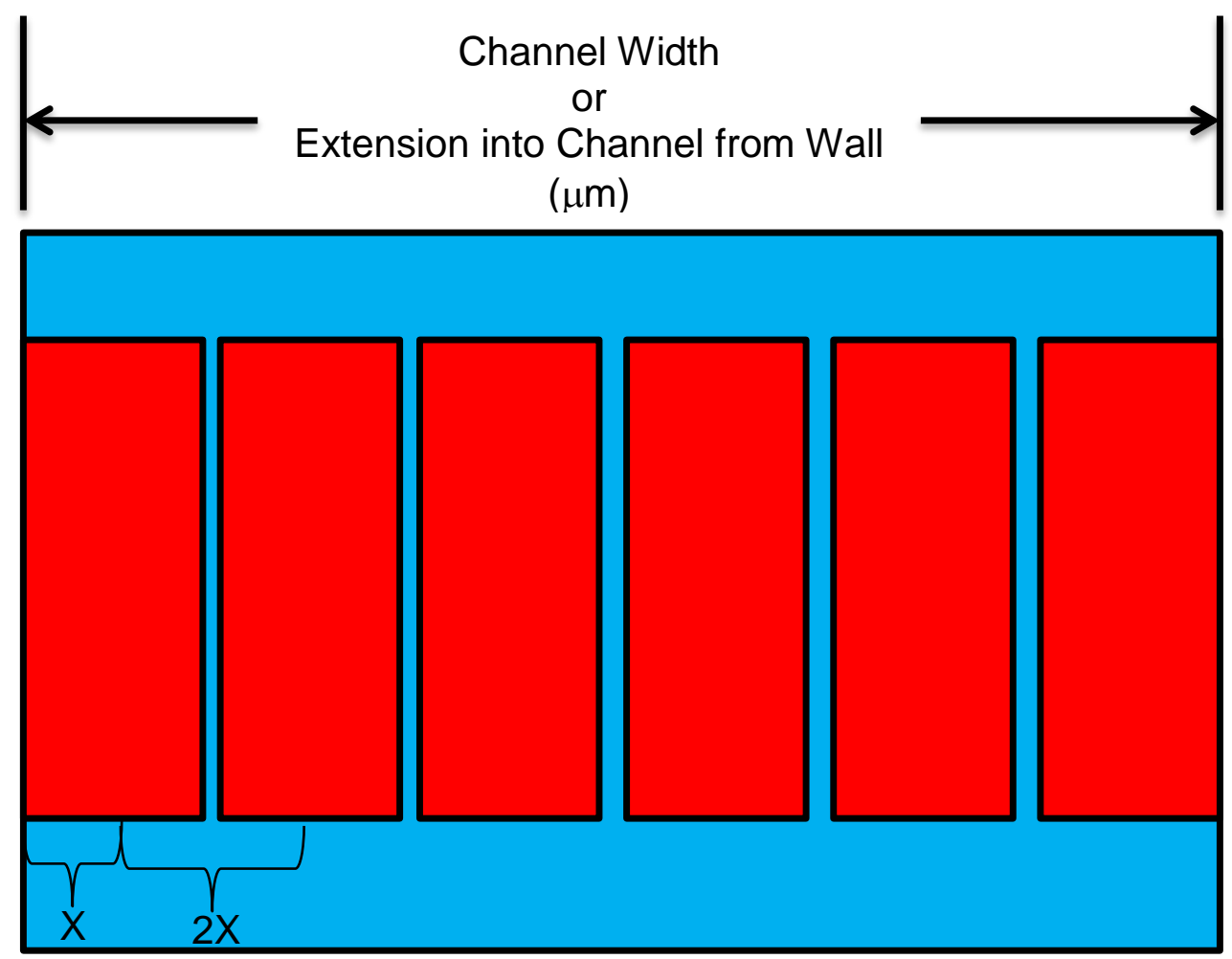

Figure 39. Representation of probe position conversion.

The data was then copied into JMP statistical software. Plots were made showing the mean and standard error at each probe position. There is some noise for each probe but most of the data points are in the same general region. It is expected that the noise is from imaging noise that the LabSmith software mistakenly identified as a particle or particles that were moving too fast for the software to measure accurately. 


\subsection{ELECTRO-KINETIC DRIVEN FloW EXPERIMENTAL PROCEDURES}

\subsubsection{Applying Voltage}

A LabSmith High Voltage Sequencer (S/N\#: H3000R182 0109) was used to apply a voltage to the channel. Appendix $C$ shows settings used for the sequencer. Two pieces of 3 to 5 inch copper wire with plastic were stripped at both ends for about $3 / 4$ to 1 inch. One end was wrapped around an 18 gage syringe tip and the other end was inserted into the HCV High Voltage Cable connecting the Voltage Sequencer. The syringe tip was placed into the inlet and outlet connector of the channel (Figure 40). The syringe tip was used because stainless steel would not corrode like copper would in the channel itself but still be able to carry the voltage.

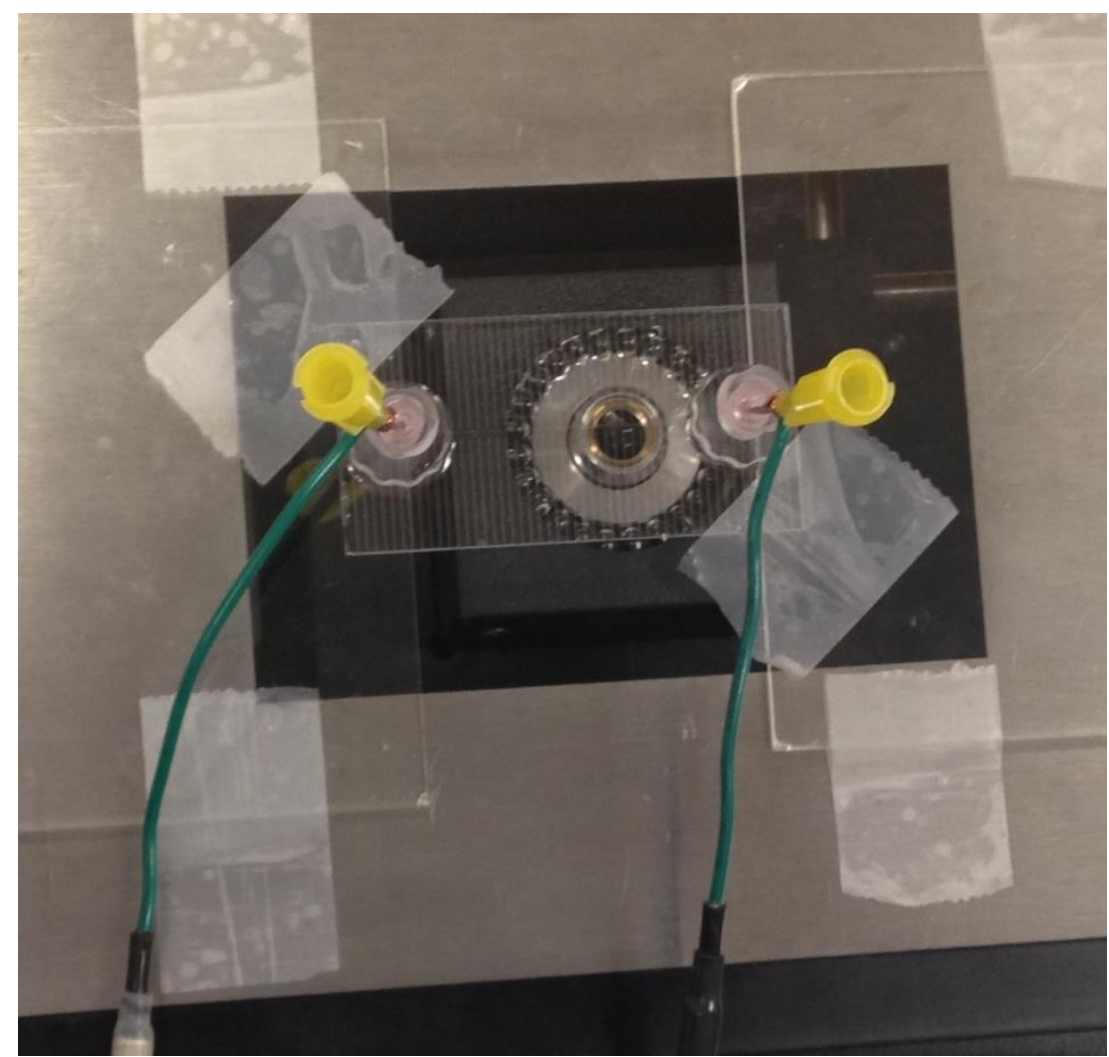

Figure 40. Setup of syringe tips and copper wire in channel inlet/outlet. 


\subsubsection{Measuring Voltage}

Voltage was measured to ensure that the Sequencer output was comparable to its setting. The voltage was measured with a Fluke handheld multi-meter and a second channel of the LabSmith Sequencer. The measurements showed a slightly lower voltage than the Sequencer was set to deliver for both measurement devices; however, this could be from internal resistance of the measurement devices (Figure 41). Both the measurement devices measured nearly the exact same voltage output for each voltage setting and these measurements have a very linear trend which would be expected from a resistance causing the voltage drop.

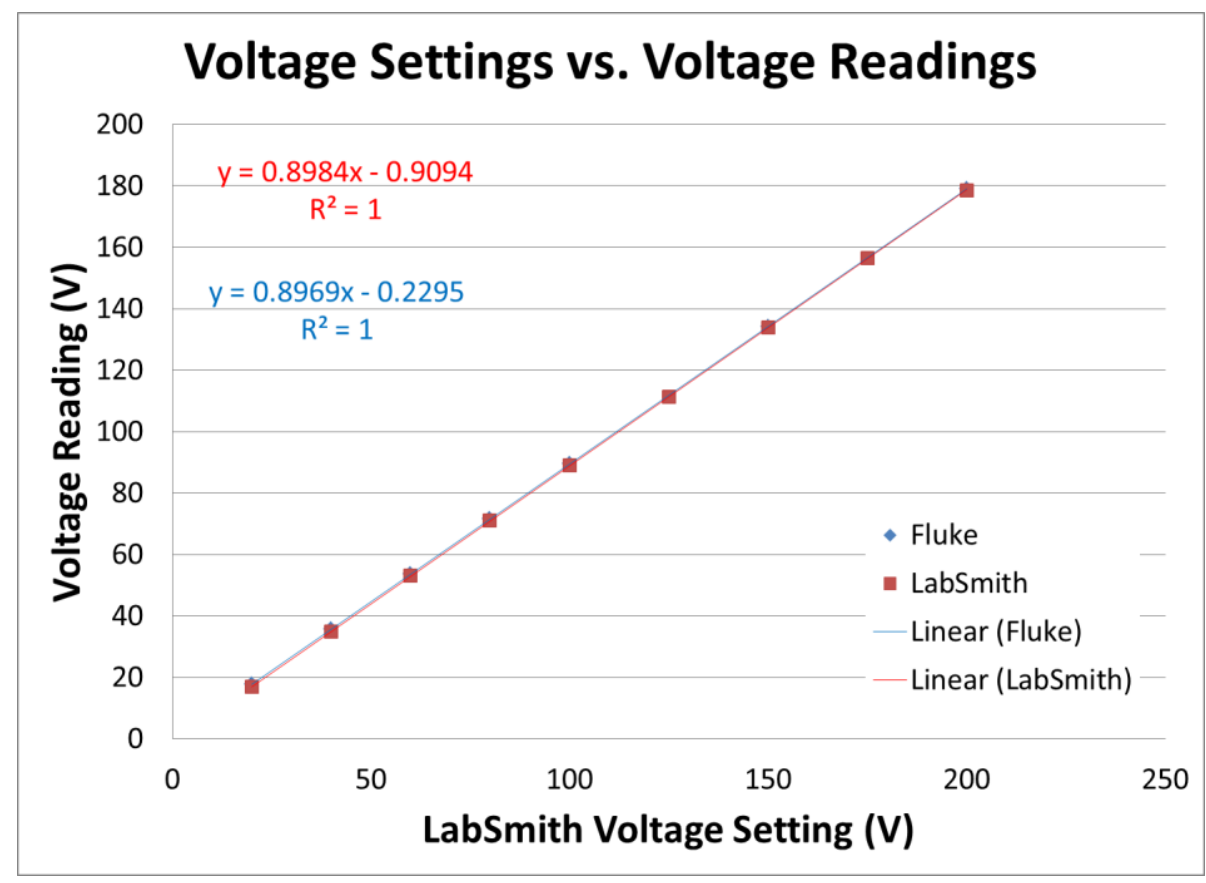

Figure 41. Measured voltage from LabSmith High Voltage Sequencer.

\subsubsection{Measuring Velocity Profiles}

The same setup of the LabSmith Video Microscope was used as for the pressure driven flows. To prepare the channel, a small volume of the particles solution was 
placed in each inlet/outlet. This was then allowed to rest for 1 hour with the microscope light off to allow pressure to equalize within the channel and reduce its influence on the electro-kinetic measurements. The voltage was applied for approximately 30 seconds before measurement readings were taken to allow the particles to obtain their highest velocities possible. Measurement readings were taken for approximately 30 seconds to prevent pressure build up from slowing the particles and influencing the measurements. The voltage was then set back to $0 \mathrm{~V}$ for 15 minutes before the next voltage was tested. The data files were reorganized in Excel and analyzed in JMP as with the pressure driven flows. 
Chapter 5 Results, Analysis, and Discussion

\subsection{Pressure-DRIVEN FLOW}

Pressure-driven flow was measured through each of the 4 available channels at varying flow rates to determine the effect of channel dimension on velocity measurements.

\subsubsection{Results and Analysis}

\section{Translume Channel}

The Translume channel was measured at multiple flow rates ranging from $1 \mu \mathrm{L} / \mathrm{min}$ to $5 \mu \mathrm{L} / \mathrm{min}$ with a probe size of 64 pixels by 256 pixels $(42.7 \mu \mathrm{m}$ by $163.7 \mu \mathrm{m})$ using $1 \mu \mathrm{m}$ diameter fluorescent polystyrene spheres. A 10x magnification objective was used for the LabSmith Syncronized Video Microscope (Figure 42).

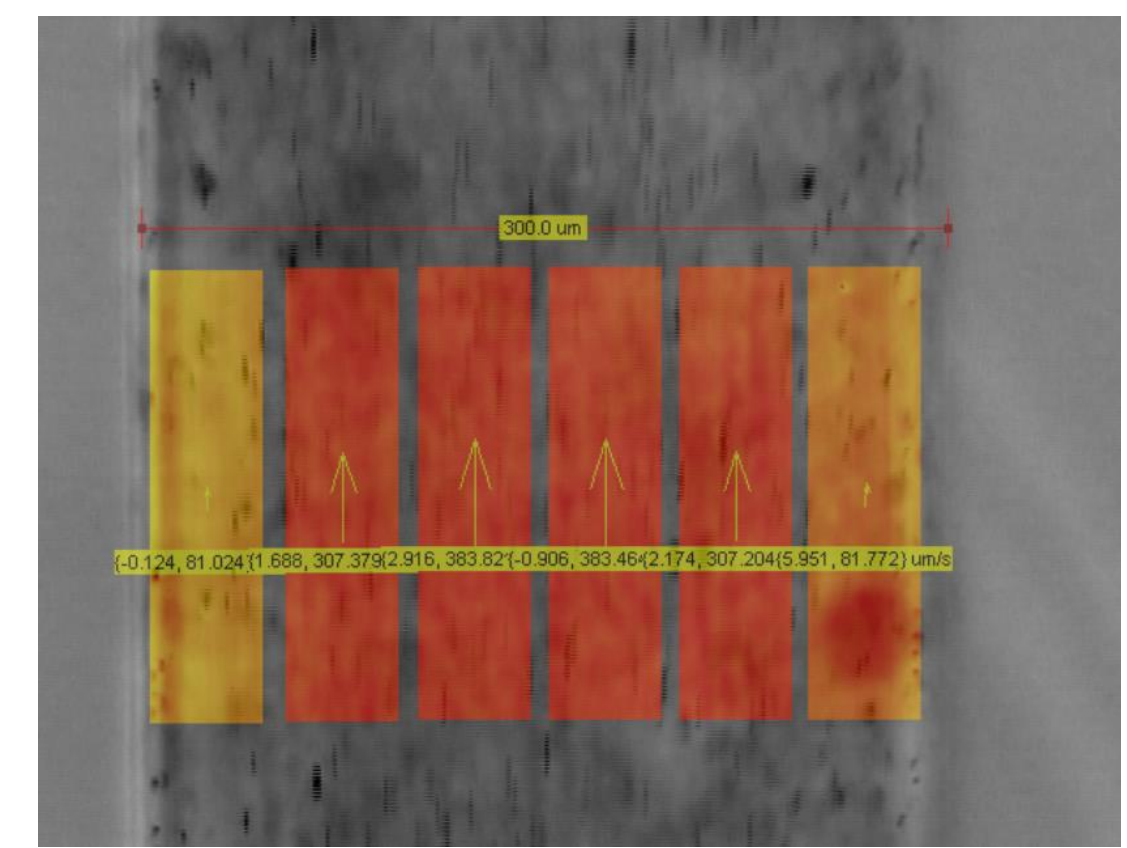

Figure 42. Probe setup within Translume channel. Setup of probes within Translume channel. The probes were evenly spaced across the channel with the best size option to measure the particle velocities. 
The center probes for the $5 \mu \mathrm{L} / \mathrm{min}$ data with the New Era syringe pump was removed from the overall dataset because the measured velocities in the center were much lower than near the walls indicating the particles were moving too fast for the software to measure accurately. Overall, observing the means and standard errors, the data appears to have a parabolic profile (Figure 43, Figure 44, Figure 45, Figure 46).

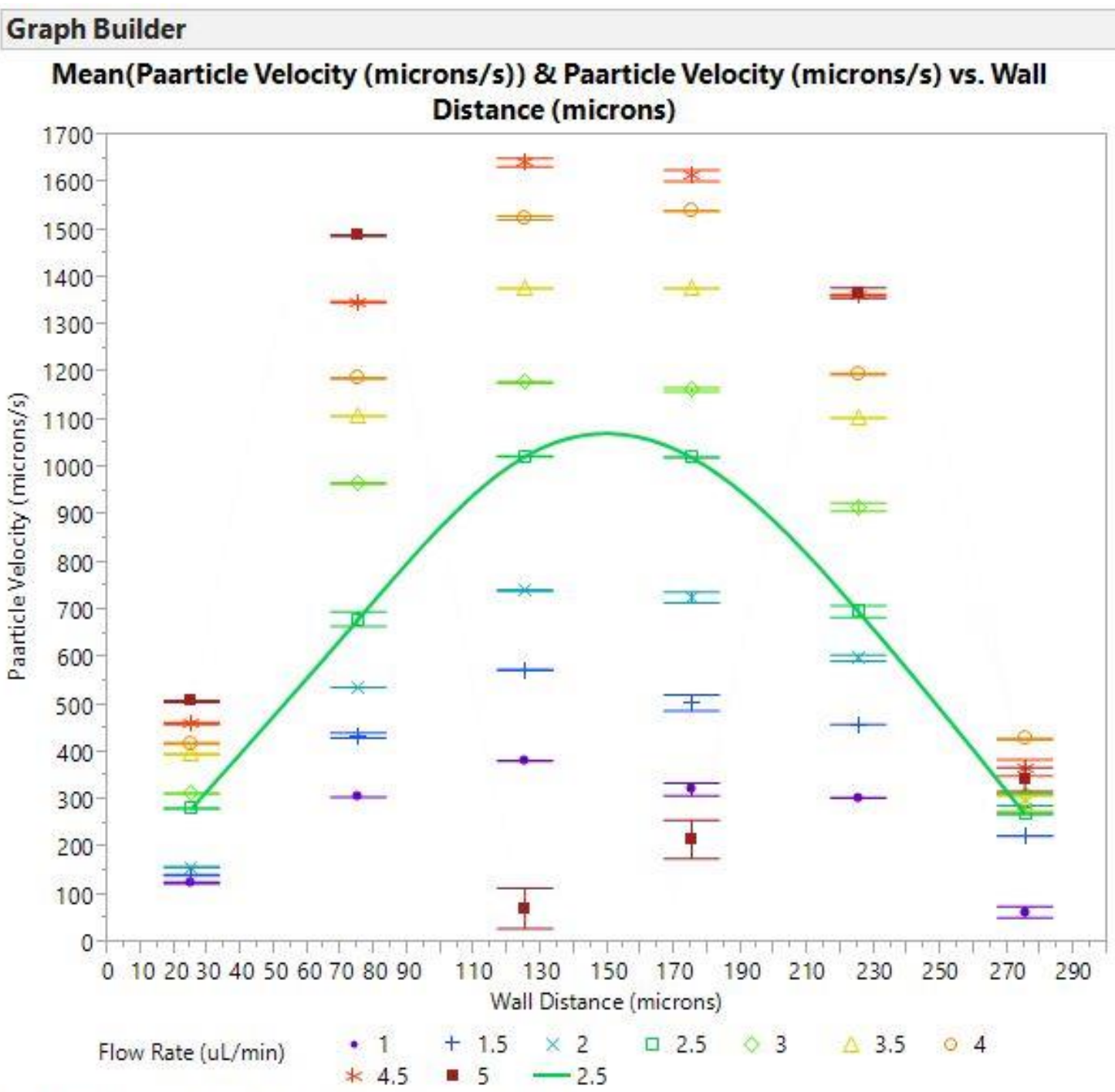

Each error bar is constructed using 1 standard error from the mean.

Figure 43. Graph for Translume channel with New Era syringe pump. 


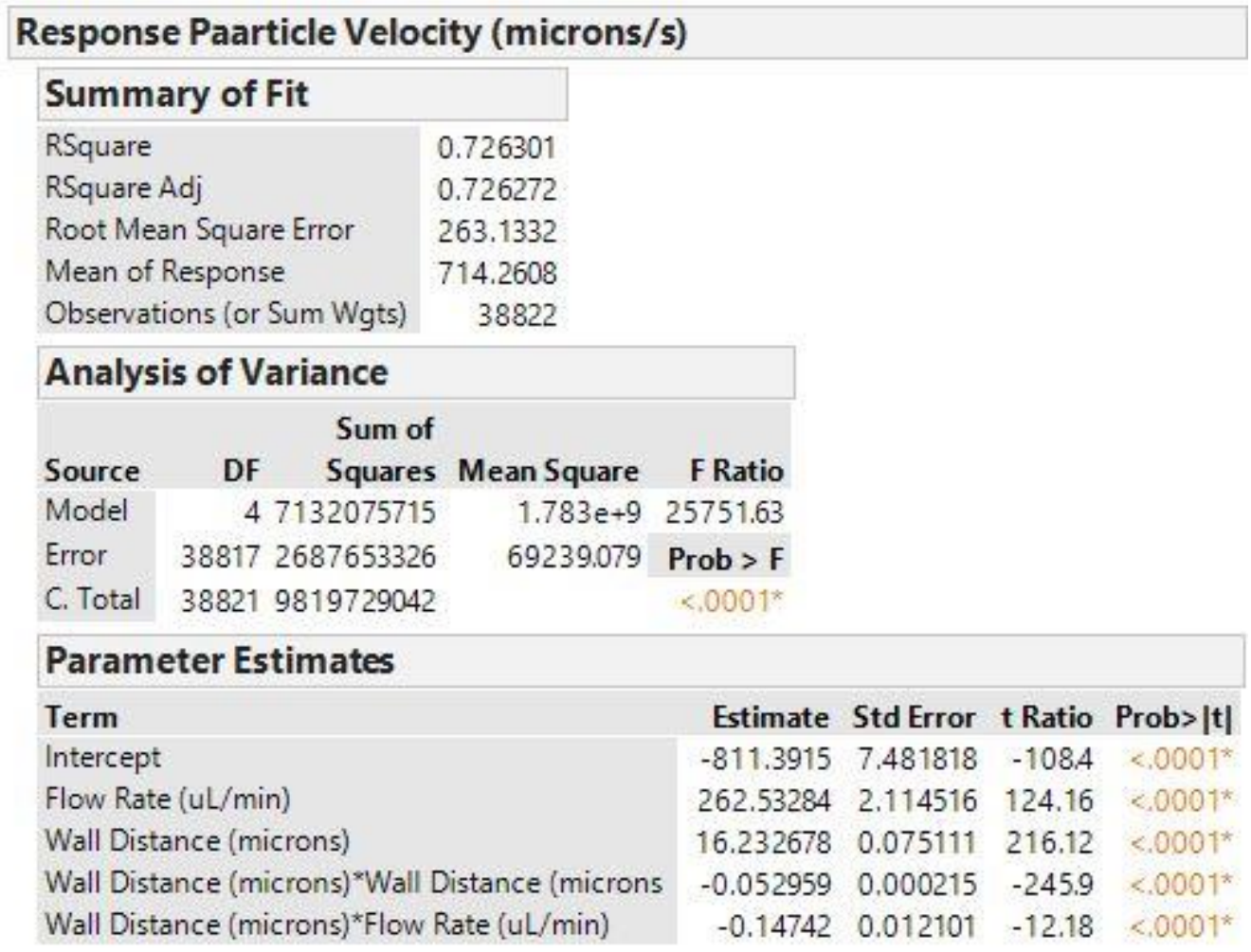

Figure 44. JMP output for model of Translume channel with New Era syringe pump. 


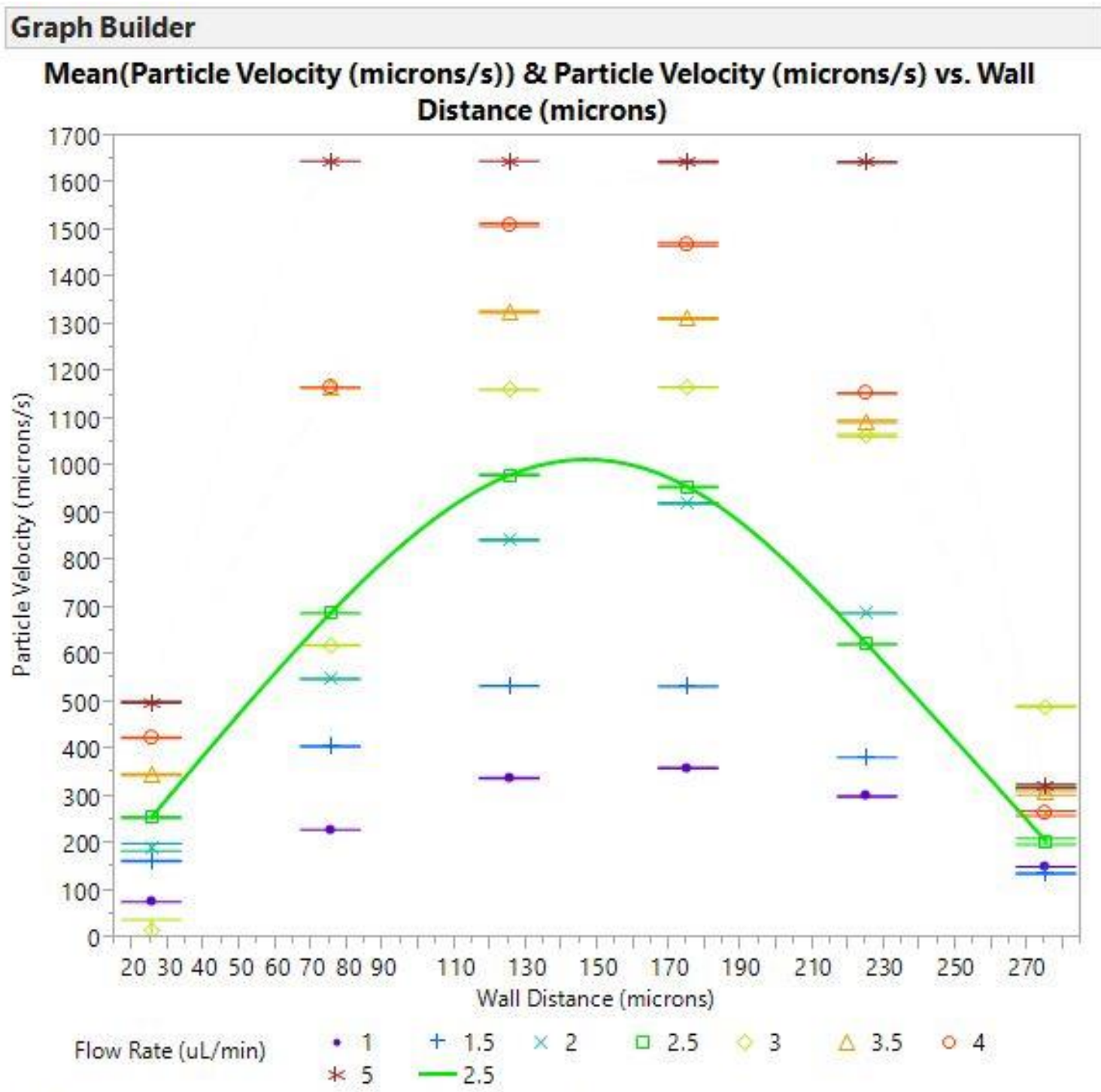

Each error bar is constructed using 1 standard error from the mean.

Figure 45. Graph of Translume channel with Harvard Apparatus syringe pump. 


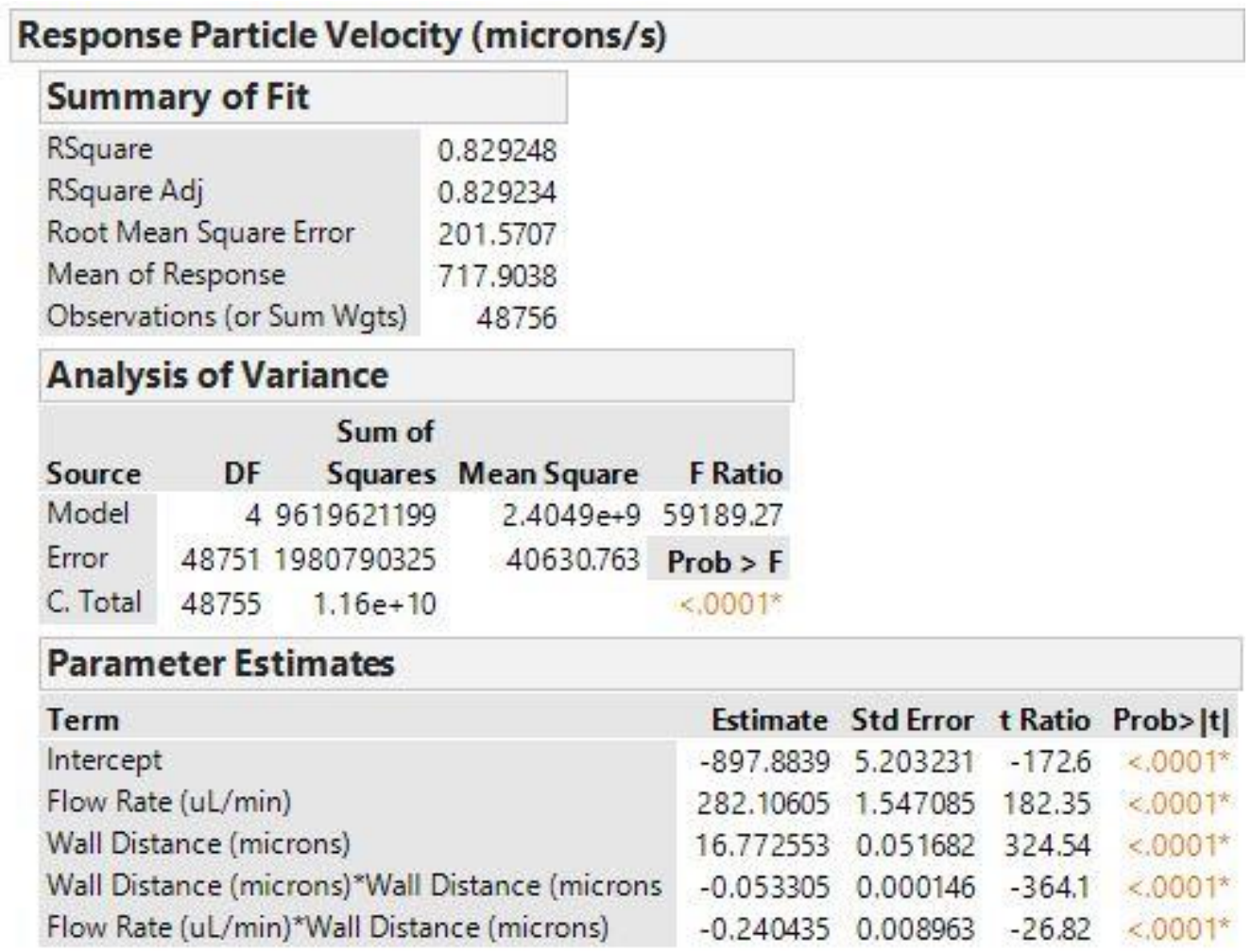

Figure 46. JMP output for model for Translume channel with Harvard Apparatus syringe pump. The distance from the wall and the square of the distance from the wall are both significant to the velocity. In addition, there appears to be a different effect of the position depending on the flow rate. The velocities measured at a given flow rate are fairly similar for each syringe pump but there are differences, possibly due to pulsing. The parabolic profile is very significant at all flow rates with both syringe pumps. Also, the flow rates seem to be evenly distributed from each other.

\section{Cidra Channel}

The Cidra channel was measured at multiple flow rates ranging from $2.5 \mu \mathrm{L} / \mathrm{min}$ to $15 \mu \mathrm{L} / \mathrm{min}$ with a probe size of 64 pixels by 256 pixels $(21.3 \mu \mathrm{m}$ by $82.2 \mu \mathrm{m})$ using $2 \mu \mathrm{m}$ diameter fluorescent polystyrene spheres. A 20x magnification objective was used for the LabSmith Syncronized Video Microscope (Figure 47). 
Originally, the $4 x$ magnification objective and 10x magnification objective was used to obtain as much of the width of the channel as possible, but no velocity difference was seen along the channel.

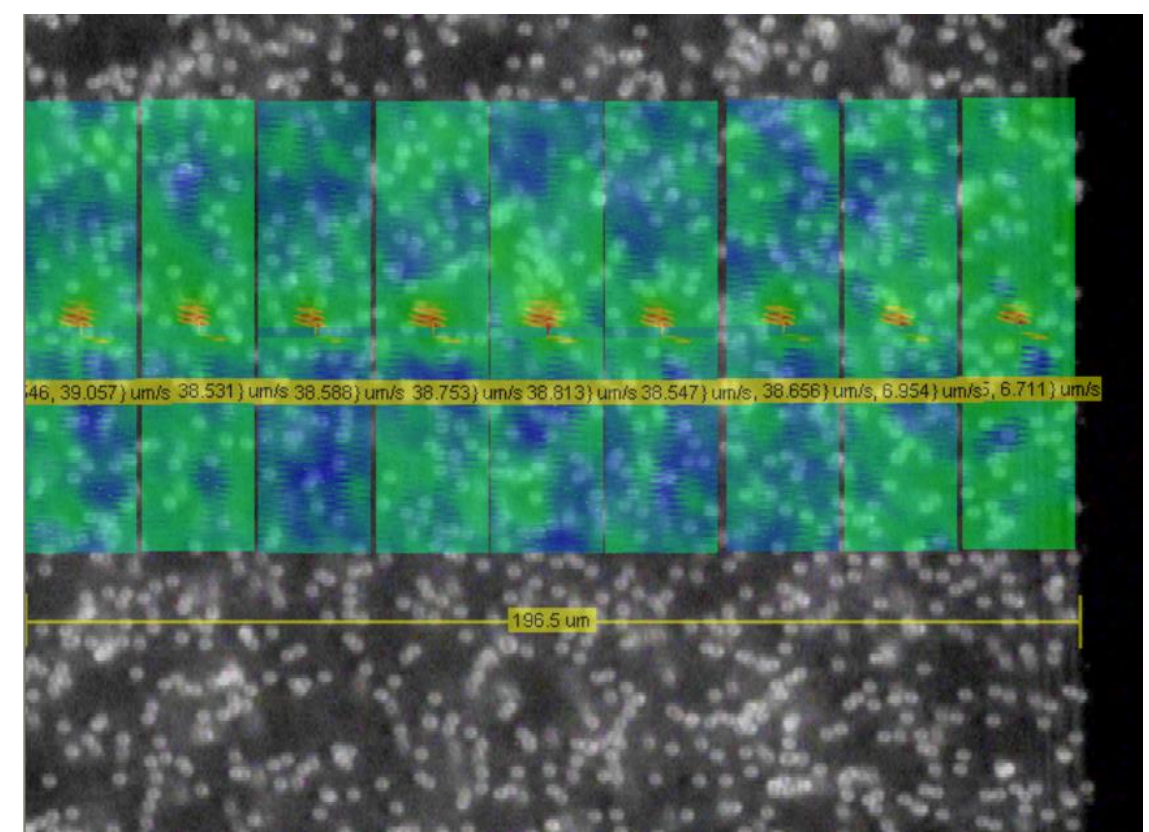

Figure 47. Setup of probes within Cidra channel for New Era syringe pump.

Since this channel is very wide and shallow, the parabolic profile is not really seen except for right next to the wall (Figure 48, Figure 49). Different velocity ranges were measured at different magnification objectives. 


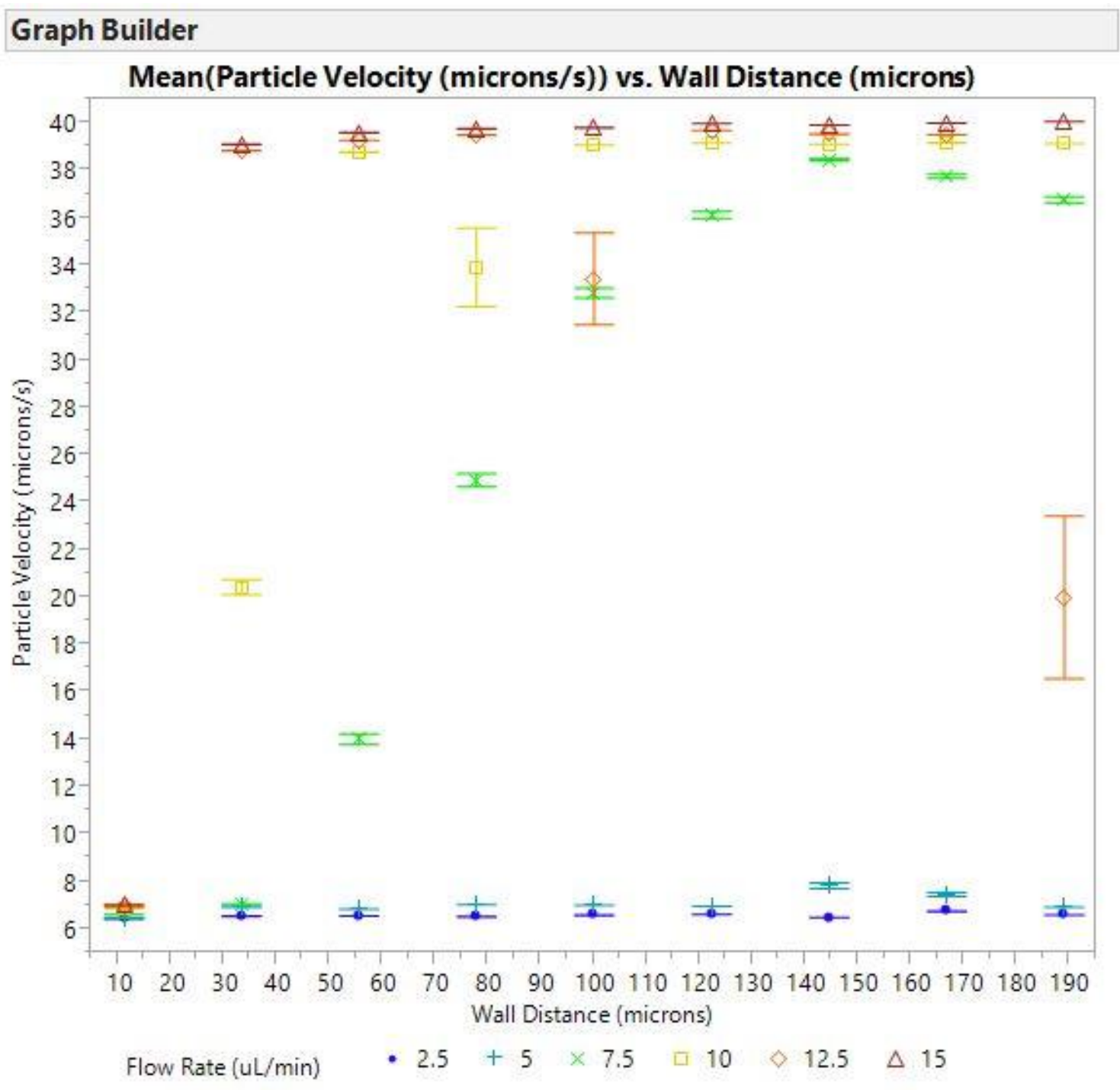

Each error bar is constructed using 1 standard error from the mean.

Figure 48. Graph of Cidra channel with New Era syringe pump. 


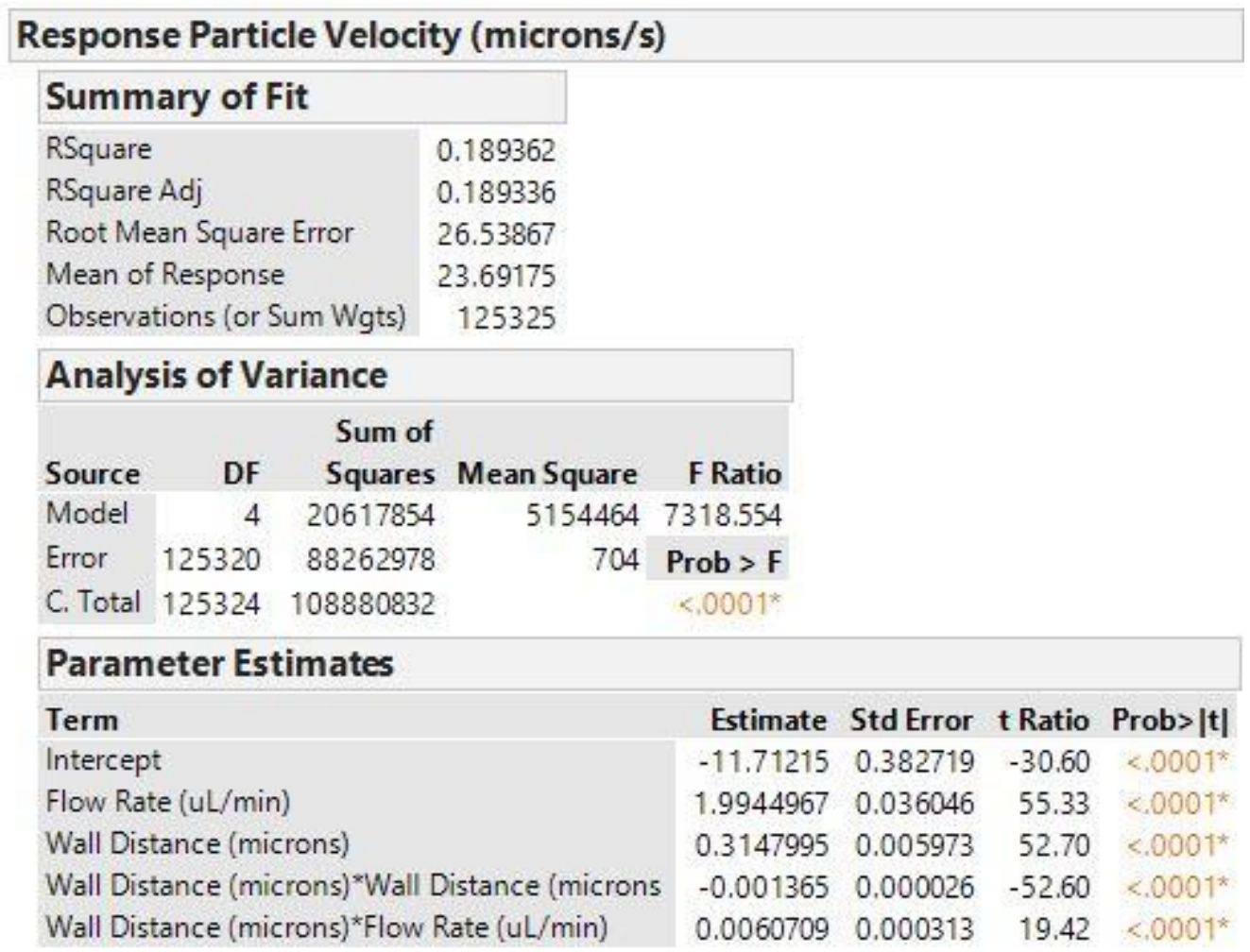

Figure 49. JMP output for model for Cidra channel with New Era syringe pump.

The channel was re-measured with the Harvard Apparatus Syringe Pump to determine if a better profile would be observed. The same flow rates and probe sizes were used but with a $1 \mu \mathrm{m}$ PS bead solution using the $20 \mathrm{x}$ magnification objective (Figure 50). 


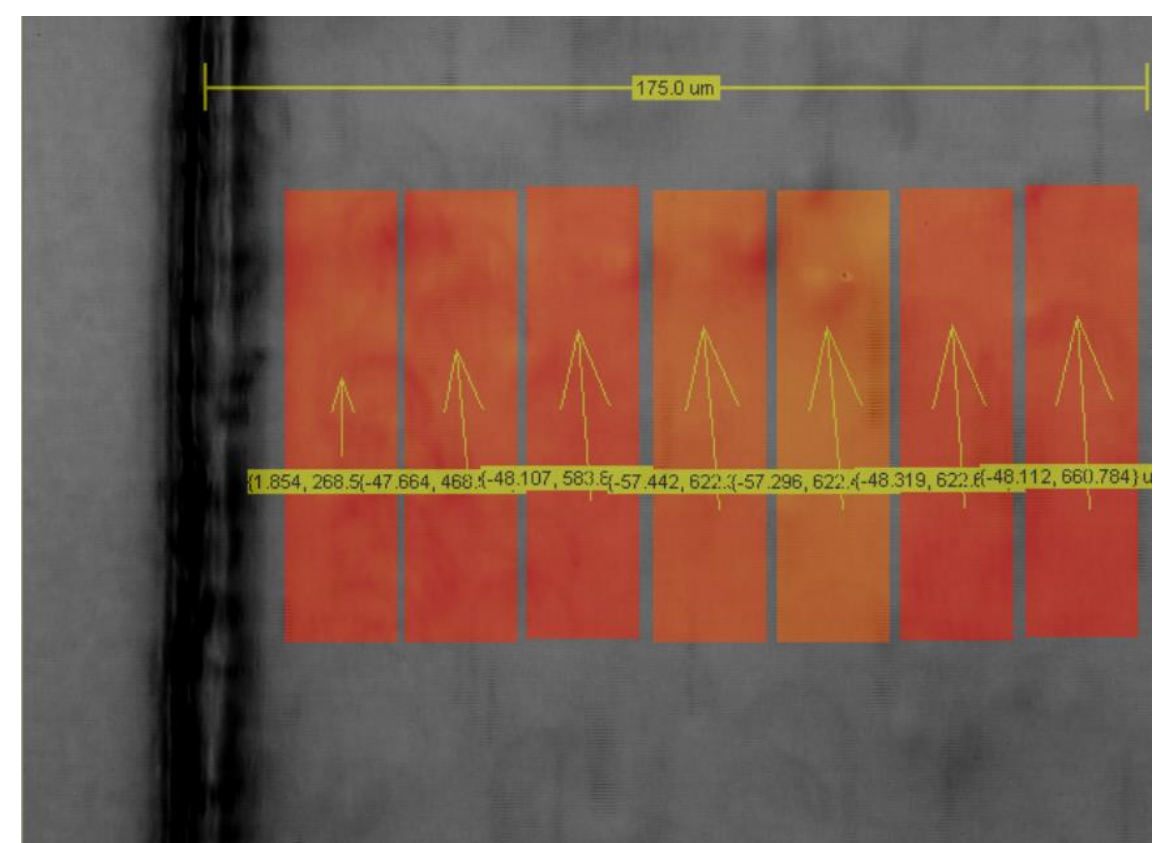

Figure 50. Setup of probes within Cidra channel for Harvard Apparatus syringe pump.

The data for the Harvard Apparatus Syringe Pump still does not show a parabolic profile (Figure 51, Figure 52). It is expected that the pancake shape of the channel makes it much more difficult to obtain a middle plane of the channel and leads to non-reproducible results. Although these channels are easy to fabricate in the lab, they are not ideal for measurement purposes. 


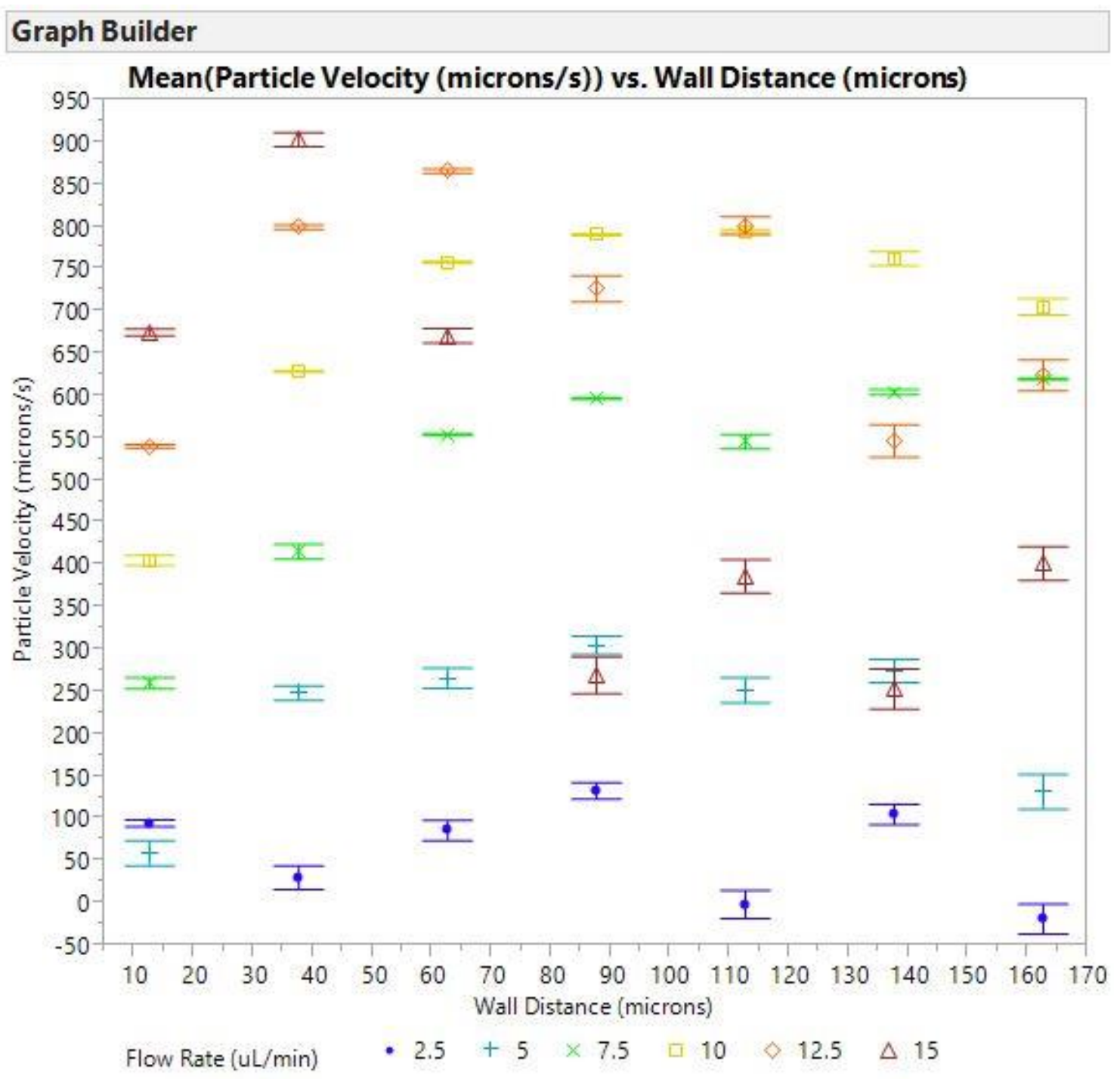

Each error bar is constructed using 1 standard error from the mean.

Figure 51. Graph of Cidra channel with Harvard Apparatus syringe pump. 


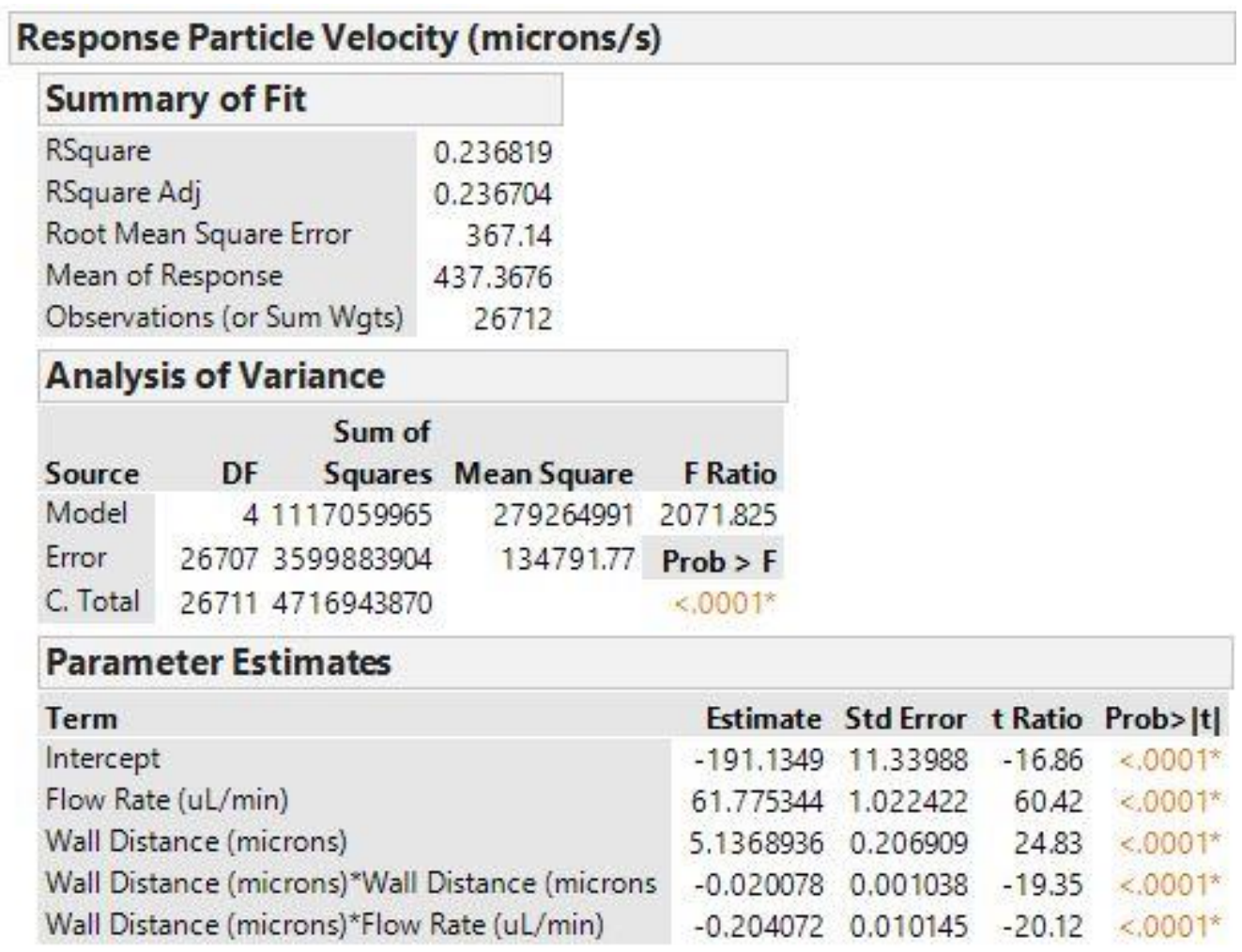

Figure 52. JMP output for model for Cidra channel with Harvard Apparatus syringe pump.

\section{LabSmith Topas Channel}

The LabSmith Topas channel was measured at multiple flow rates ranging from

$0.1 \mu \mathrm{L} / \mathrm{min}$ to $2 \mu \mathrm{L} / \mathrm{min}$ with a probe size of 64 pixels by 256 pixels $(21.3 \mu \mathrm{m}$ by

$82.2 \mu \mathrm{m})$ using $2 \mu \mathrm{m}$ diameter fluorescent polystyrene spheres. A 20x

magnification objective was used for the LabSmith Syncronized Video

Microscope (Figure 53). 


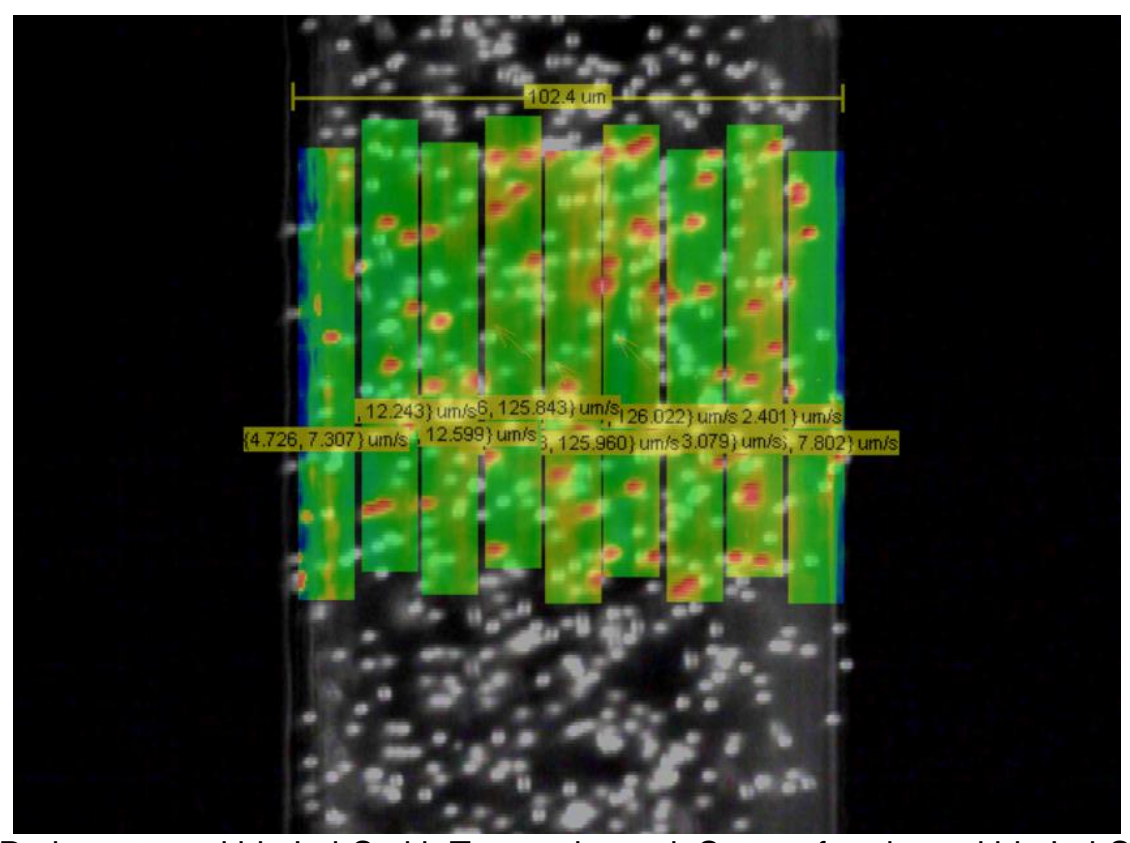

Figure 53. Probe setup within LabSmith Topas channel. Setup of probes within LabSmith Topas channel for New Era Syringe Pump. The probes were evenly spaced across the channel with the best size option to measure the particle velocities.

No pattern was seen in the data, most likely because the syringe pump pulses at very low flow rates (Figure 54, Figure 55). 


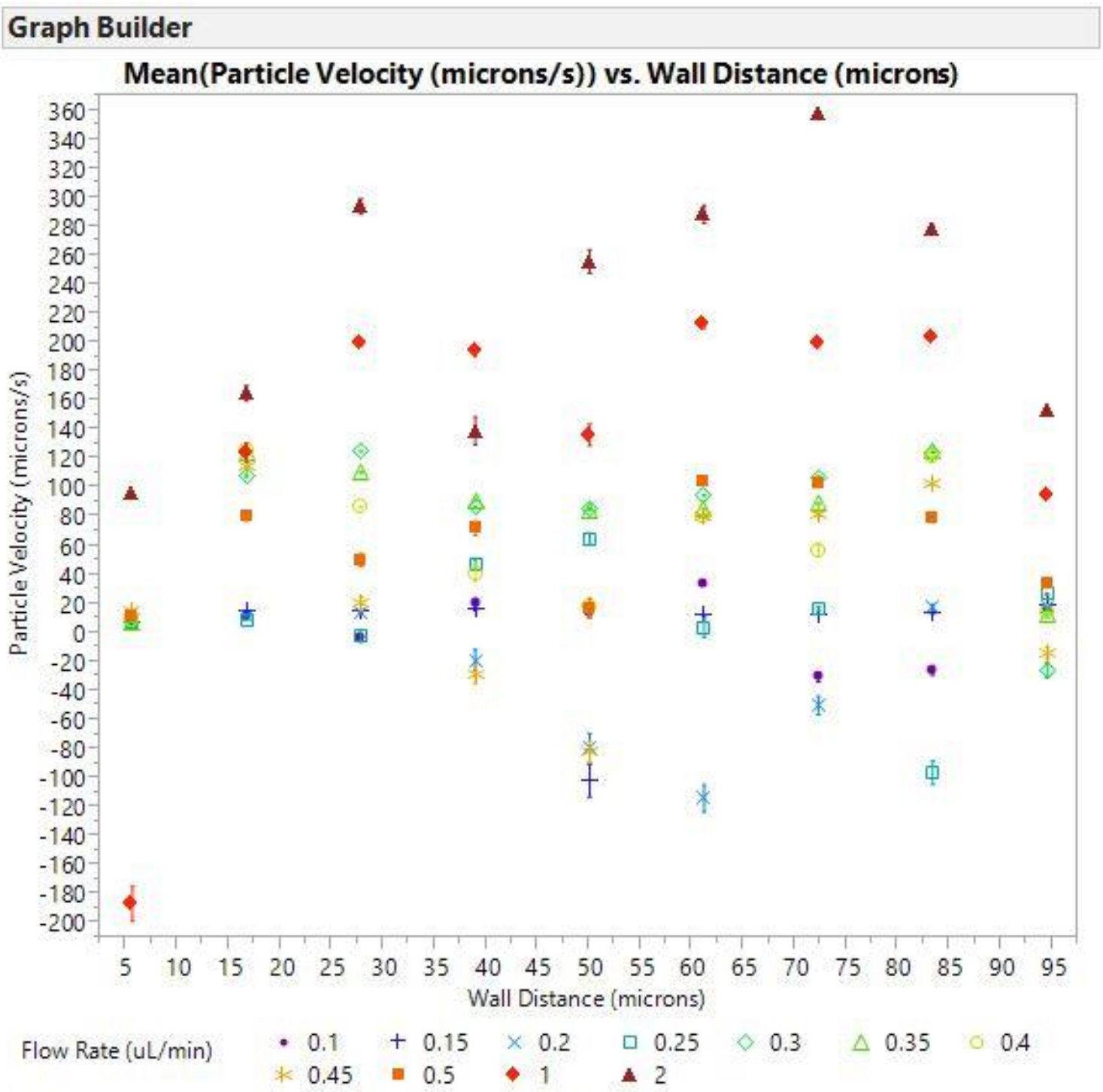

Each error bar is constructed using 1 standard error from the mean.

Figure 54. Graph for LabSmith Topas channel with New Era syringe pump. 


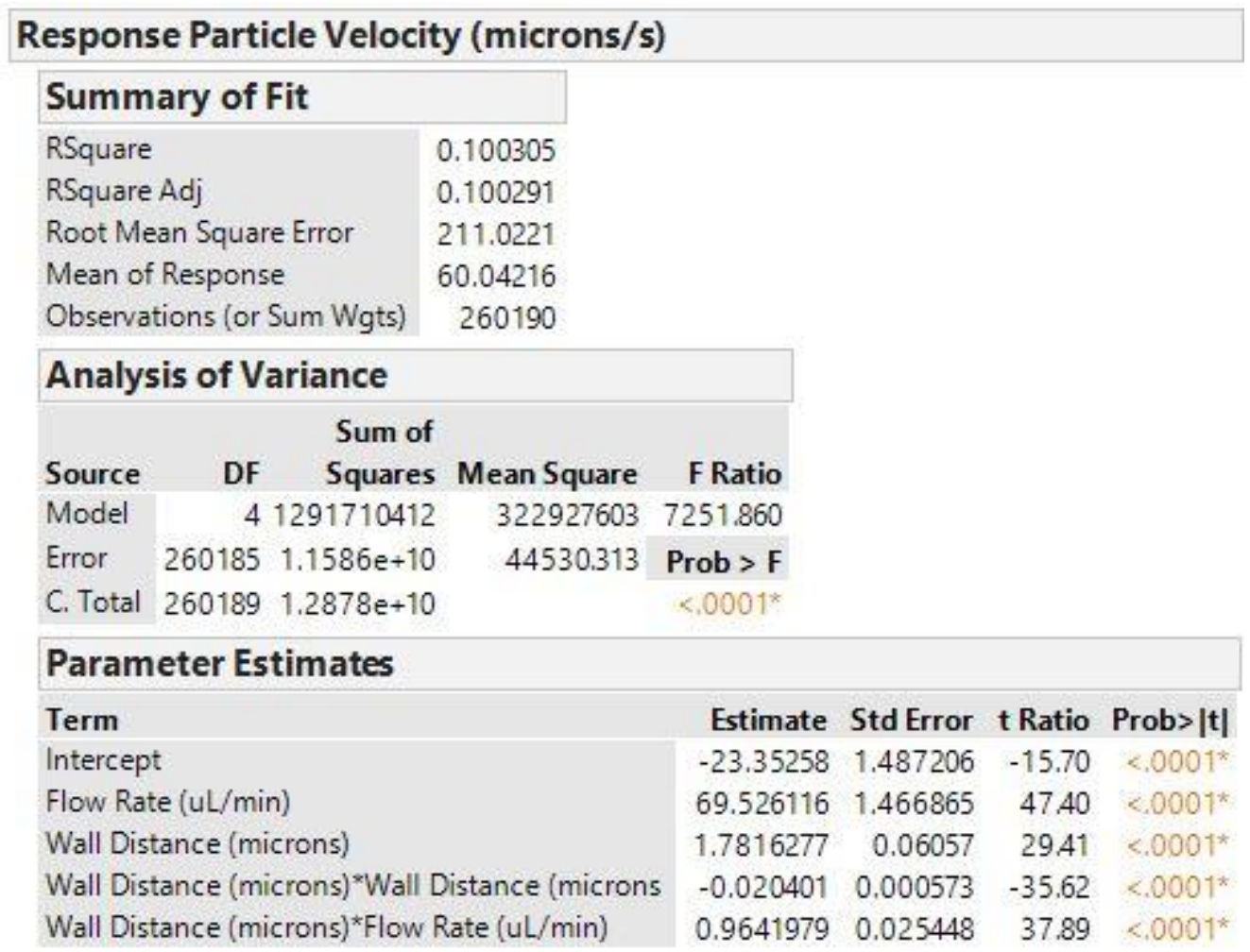

Figure 55. JMP output for model for LabSmith Topas channel with New Era syringe pump.

The LabSmith Topas channel was measured at multiple flow rates ranging from $0.5 \mu \mathrm{L} / \mathrm{min}$ to $2.5 \mu \mathrm{L} / \mathrm{min}$ with a probe size of 64 pixels by 256 pixels $(21.3 \mu \mathrm{m}$ by 82.2 $\mu \mathrm{m}$ ) using $2 \mu \mathrm{m}$ diameter fluorescent polystyrene spheres. A 20x magnification objective was used for the LabSmith Syncronized Video Microscope (Figure 56). 


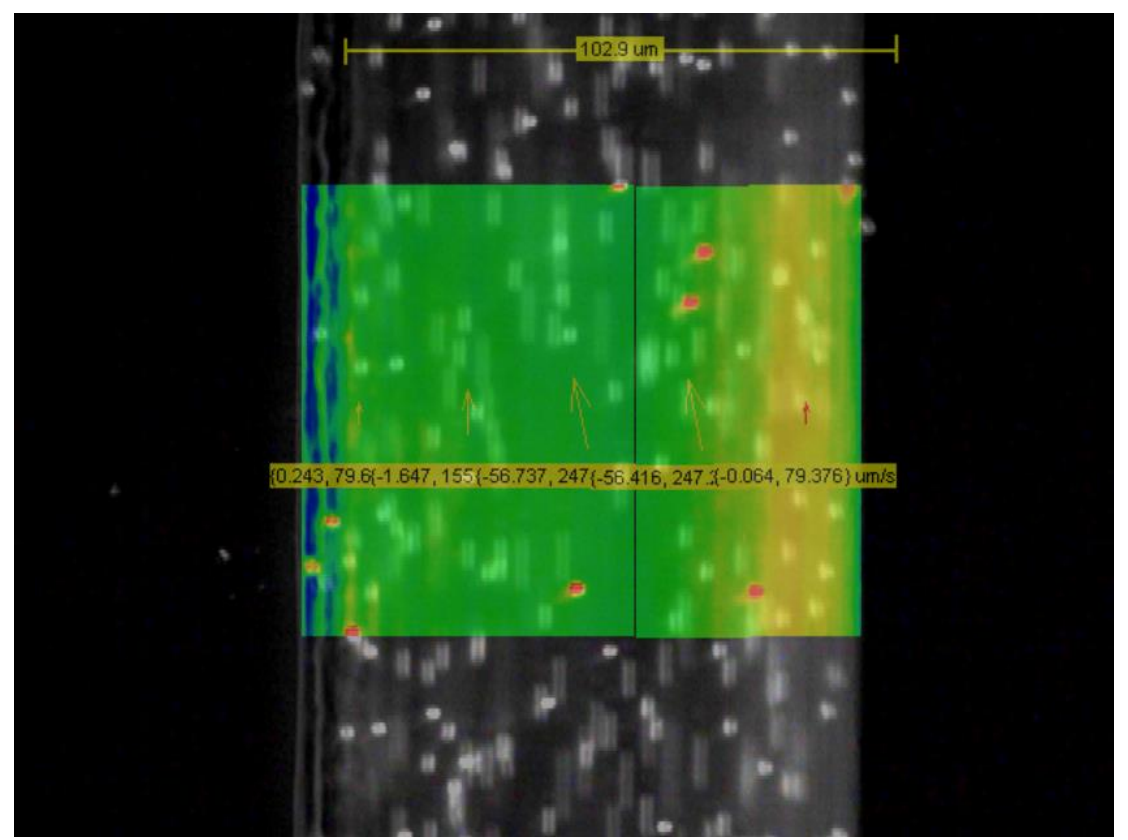

Figure 56. Probe setup within LabSmith Topas channel. Setup of probes within LabSmith Topas channel for Harvard Apparatus Syringe Pump. The probes were evenly spaced across the channel with the best size option to measure the particle velocities.

There was still some significant pulsing at $0.5 \mu \mathrm{L} / \mathrm{min}$ but this was kept in the data set to have an extra flow rate to compare. In addition, the middle flow velocity for $2.5 \mu \mathrm{L} /$ min appears to have reached the maximum flow rate measurable by the probe. Analysis was run using JMP to ensure that the visual trends seen were statistically significant (Figure 57, Figure 58). The overall ANOVA table shows a p-value less than 0.0001 indicating that the model is a good predictor of the velocities observed. In addition, the p-values of each parameter are less than 0.0001 indicating that each variable is statistically significant in predicting the flow velocity after controlling for all other variables in the model, as expected. 


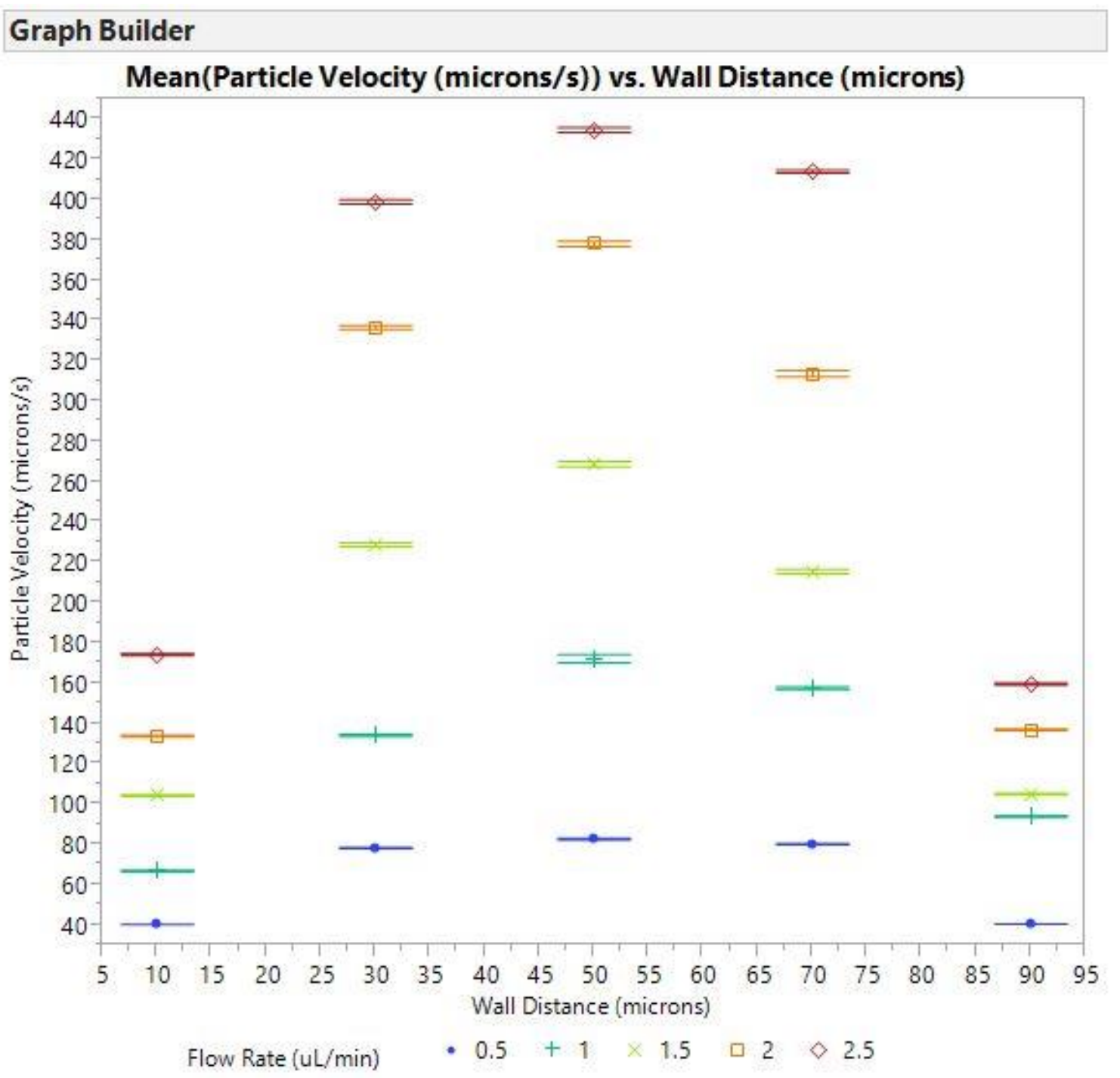

Each error bar is constructed using 1 standard error from the mean.

Figure 57. Graph for LabSmith Topas channel with Harvard Apparatus syringe pump. 


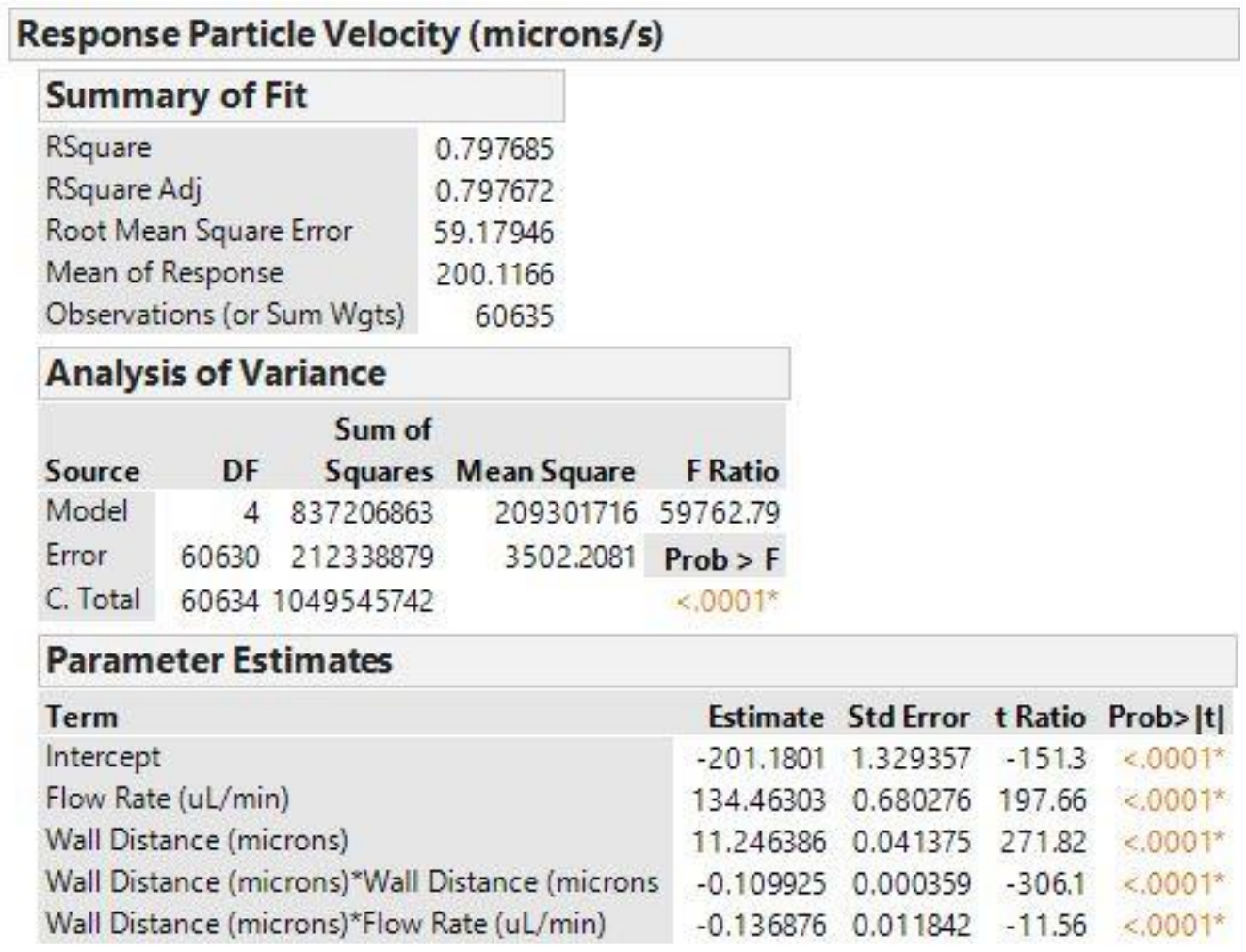

Figure 58. JMP output for model for LabSmith Topas channel with Harvard Apparatus syringe pump.

\section{LabSmith PMMA Channel}

The LabSmith PMMA channel was measured at multiple flow rates ranging from $0.1 \mu \mathrm{L} / \mathrm{min}$ to $2 \mu \mathrm{L} / \mathrm{min}$ with a probe size of 64 pixels by 256 pixels $(21.3 \mu \mathrm{m}$ by $82.2 \mu \mathrm{m})$ using $2 \mu \mathrm{m}$ diameter fluorescent polystyrene spheres. A 20x magnification objective was used for the LabSmith Syncronized Video Microscope (Figure 59). 


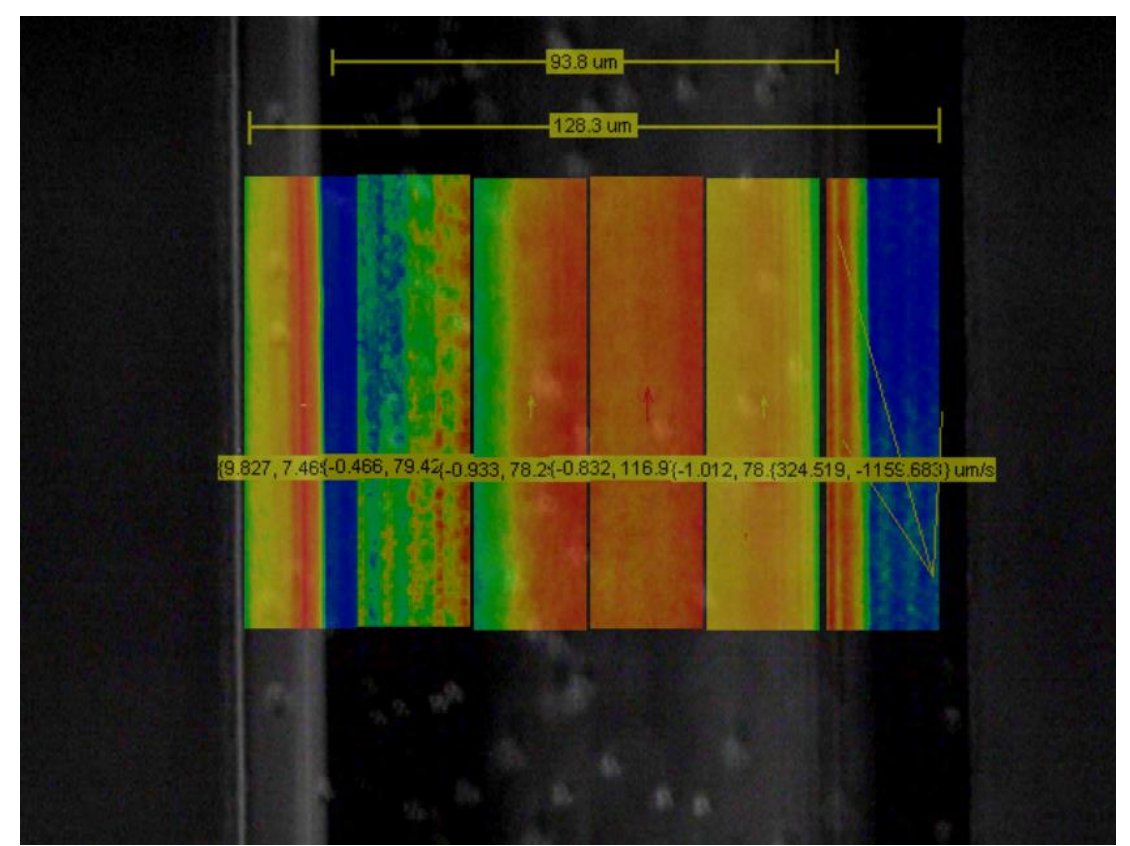

Figure 59. Probe setup within LabSmith PMMA channel. Setup of probes within LabSmith PMMA channel. The probes were evenly spaced across the channel with the best size option to measure the particle velocities.

No pattern was seen in the data although the analysis showed the flow rates and position within the channel were significant (Figure 60, Figure 61). 


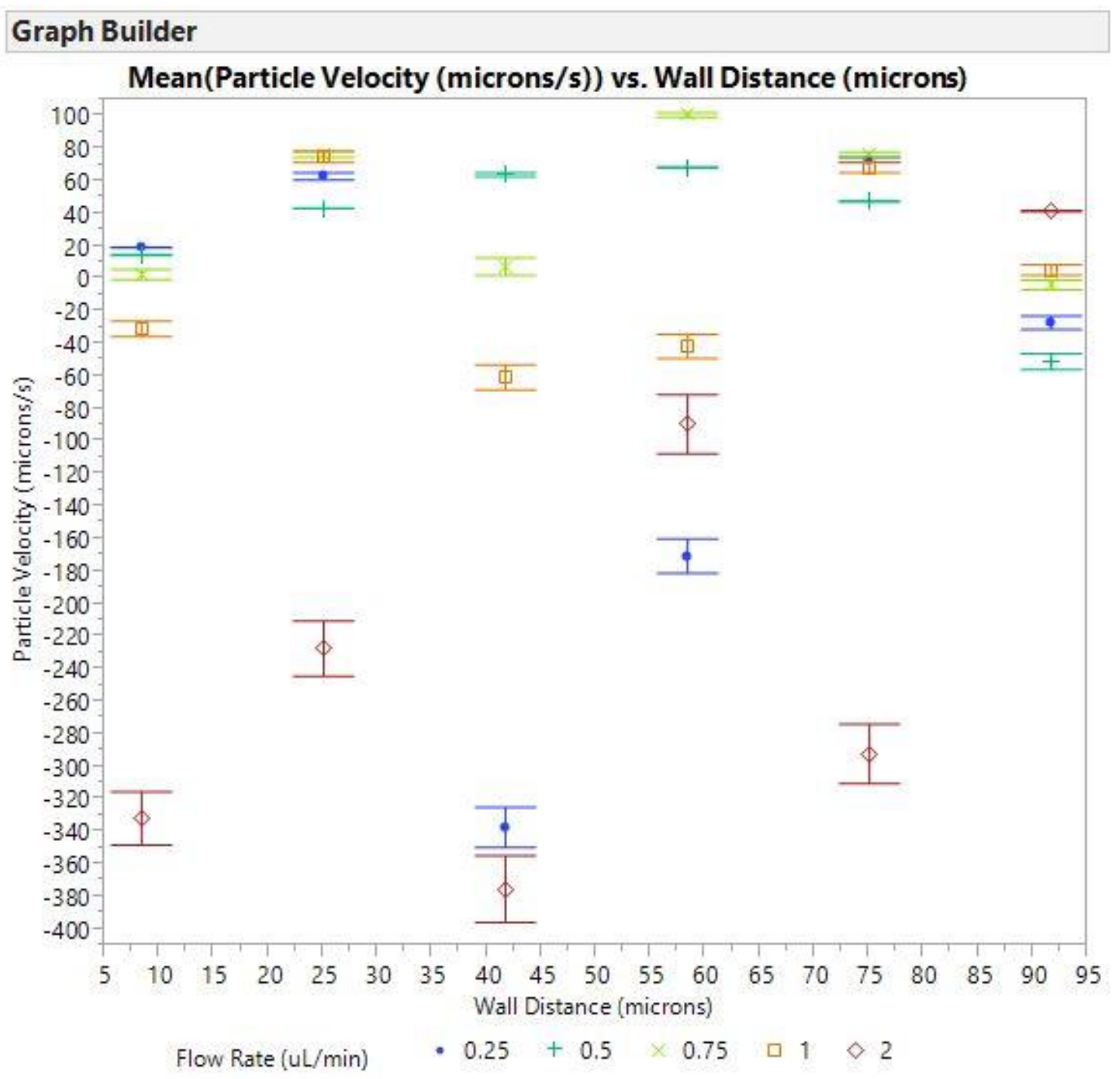

Each error bar is constructed using 1 standard error from the mean.

Figure 60. Graph of LabSmith PMMA channel with New Era syringe pump. 


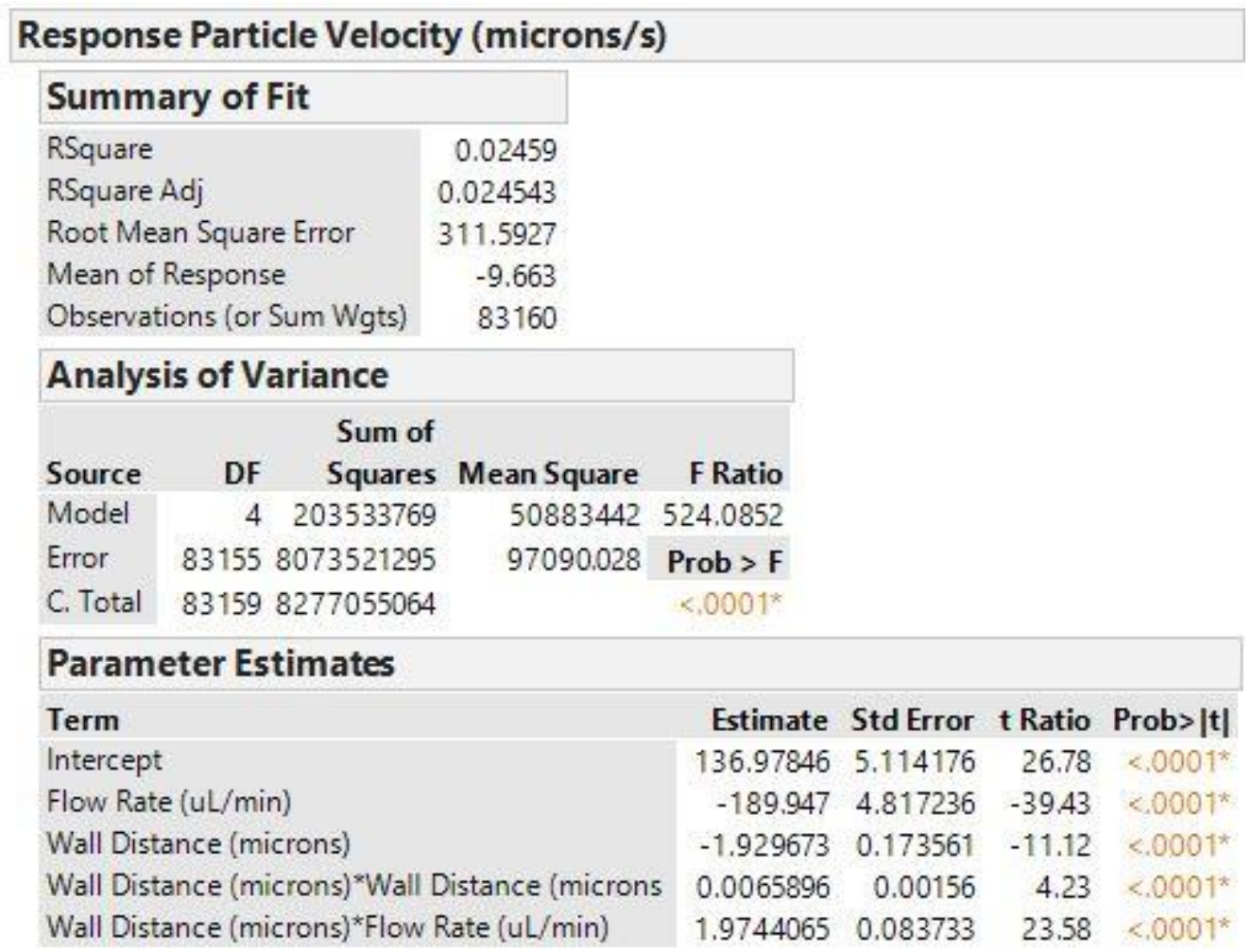

Figure 61. JMP output for model for LabSmith PMMA channel with New Era syringe pump.

Since pulsing was significantly affecting the measurements, the Harvard Apparatus Syringe Pump was used to try to reduce this issue. The LabSmith PMMA channel was measured at multiple flow rates ranging from $0.5 \mu \mathrm{L} / \mathrm{min}$ to $2.5 \mu \mathrm{L} / \mathrm{min}$ with the same channel and microscope setup as for the New Era Syringe Pump measurements. This data showed a somewhat parabolic shape but there is still some overlap between the different flow rates (Figure 62, Figure 63). The channel was slightly warped which prevented it from sitting on a flat surface well. This could have interfered with finding the middle plane of the channel which would give measurements with less wall interference. 


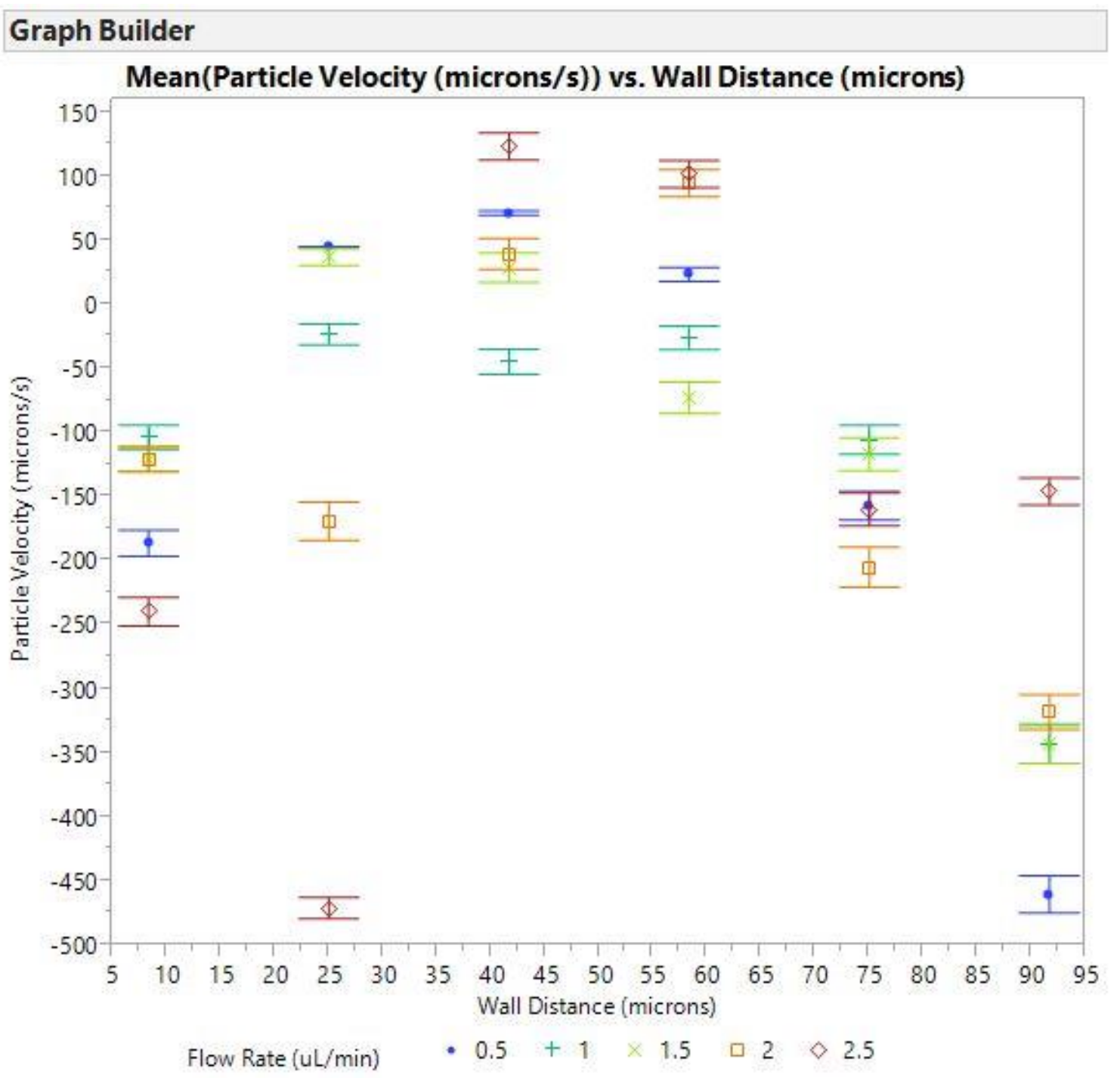

Each error bar is constructed using 1 standard error from the mean.

Figure 62. Graph of LabSmith PMMA channel with Harvard Apparatus syringe pump. 


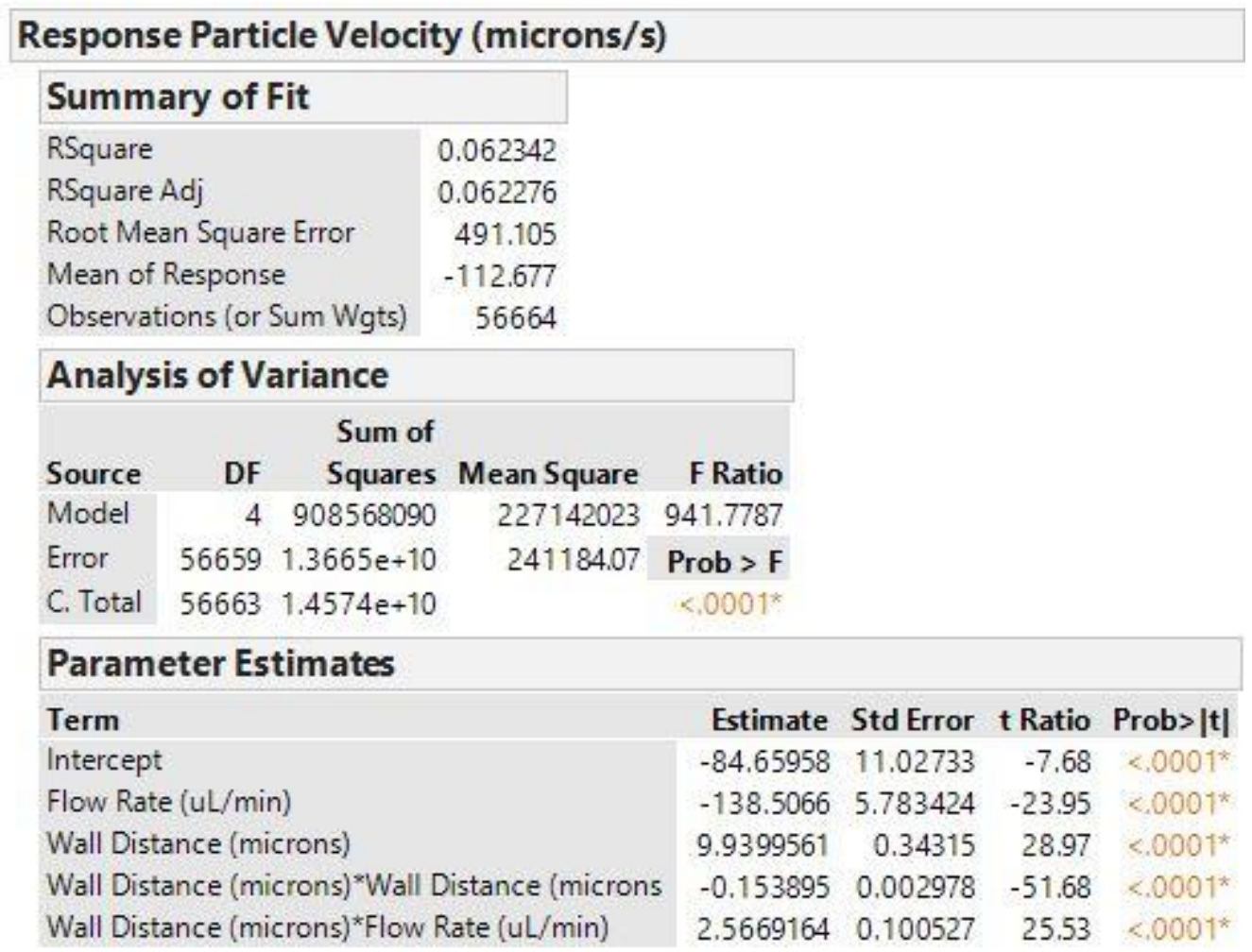

Figure 63. JMP output for model for LabSmith PMMA channel with Harvard Apparatus syringe pump.

\subsubsection{Discussion}

\section{Translume Channel}

The average overall velocity in the channel was compared for each flow rate (Table III). The mean and median velocities are somewhat similar for each syringe pump type. Slight differences could be from different calibrations for each syringe pump or a different plane of the channel being measured. In general, the mean velocity is smaller than the median. This is probably because some particles were always measured much slower than the majority of the particles. This is either because of a reaction with the wall or the particle going too fast for the equipment to pick up. These lagging measurements would have skewed the mean more than the median by definition. Finally, the $5 \mu \mathrm{L} / \mathrm{min}$ flow rate 
decreases for the New Era Syringe Pump. Recall that the graph of the velocities showed that the center channel velocities dropped significantly. These velocities were believed to be on the boundary of the equipment's measurement capabilities which caused only the slower particles to be detected. In addition, a theoretical average velocity was calculated by dividing the flow rate by the channel's cross-sectional area. In general, the measured velocities were higher than the theoretical velocity, possibly from equipment mis-calibration or the effect of the parabolic flow profile on the particle velocities.

Table III. Translume pressure-driven results summary. The mean and median velocity for each type of syringe pump was calculated for each flow rate to compare with the theoretical velocity.

\begin{tabular}{cccccc}
\hline $\begin{array}{c}\text { Flow Rate } \\
(\mu \mathrm{L} / \mathrm{min})\end{array}$ & Theoretical & \multicolumn{4}{c}{ Average Velocity $(\mu \mathrm{m} / \mathbf{s})$} \\
& & Mean & Median & \multicolumn{2}{c}{ Harvard Apparatus } \\
Mean & Median \\
\hline $\mathbf{1}$ & 185.1851852 & 251.79 & 307.209 & 243.43 & 231.08 \\
$\mathbf{1 . 5}$ & 277.7777778 & 390.83 & 459.782 & 360.32 & 383.69 \\
$\mathbf{2}$ & 370.3703704 & 508.27 & 612.006 & 586.55 & 613.36 \\
$\mathbf{2 . 5}$ & 462.962963 & 663.53 & $765.64 \mathrm{f}$ & 619.11 & 689.22 \\
$\mathbf{3}$ & 555.5555556 & 809.85 & 938.5 & 754.89 & 1015.05 \\
$\mathbf{3 . 5}$ & 648.1481481 & 943.81 & 1091.95 & 927.07 & 1168.07 \\
$\mathbf{4}$ & 740.7407407 & 1050.66 & 1168.76 & 1000.16 & 1168.57 \\
$\mathbf{4 . 5}$ & 833.3333333 & 1133.68 & 1322.22 & $\mathrm{~N} / \mathrm{A}$ & $\mathrm{N} / \mathrm{A}$ \\
$\mathbf{5}$ & 925.9259259 & 927.61 & 613.55 & 1235.74 & 1648.28 \\
\hline
\end{tabular}

The parabolic profile was modeled using Poiseuille-flow which takes into account some surface interactions (Equation 16) [44].

$$
v_{x}(y, z)=\frac{4 h^{2} \Delta p}{\pi^{3} \eta L} \sum_{n, o d d}^{\infty} \frac{1}{n^{3}}\left[1-\frac{\cosh \left(\frac{n \pi y}{h}\right)}{\cosh \left(\frac{n \pi w}{2 h}\right)}\right] \sin \left(\frac{n \pi z}{h}\right)
$$

Where $h$ is the channel's height ( $300 \mu \mathrm{m}$ for Translume channel), $w$ is the

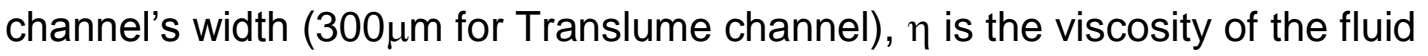
$\left(0.001 \mathrm{~kg} / \mathrm{m}^{*} \mathrm{~s}\right), L$ is the channel's length (38mm for Translume channel), $n$ is a 
series of odd numbers, $y$ is the position along the width of the channel measured from the center, $z$ is the position along the height of the channel measured from the wall, and $\Delta p$ is calculated by:

Equation 17. Determination of pressure.

$$
\Delta p=\frac{12 Q \eta L}{h^{3} w\left[1-\sum_{n, o d d}^{\infty} \frac{1}{n^{5}} \frac{192}{\pi^{5}} \frac{h}{w} \tanh \left(\frac{n \pi w}{2 h}\right)\right]}
$$

Where $Q$ is the volumetric flow rate.

This model was performed at varying points within the Translume channel at a $2.5 \mu \mathrm{L} / \mathrm{min}$ flow rate using 10 values of $n$ (Figure 64).

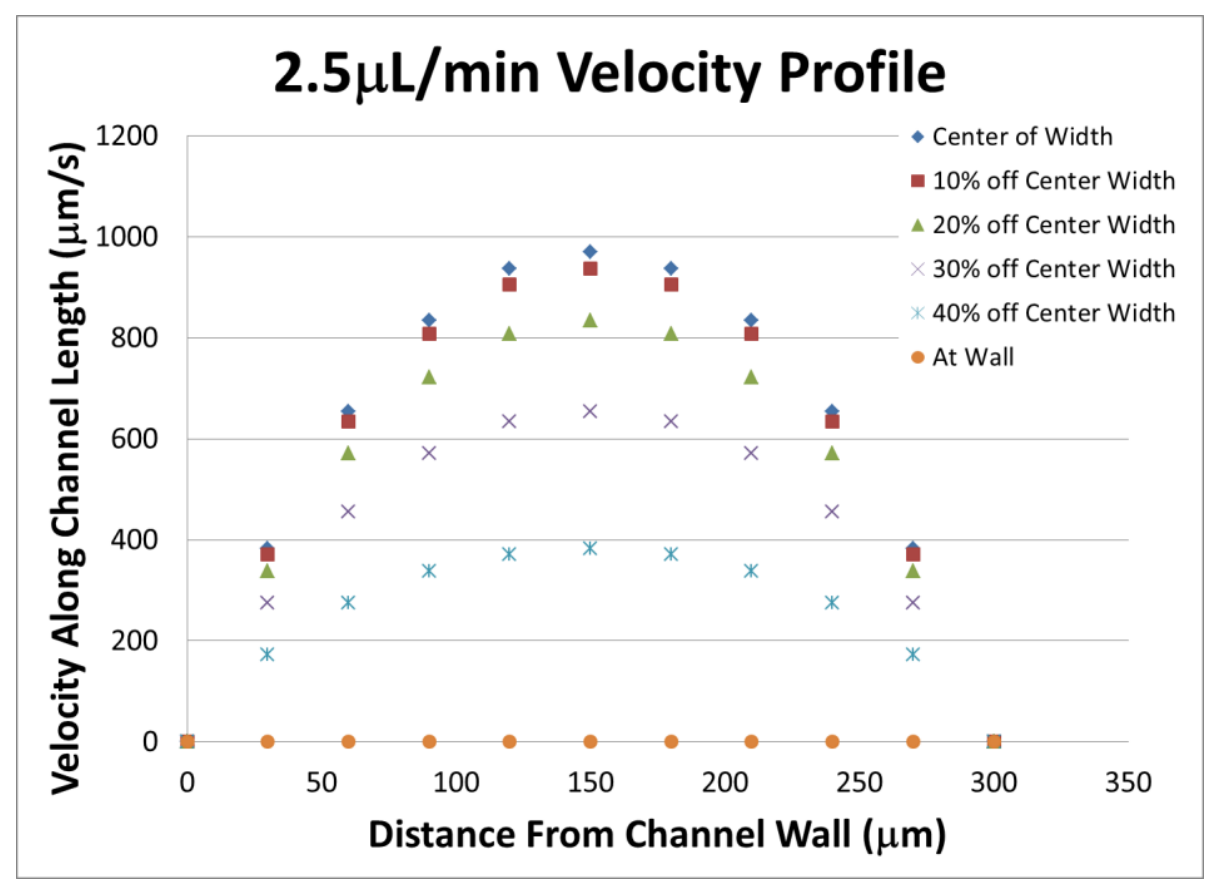

Figure 64. Model of parabolic profile in Translume channel.

This data was then normalized by dividing the velocity at a point by the max velocity for its plane and by dividing the wall distance by the channel width 
(Figure 65). In this graph all the curves overlap to form one curve. Slight misalignments are probably from the limited number of $n$ values used.

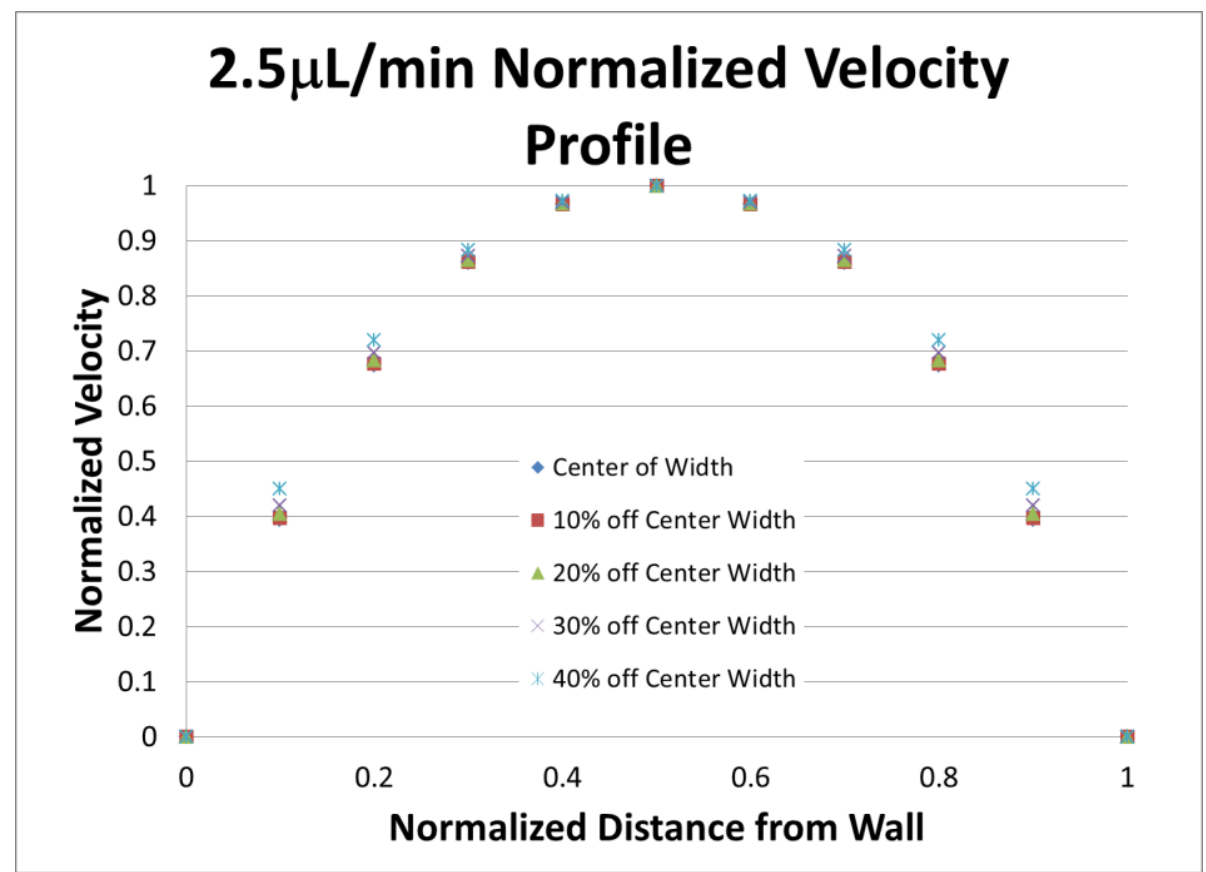

Figure 65. Normalized model of parabolic profile.

The center plane theoretical data was plotted with the collected data for the $2.5 \mu \mathrm{L} / \mathrm{min}$ (Figure 66). The measured data appears to fit the model very well. There is a slight misalignment to the data on the right which could be from uneven spacing of the probes or from the microscope stage being tilted and viewing multiple planes while measuring. 


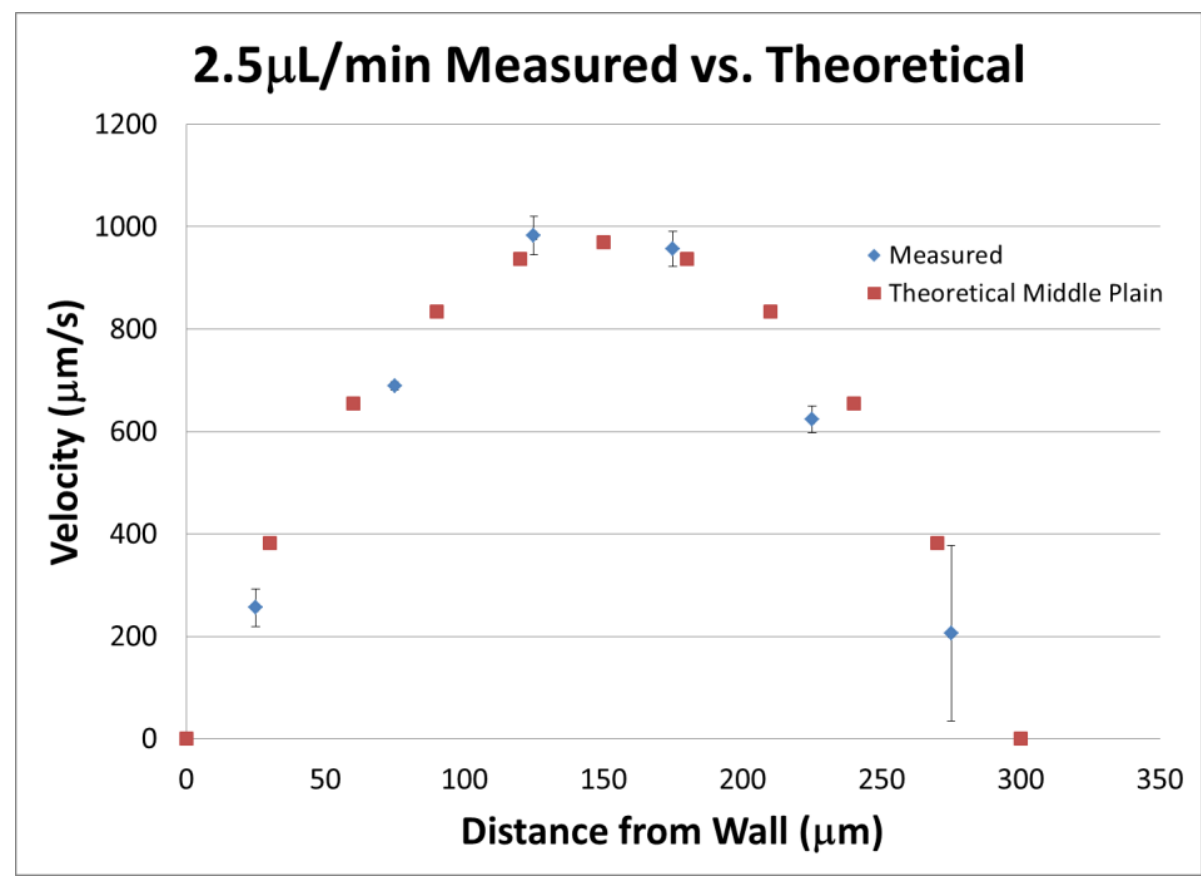

Figure 66. Measured vs. theoretical $2.5 \mu \mathrm{L} / \mathrm{min}$ Translume data. The error bars are constructed using 1 standard deviation from the mean.

\section{Cidra Channel}

The average overall velocity in the channel was compared for each flow rate (Table IV). As with the Translume channel, the means and medians are comparable with the means slightly lower than the medians. In addition, there is a decrease in velocity for the $15 \mu \mathrm{L} / \mathrm{min}$ flow rate with the Harvard Apparatus Syringe Pump probably from fast particles not being detected accurately. This data shows a huge difference between the two different syringe pumps.

Originally it was believed that this was a calibration issue, but channel widths measured at the time of the velocity measurements showed similar results. This could be from different planes within the channel or from a different particle solution being used to take the measurements. There is a microscope setting that allows for different size particles to be used with similar results that was used, but this still showed great differences. It appears that the smaller bead 
sizes resulted in observed velocities closer to the theoretical velocity for the given flow rates. Bubbles also stuck to side walls of the channel very easily, even with the wetting solution. The channel was tilted while originally filling with particles to help fill completely. The bubbles might have an effect on the velocities since it changes the dimensions that the fluid is running through.

Table IV. Cidra pressure-driven results summary. The mean and median velocity for each type of syringe pump was calculated for each flow rate to compare with the theoretical velocity.

\begin{tabular}{|c|c|c|c|c|c|}
\hline \multirow{3}{*}{$\begin{array}{c}\text { Flow Rate } \\
(\mu \mathrm{L} / \mathrm{min})\end{array}$} & \multicolumn{5}{|c|}{ Average Velocity $(\mu \mathrm{m} / \mathrm{s})$} \\
\hline & \multirow[t]{2}{*}{ Theoretical } & \multicolumn{2}{|c|}{ New Era } & \multicolumn{2}{|c|}{ Harvard Apparatus } \\
\hline & & Mean & Median & Mean & Median \\
\hline 2.5 & 166.6666667 & 6.58 & 6.57 & 61.6 & 191.91 \\
\hline 5 & 333.3333333 & 7.07 & 6.93 & 220.08 & 345.13 \\
\hline 7.5 & 500 & 26.08 & 38.4 & 514.43 & 583.89 \\
\hline 10 & 666.6666667 & 32.87 & 39 & 692.79 & 775.82 \\
\hline 12.5 & 833.3333333 & 32.99 & 39.43 & 701.25 & 824.06 \\
\hline 15 & 1000 & 36.16 & 39.8 & 509.19 & 566.02 \\
\hline
\end{tabular}

\section{LabSmith Channels}

The average overall velocities in the channels were compared for each flow rate (Table V, Table VI). These channels have the same dimensions and should theoretically have the same average flow velocities; however, very different average velocities were measured for each channel and these velocities are very different from the theoretical velocity. First, the Topas channel has fairly consistent measurements between the New Era and Harvard Apparatus syringe pumps with most means lower than the median. However, the measured velocities are much lower than the theoretical velocities. It is believed that the interaction with the wall might be strong enough to slow down the particles even in the middle plane of a channel that is this small or that only the lagging particles were getting measured because the majority of the particles were going way too fast to be detected. Second, the PMMA channel does not show any consistent 
trend in the data and even has negative mean velocities. This indicated that the particles were going too fast to be detected. In addition, this channel was badly warped which might have interfered with finding the middle plane. Overall, it appears that channel of this dimension $(100 \mu \mathrm{m}$ by $100 \mu \mathrm{m})$ is too small to get accurate measurements.

Table V. LabSmith Topas pressure-driven results summary. The mean and median velocity for each type of syringe pump was calculated for each flow rate to compare with the theoretical velocity.

\begin{tabular}{|c|c|c|c|c|c|}
\hline \multirow{3}{*}{$\begin{array}{c}\text { Flow } \\
\text { Rate } \\
(\mu \mathrm{L} / \mathrm{min})\end{array}$} & \multirow{3}{*}{$\begin{array}{c}\text { Theoretical } \\
(\mu \mathrm{m} / \mathrm{s})\end{array}$} & \multicolumn{4}{|c|}{ Topas Average Velocity $(\mu \mathrm{m} / \mathrm{s})$} \\
\hline & & $\mathrm{Ne}$ & Era & Harvars & Apparatus \\
\hline & & Mean & Median & Mean & Median \\
\hline 0.5 & 33 & 61.55 & 81.51 & 64.54 & 78.43 \\
\hline 1 & 166 & 131.37 & 204.25 & 125.18 & 117.08 \\
\hline 1.5 & 2500 & $\mathrm{~N} / \mathrm{A}$ & $\mathrm{N} / \mathrm{A}$ & 184.72 & 192.99 \\
\hline 2 & 3333.333 & 225.53 & 347.51 & 260.08 & 285.58 \\
\hline 2.5 & 4166.667 & $\mathrm{~N} / \mathrm{A}$ & $N / A$ & 316.59 & 400.48 \\
\hline
\end{tabular}

Table VI. LabSmith PMMA pressure-driven results summary. The mean and median velocity for each type of syringe pump was calculated for each flow rate to compare with the theoretical velocity.

\begin{tabular}{|c|c|c|c|c|c|}
\hline \multirow{3}{*}{$\begin{array}{c}\text { Flow } \\
\text { Rate } \\
(\mu \mathrm{L} / \mathrm{min})\end{array}$} & \multirow{3}{*}{$\begin{array}{c}\text { Theoretical } \\
(\mu \mathrm{m} / \mathrm{s})\end{array}$} & \multicolumn{4}{|c|}{ PMMA Average Velocity $(\mu \mathrm{m} / \mathrm{s})$} \\
\hline & & \multicolumn{2}{|c|}{ New Era } & \multicolumn{2}{|c|}{ Harvard Apparatus } \\
\hline & & Mean & Median & Mean & Median \\
\hline 0.5 & 833.3333 & 31.52 & 40.9 & -110.37 & 41.07 \\
\hline 1 & 1666.667 & 2.86 & 78.2 & -107.4 & 77.99 \\
\hline 1.5 & 2500 & $\mathrm{~N} / \mathrm{A}$ & $\mathrm{N} / \mathrm{A}$ & -97.82 & 78.55 \\
\hline 2 & 3333.333 & -212.16 & 41.092 & -112.87 & 79.15 \\
\hline 2.5 & 4166.667 & $\mathrm{~N} / \mathrm{A}$ & $\mathrm{N} / \mathrm{A}$ & -131.64 & 70.45 \\
\hline
\end{tabular}

\subsection{ELECTRO-KINETIC DRIVEN FLOW}

The Translume channel had the best pressure-driven velocity profile, so this channel was used to obtain electro-kinetic flow for comparison.

\subsubsection{Results and Analysis}

The Translume channel was measured at multiple voltages ranging from $50 \mathrm{~V}$ to $250 \mathrm{~V}$ with a probe size of 64 pixels by 256 pixels $(42.7 \mu \mathrm{m}$ by $163.7 \mu \mathrm{m})$ using 
$1 \mu \mathrm{m}$ diameter fluorescent polystyrene spheres. A 10x magnification objective was used for the LabSmith Syncronized Video Microscope (Figure 67). Above $250 \mathrm{~V}$, the particles started sticking to the walls of the channel in large clumps making it difficult to view movement so this was used as the upper limit for testing.

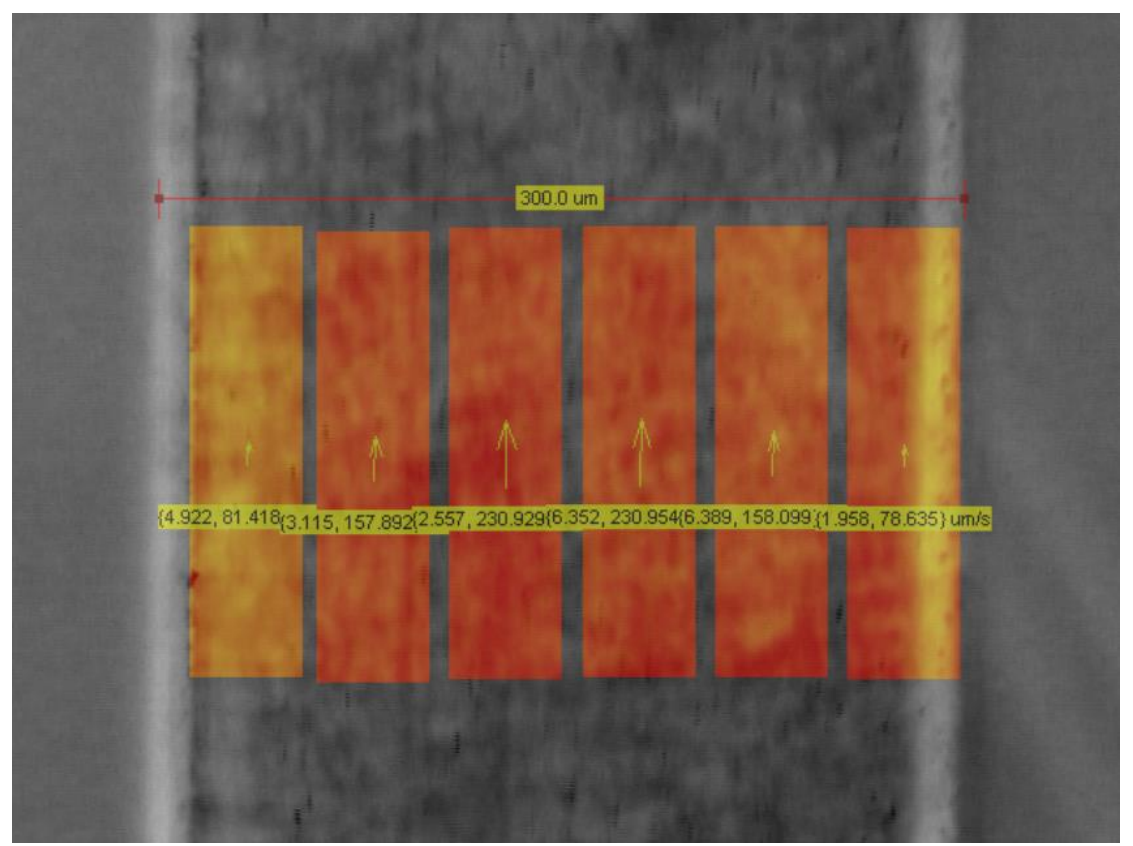

Figure 67. Probe setup within Translume channel. Setup of probes within Translume channel. The probes were evenly spaced across the channel with the best size option to measure the particle velocities.

The collected data shows a parabolic profile; although, this profile seems less extreme than for the pressure-driven flow (Figure 68, Figure 69). There also appears to be an equal spacing between the voltages except for the $225 \mathrm{~V}$ and $250 \mathrm{~V}$. At the higher voltages, the particles velocities would increase to a maximum and then flip directions after a fairly short period of time probably from pressure build up. For this reason, only small data files could be obtained for the higher voltages. It is possible that even with this smaller file size, some of the flipped data still got into the set. It is not believed that this significantly affected 
the data but it is possible that the particle velocities and fluid flow was not able to reach its full speed before the flow direction flipped, which could account for why the higher voltages show similar velocities. Even at the lower velocities, there would be some backward movement near the walls. According to the JMP analysis, both the distance from the wall and the voltage have significant effects on the observed velocity.

\section{Graph Builder}

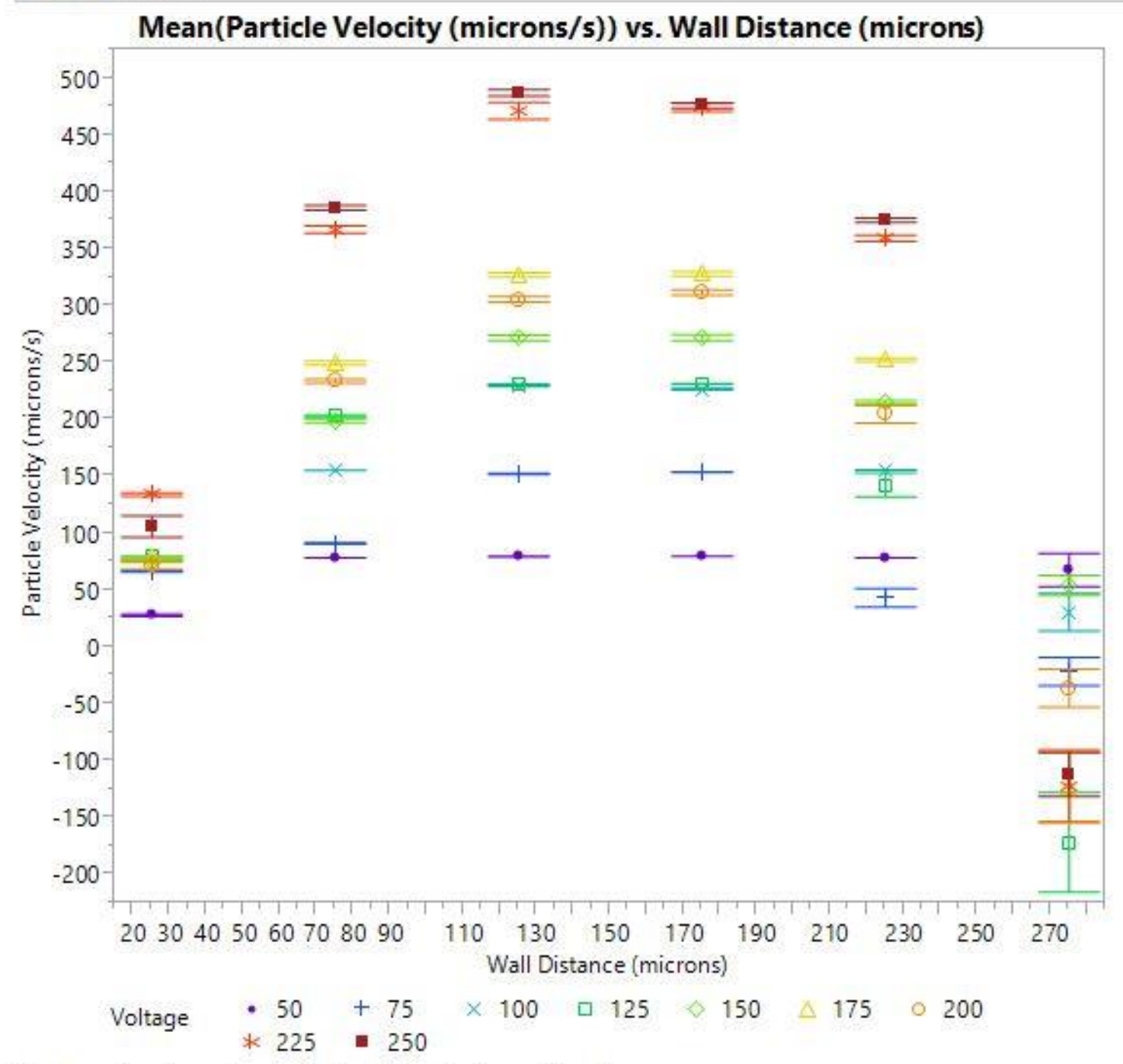

Each error bar is constructed using 1 standard error from the mean.

Figure 68. Graph of Translume channel with electro-kinetic flow. 


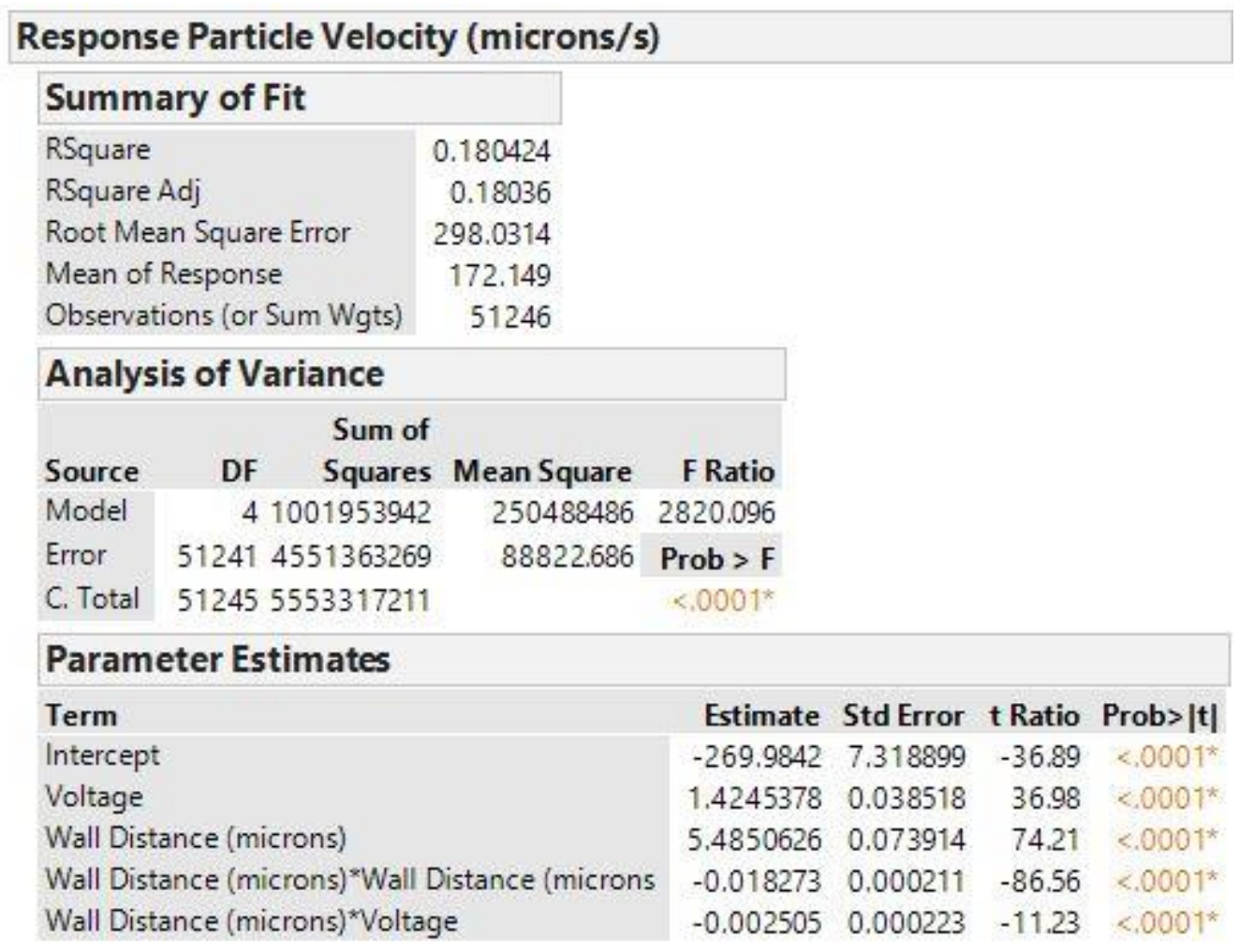

Figure 69. JMP output for model for Translume channel with electro-kinetic flow.

\subsubsection{Discussion}

The expected electro-osmotic velocity could be calculated by the Helmholtz-

Smoluchowski equation with a zeta potential of $100 \mathrm{mV}$ for the glass surface and $34.2 \mathrm{mV}$ for the PS beads in a 7.00pH solution (Equation 13) [45] [46]. The Debye length was considered small compared to the particle size. The total theoretical velocity was calculated by summing the electro-osmotic and electrophoretic effects of a voltage on the system. The average overall velocity in the channel was compared for each applied voltage (Table VII). The means and medians are fairly equivalent over the different applied voltages; however, the means are consistently slightly lower than the medians. The backwards moving particles at the wall might have been moving faster at the higher voltages which would decrease the overall average more than it would affect the median. In addition, 
$150 \mathrm{~V}, 175 \mathrm{~V}$, and $200 \mathrm{~V}$ all have similar average velocities even though the graph clearly showed separate velocity profiles across the channel. This could be from the backward moving wall particles or from a quicker buildup of pressure slowing down some particles.

Table VII. Translume electro-kinetic driven results summary. The mean and median velocity for each flow rate.

\begin{tabular}{cccccc}
\hline Voltage Applied (V) & $\begin{array}{c}\text { Average Velocity }(\mu \mathrm{m} / \mathbf{s}) \\
\text { Theoretical } \\
\text { EO }\end{array}$ & $\begin{array}{c}\text { Theoretical } \\
\text { EP }\end{array}$ & $\begin{array}{c}\text { Theoretical } \\
\text { Total }\end{array}$ & Mean & Median \\
\hline $\mathbf{5 0}$ & 88.54 & -30.28068 & 58.25932 & 69.17 & 79.01 \\
$\mathbf{7 5}$ & 132.81 & -45.42102 & 87.38898 & 81.37 & 81.32 \\
$\mathbf{1 0 0}$ & 177.08 & -60.56136 & 116.51864 & 146.48 & 156.10 \\
$\mathbf{1 2 5}$ & 221.35 & -75.7017 & 145.6483 & 119.89 & 158.49 \\
$\mathbf{1 5 0}$ & 265.62 & -90.84204 & 174.77796 & 181.77 & 230.54 \\
$\mathbf{1 7 5}$ & 309.89 & -105.98238 & 203.90762 & 186.12 & 232.05 \\
$\mathbf{2 0 0}$ & 354.16 & -121.12272 & 233.03728 & 182.47 & 231.18 \\
$\mathbf{2 2 5}$ & 398.43 & -136.26306 & 262.16694 & 281.20 & 383.10 \\
$\mathbf{2 5 0}$ & 442.7 & -151.4034 & 291.2966 & 286.93 & 383.00 \\
\hline
\end{tabular}

The measured values are close to the theoretical velocities calculated (Figure 70). Slight differences could be from differences in zeta potential in this system from similar ones reported in literature. Again, there is some variation in the measured data but this is believed to be from the pressure buildup as the fluid would flow. 


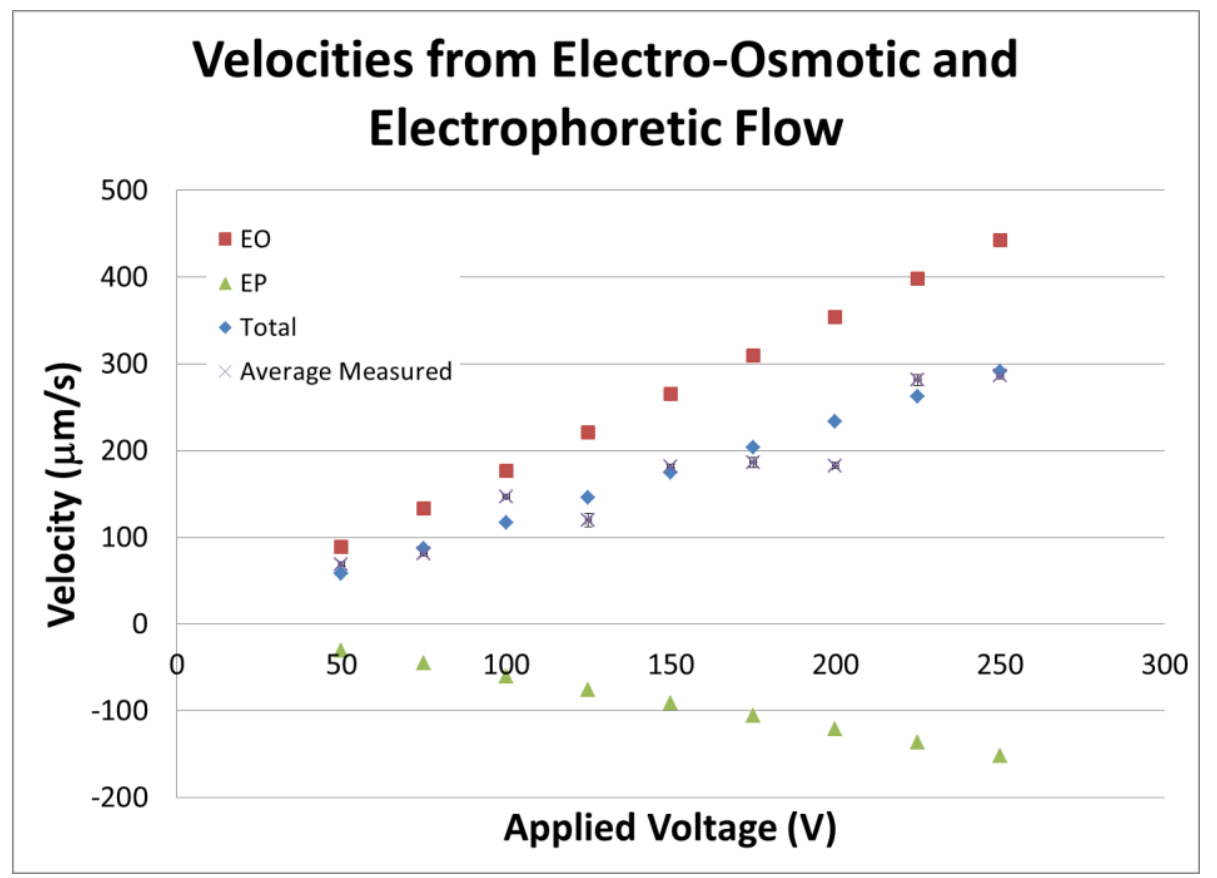

Figure 70. Plot of effects of electro-osmotic and electrophoretic flow. Error bars are constructed using 1 standard error from the mean.

\subsection{FLOW COMPARISONS}

The pressure-driven and electro-kinetic driven velocity profiles were compared. A mid-range flow rate and voltage was selected and graphed together using JMP (Figure 71). Before both flow methods showed parabolic profiles; however, the parabola for the electro-kinetic flow is much less curved than the pressure-driven flow. It is easy to see why pressure-driven flows are said to have parabolic flow profiles while electro-kinetic flows have plug shaped profiles. 


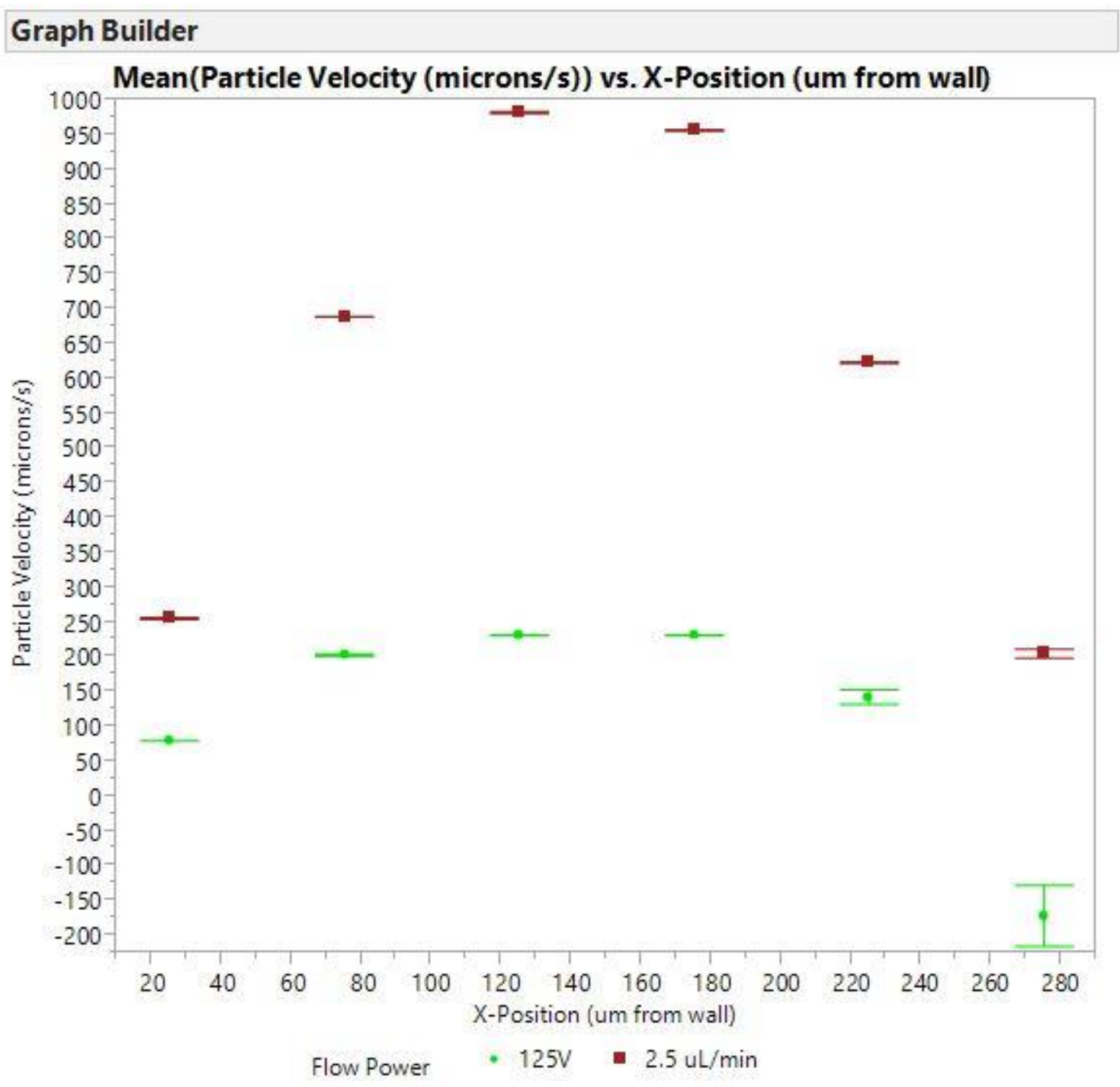

Each error bar is constructed using 1 standard error from the mean.

Figure 71. Graph of Translume channel velocity profile for pressure-driven and electro-kinetic driven flow. The $125 \mathrm{~V}$ electro-kinetic flow at about $200 \mu \mathrm{m} / \mathrm{s}$ is comparable with a $1 \mu \mathrm{L} / \mathrm{min}$ flow rate. 


\section{Chapter 6 Summary and Conclusion}

\subsection{FINDINGS}

For the pressure-driven flow, the Harvard Apparatus (Catalog\#: 70-2211) syringe pump was found to pulse much less at low flow rates than the New Era (Model\#: NE-300) syringe pump. This was greatly influential on the lower flow rates where pulsing caused measurement issues. The two syringe pumps had similar calibrations but measured velocities were greatly influenced by wall interactions so variations in the plane within the channel caused variations in measured velocities. In addition, particle size might influence wall interactions and have an effect on the measured velocities. The measured data also agreed with the Poiseuille-flow model for a rectangular chip for the Translume channel.

As expected, channel size and dimensions had an influence on velocity profile. The Translume channel with dimensions of $300 \mu \mathrm{m}$ by $300 \mu \mathrm{m}$ showed the best parabolic profile while the LabSmith channels had more difficulty with showing the parabolic profile with a $100 \mu \mathrm{m}$ by $100 \mu \mathrm{m}$ square cross-section. This is possibly due to wall interactions because of the smaller dimensions. Finally, the Cidra channel with dimensions $2.5 \mathrm{~mm}$ by $100 \mu \mathrm{m}$ showed some slower velocities right next to the wall but the profile was very inconsistent. It is also possible that the $100 \mu \mathrm{m}$ thickness is too small of a depth to focus on with the microscope. The focusing setting was very coarse and it is possible that the middle of the channel was just skipped over. These pancake shaped channels that are much wider than they are thick are much easier to fabricate using soft lithography, but they do not give good profiles for comparison. 
The $1 \mu \mathrm{m}$ particles showed good results for multiple channels but are hard to focus on well. There were minor issues with clumping at higher voltages for the electro-kinetic flow but these could mostly be avoided. Larger particles may need to be purchased if using the $4 x$ magnification objective since these particles may be too small for the probes to detect. In addition, a $7.00 \mathrm{pH}$ buffer helped with reducing particles sticking to the wall, but a $4.01 \mathrm{pH}$ buffer helped clean out particles that had already stuck to the wall. The measured electro-kinetic flow also agreed with models taking into account both electro-osmosis and electrophoresis.

In the future, I would recommend continuing to use the $300 \mu \mathrm{m}$ square crosssection channel to observe flow profiles on the 10x magnification objective. I believe smaller channel dimensions would make it difficult to measure the velocity profile because of wall interactions. Large channels could be measured keeping in mind the field of view for the $10 x$ magnification objective is $418 \mu \mathrm{m}$ wide by $305 \mu \mathrm{m}$ long and the field of view for the $4 \mathrm{x}$ magnification objective is $1048 \mu \mathrm{m}$ wide by $761 \mu \mathrm{m}$ long. However, fluid flow should be kept in the length direction because using wide probes tends to freeze the camera feed of the microscope. Care should be taken with the $4 \mathrm{x}$ magnification objective and thicker channels because the DOF may not allow focusing on a center plane.

\subsection{LIMITATIONS}

There were some limitations from the equipment during this study. First, the syringe pumps pulsed at low flow rates, limiting the range that can be measured 
accurately. In addition, the microscope probes cannot measure particles moving too fast to correlate accurately. The max flow rate that can be measured depends on the objective being used. The lower magnification objectives can measure much higher velocities than the higher magnification objectives. For example, the $10 x$ objective can measure a maximum velocity of about $1700 \mu \mathrm{m} / \mathrm{s}$, while the $20 \mathrm{x}$ objective can measure a maximum velocity of about $450 \mu \mathrm{m} / \mathrm{s}$. Finally, the power supply does not accurately supply a negative voltage, so only positive voltages should be used. This is not a big issue since the leads can be switched to apply the voltage in the opposite direction.

\subsection{CONTRIBUTIONS}

Flow profiles have a large impact on mixing applications in T-mixers. Parabolic profiles can lead to uneven mixing because different parts of the flow come in contact at different times. It is desired to have a completely uniform flow and electro-kinetic flows tend to do a better job at delivering uniform velocity profiles.

Previous work at Cal Poly has been done to make quantum dots using microfluidics to control the size and the fluorescence of the particles [47].

Pressure-driven flow leads to a large distribution of sizes and fluorescence in one sample. Electro-kinetic plug shaped flow profile could lead to a more even mix giving better control of the nucleation and growth process and a smaller distribution of sizes and fluorescence. 
Future work could be done to characterize mixing systems with pressure-driven and electro-kinetically drive flows. Eventually work can be done to integrate quantum dot synthesis with electro-kinetic flow. 


\section{REFERENCES}

[1] G. V. Casquillas, "Tutorials," Elveflow, [Online]. Available: http://www.elveflow.com/. [Accessed 27 November 2013].

[2] R. Savage, "MEMS Design, Cantilever, Accelerometer," in MATE 430, San Luis Obispo, 2013.

[3] N.-T. Nguyen and S. T. Wereley, Fundamentals and Applications of Microfluidics, Norwood: Artech House, Inc., 2002.

[4] H.-S. Chuang, "Microfluidics for Life Science and Chemistry Microfilters \& Microseparators," in Fundamentals, Characterizations and Applications of Microscale Fluids, 2012.

[5] H.-S. Chuang, "Microfluidics for Life Science and Chemisty: Microneedles \& MicroMixers," in Fundamentals, Characterizationa and Applications of Microscale Fluids, 2012.

[6] University of Notre Dame, "From the Director," Center for Microfluidics and Medical Diagnostics, 2004. [Online]. Available:

http://microfluidics.nd.edu/about/. [Accessed 27 November 2013].

[7] T. Srinivasan, "Microfluidics for DNA Analysis," in EE C 245/.

[8] R. C. Lo, "Application of Microfluidics in Chemical Engineering," Chemical Engineering \& Process Techniques, vol. 1, no. 1002, pp. 1-3, 2013.

[9] L. Kang, B. G. Chung, R. Langer and A. Khademhosseini, "Microfluidics for drug discovery and developement: From targer selection to production lifecycle management," Drug Discovery Today, vol. 13, pp. 2-13, 2008.

[10] E. M. Miller, S. Freire and A. R. Wheeler, "Proteomics in Microfluidic Devices," pp. 1749-1758.

[11] Dow Corning, "SYLGARD® 184 SILICONE ELASTOMER KIT," Dow Corning Corporation, 2013. [Online]. Available:

http://www.dowcorning.com/applications/search/default.aspx?r=131en. [Accessed 28 November 2013].

[12] S. o. M. s. a. Engineering, "Ceramics," The University of New South Wales, [Online]. Available:

http://www.hsctut.materials.unsw.edu.au/Ceramics/ceramics1a.htm. 
[Accessed 4 June 2014].

[13] Polymer Science Learning Center, "Poly(methyl methacrylate)," The University of Southern Mississippi: Department of Polymer Science, 2005. [Online]. Available: http://pslc.ws/mactest/pmma.htm. [Accessed 9 December 2014].

[14] TOPAS, "TOPAS COC Polymers," TOPAS Advanced Polymers, April 2011. [Online]. Available: http://www.topas.com/products/topas-coc-polymers. [Accessed 9 December 2014].

[15] Cidra, "Flow Cell Materials," CiDRA® Precision Services, LLC, 2012. [Online]. Available: http://www.cidraprecisionservices.com/flow-cellmaterials.html. [Accessed 9 December 2014].

[16] R. Savage, Writer, MATE 225 Microfluidic Project. [Performance]. 2012.

[17] T. A\&M, "ALChemE: Active Learning in Chemical Engineering," Chemical Engineering Department Online Curriculum Reform Project, [Online]. Available: http://alcheme.tamu.edu/?page_id=1088. [Accessed 28 November 2013].

[18] G. K. Butt, Physics and Chemistry of Interfaces, Weinheim: Wiley, 2006.

[19] B. K. a. L. M. Weiland, "Experimental investigation of the streaming potential hypothesis for ionic polymer transducers in sensing," IOP science, vol. 22, no. 3, 2013.

[20] N. Anderson, "Bond Strength Characterization of SU-8 to SU-8 for Fabricating Microchannels of an Electrokinetic Microfluidic Pump," California Polytechnic State University, San Luis Obispo, 2012.

[21] D. J. Laser, "Electrokinetics and Electroosmotic Flow," Design Division, Department of Mechanical Engineering,Stanford University , [Online]. Available:

http://micromachine.stanford.edu/ dlaser/research_pages/electrokinetic s_and_eof.html. [Accessed 28 November 2013].

[22] The Engineering Toolbox, "Types of Fluid Flowmeters," The Engineering Toolbox, [Online]. Available: http://www.engineeringtoolbox.com/flowmeters-d_493.html. [Accessed 28 November 2013].

[23] Velocimetry, "Principles," Velocimetry Technology (P) Limited, [Online]. Available: http://velocimetry.net/principle.htm. [Accessed 1 June 2014]. 
[24] Michigan State University, "Molecular Tagging Velocimetry (MTV)," College of Engineering, [Online]. Available:

http://www.egr.msu.edu/tmual/MTV.html. [Accessed 1 June 2014].

[25] "How does a confocal microscope work?," [Online]. Available:

http://www.physics.emory.edu/ weeks/confocal/. [Accessed 28

November 2013].

[26] LabSmith, "SVM340 Synchronized Video Microscope User's Guide," 2012. [Online]. Available: http://labsmith.com/support/svm340-synchronizedvideo-microscope/. [Accessed 9 December 2014].

[27] W. Zheng, Z. Wang, W. Zhang and X. Jiang, "A simple PDMS-based microfluidic channel design that removes bubbles for long-term on-chip culture of mammalian cells," Lab Chip, pp. 2906-2910, 2010.

[28] Translume, "Microfluidic Chips with Cross-Shaped Channel," Translume, 2014. [Online]. Available: http://www.translume.com/index.php/webstore/microfluidic-chips/cross-channel-chips. [Accessed 8 October 2014].

[29] Translume, "Microfluidic Chips with Straight Channel," Translume, 2014. [Online]. Available: http://www.translume.com/index.php/webstore/microfluidic-chips/straight-channel-chips. [Accessed 8 October 2014].

[30] Cidra, "Catalog Microfluidic Chips," CiDRA® Precision Services, LLC, 2012. [Online]. Available: http://www.cidraprecisionservices.com/custom-flow-cells.html. [Accessed 8 October 2014].

[31] Cidra, "Flow Cell Cleaning Protocol," CiDRA® Precision Services, LLC, 2012. [Online]. Available: http://www.cidraprecisionservices.com/flowcell-cleaning-protocol.html. [Accessed 8 October 2014].

[32] LabSmith, "Microfluidic Fluid Control \& Connectors," LabSmith, Inc., 2012. [Online]. Available: http://labsmith.com/support/microfluidics/. [Accessed 30 October 2014].

[33] Cidra, "PDMS Wetting Agent 564," CiDRA® Precision Services, LLC, 2012. [Online]. Available: http://www.cidraprecisionservices.com/surfactantpdms-microfluidics.html. [Accessed 6 October 2014].

[34] Life Technologies, "FluoSpheres ${ }^{\circledR}$ Polystyrene Microspheres, $10 \mu \mathrm{m}$, bluegreen fluorescent (430/465), for blood flow determination," Thermo 
Fisher Scientific Inc., 2014. [Online]. Available:

http://www.lifetechnologies.com/order/catalog/product/F8830.

[Accessed 7 October 2014].

[35] Life Technologies, "FluoSpheres® Biotin-Labeled Microspheres, $1.0 \mu \mathrm{m}$, yellow-green fluorescent (505/515), 1\% solids," Thermo Fisher Scientific Inc., 2014. [Online]. Available:

http://www.lifetechnologies.com/order/catalog/product/F8768.

[Accessed 7 October 2014].

[36] Life Technologies, "FluoSpheres $\AA^{\circledR}$ Carboxylate-Modified Microspheres, 1.0 $\mu \mathrm{m}$, red fluorescent (580/605), 2\% solids," Thermo Fisher Scientific Inc., 2014. [Online]. Available:

http://www.lifetechnologies.com/order/catalog/product/F8821.

[Accessed 2014 October 2014].

[37] Life Technologies, "FluoSpheres ${ }^{\circledR}$ Carboxylate-Modified Microspheres, 2.0 $\mu \mathrm{m}$, yellow-green fluorescent (505/515), $2 \%$ solids," Thermo Fisher Scientific Inc., 2014. [Online]. Available:

http://www.lifetechnologies.com/order/catalog/product/F8827. [Accessed 13 February 2014].

[38] Life technologies, "FluoSpheres $®$ Polystyrene Microspheres, $1.0 \mu \mathrm{m}$, red fluorescent (580/605), for tracer studies," 2014. [Online]. Available: https://www.lifetechnologies.com/order/catalog/product/F13083?ICID=s earch-f13083. [Accessed 1 March 2014].

[39] New Era Pump Systems Inc., "NE-1000 Series of Programmable Syringe Pumps," 27 August 2014. [Online]. Available: syringepump.com. [Accessed 16 January 2015].

[40] Harvard Apparatus, "Model '11' Plus Syringe Pump User's Manual," [Online]. Available: www.instechlabs.com/Pumps/syringe/. [Accessed 15 January 2015].

[41] LabSmith, "Pressure Sensor Starter Package," LabSmith, 2014. [Online]. Available: http://products.labsmith.com/pressure-sensor-starterpackage/\#.VIm4ZXu2oeY. [Accessed 20 October 2014].

[42] LabSmith, "LabSmith uProcess System User Guide," 2013. [Online]. Available: www.labsmith.com. [Accessed 15 October 2014].

[43] R. Savage, "Micro-Systems Technology: Microfluidics," in MATE550, San Luis Obispo, 2014. 
[44] H. Bruus, Theoretical Microfluidics, New York: Oxford University Press, 2008.

[45] C. L. Berli, M. V. Piaggio and J. A. Deiber, "Theoretical relation between the tube zeta potential and the background electrolyte composition in capillary electrophoresis," Colloid Polymer Science, vol. 128, pp. 21-24, 2004.

[46] B. J. Kirby and E. F. Hasselbrink, "Zeta potential of microfluidic substrates," Electrophoresis, vol. 25, pp. 203-213, 2004.

[47] H. Lafferty and J. Hoadley, "Development of High Precision Quantum Dot Synthesis Method Utilizing a microfluidic Reactor and In-Line Fluoresence Cell," Cal Poly, San Luis Obispo, 2013.

[48] ePlastics, "Chemical Resistance of Plexiglass Acrylic," Ridout Plastics Co. Inc., 2014. [Online]. Available:

www.eplastics.com/Plastic/Plastics_Library/Chemical-Resistance-ofPlexiglass-Acrylic. [Accessed 3 November 2014]. 


\section{APPENDICES}

\section{A. PDMS Wetting Agent 564 by CIDRA PRECISION SERVICES}

A study was conducted to determine if PDMS Wetting Agent 564 by Cidra

Precision Services increases wettability of surfaces. Wettability is measured by the contact angle between a surface and a drop on the surface. The solutions used in this experiment were water based so a surface that does not wet well, has a wetting angle greater than $90^{\circ}$, is said to be hydrophobic, while a surface that does wet well, has a wetting angle less than $90^{\circ}$, is said to be hydrophobic. Cidra recommends a concentration between $0.001 \%$ and $0.1 \%$.

A 2-way factorial treatment structure was used, varying the concentration of wetting agent at 4 levels $(0 \%, 0.001 \%, 0.01 \%, 0.1 \%)$ and the surface material at 2 levels (PDMS, PS). PMDS was used because this solution is specifically made for PDMS but can be used for other polymers and PS was used to represent the other polymer channel. The design structure was a completely randomized design (CRD). Randomized testing of treatment order was done using JMP Full Factorial Design. Two replicates were recommended by a pilot study to achieve a power of 0.90 , but 6 replicates were done for more power. There was no blocking or nesting.

Each droplet placed on the surface defined the experimental unit. The average contact angle was calculated from the right and left contact angle measured on the AST VCA Optima goniometer. Other direct controls were also taken. A piece of tape was placed on the PDMS surface prior to testing to keep dirt particles from affecting surface properties that might change the contact angle. In addition, 
static charge buildup was known to be an issue with PS. Before each use, the PS surface was cleaned with IPA and brushed with aluminum foil to minimize any built-up charge.

The experiment was carried out by first preparing the variables. Solutions with the appropriate concentration of wetting agent with DI water were mixed on a Cole-Parmer vortex mixer. The PDMS was mixed in a 10:1 ratio and placed under vacuum to remove any bubbles then poured into a petri dish and placed in an oven at $70^{\circ} \mathrm{C}$ for 2 hours to cure. This was kept clean with scotch tape. A petridish was used as the PS surface sample.

To obtain measurements, the surface sample was placed on the platform on the goniometer and the appropriate solution was loaded into the syringe. A $1 \mu \mathrm{L}$ drop was ejected from the droplet and the surface was raised to the droplet. An image was taken and 5 points were placed along the edge of the droplet. The goniometer software was then able to calculate the contact angle.

JMP statistical software with a 0.05 level of significance was used to analyze the data of this experiment. Multiple comparisons were performed and the FWER was controlled as appropriate. Bonferroni's adjustment was used to control the FWER of the global F-tests. Tukey's adjustment was used to control the FWER of the overall pairwise comparison. Dunnett's adjustment was used to control the FWER of the concentrations comparison. No adjustment was used for the $\Gamma=\mu_{0 \%}-\frac{\mu_{0.001 \%}+\mu_{0.01 \%}+\mu_{0.1 \%}}{3}$ contrast. 
An outlier was found for one of the measurements for $0.01 \%$ wetting agent on the PS surface, most likely from static charge build up which could not always be avoided. With a p-value of 0.4246 , the interaction between concentration and surface material was not found to be statistically significant. With $p$-values of less than 0.0001 for both concentration and surface material, the main effects were found to be statistically significant. According to Tukey's overall pairwise test, the smallest contact angle can be achieved with $0.01 \%$ and $0.1 \%$ concentrations for both PDMS and PS surfaces. According to Dunnett's pairwise test, the $0.01 \%$ and $0.1 \%$ concentrations are statistically significantly different from $0 \%$, but $0.001 \%$ is not with $p$-values of 0.0032 , less than 0.0001 , and 0.9967 respectively. According to the $\Gamma=\mu_{0 \%}-\frac{\mu_{0.001 \%}+\mu_{0.01 \%}+\mu_{0.1 \%}}{3}$ contrast test, with a $p$-value of 0.0003, the wetting agent did make a statistically significant contact angle. Finally, it is concluded that PDMS Wetting Agent 564 by Cidra Precision Services does make a statistically significant contact angle at $0.01 \%$ and $0.1 \%$ on both PDMS and PS surfaces. 


\section{B. LABSMITH SYNCHRONIZED VIDEO MICROSCOPE}

\section{Camera Configuration}

Camera Description

Untitled LabSmith Camera Module

Imager Properties

Monochrome

Color

Scan:

Interlaced

- Progressive

Output:

Analog

○ Digital

\begin{tabular}{|c|c|c|c|c|}
\hline \multirow{3}{*}{ Array Size: } & \multicolumn{2}{|c|}{ Horizontal } & \multicolumn{2}{|l|}{ Vertical } \\
\hline & 4.2 & mm & 3.06999999 & \\
\hline & 720 & pixels & 480 & \\
\hline
\end{tabular}

Illumination Type

(-) LED Ring Illuminator Only

Epifluorescence

Frame triggering

(-) Camera is self triggered

Triggering needed

Note: these settings are used to configure the SVM340 and uScope software. If you change them, please make sure that these settings describe your hardware. Changes will be adopted the next time you turn on your SVM340 and restart uScope. 


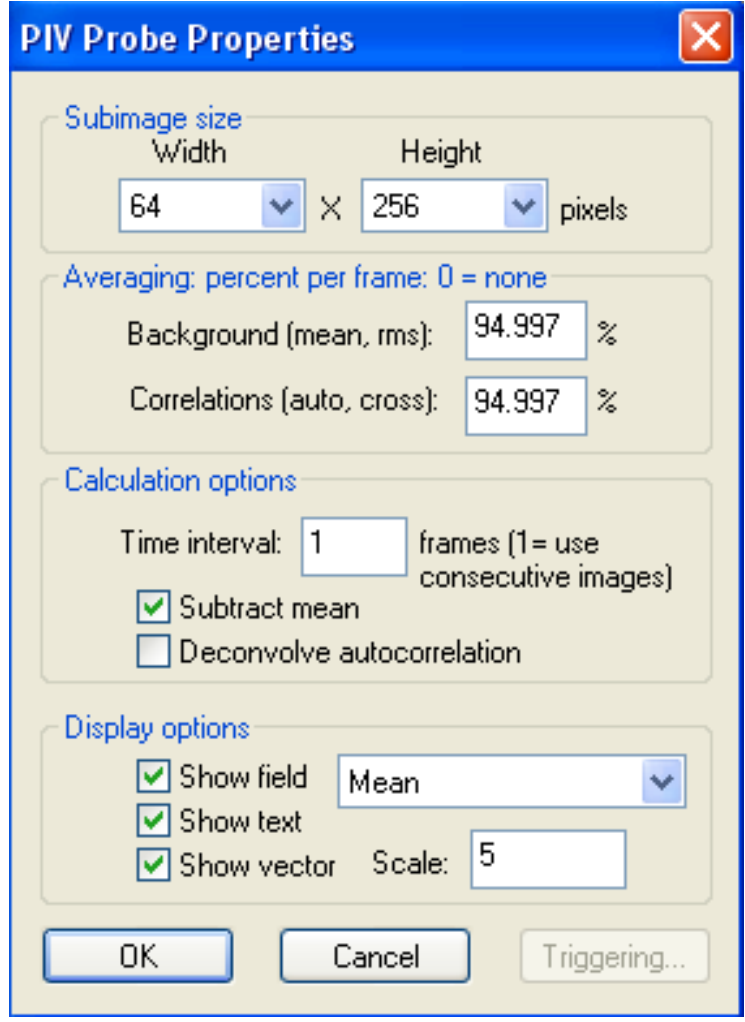




\section{LABSmith High Voltage SeQuenceR}

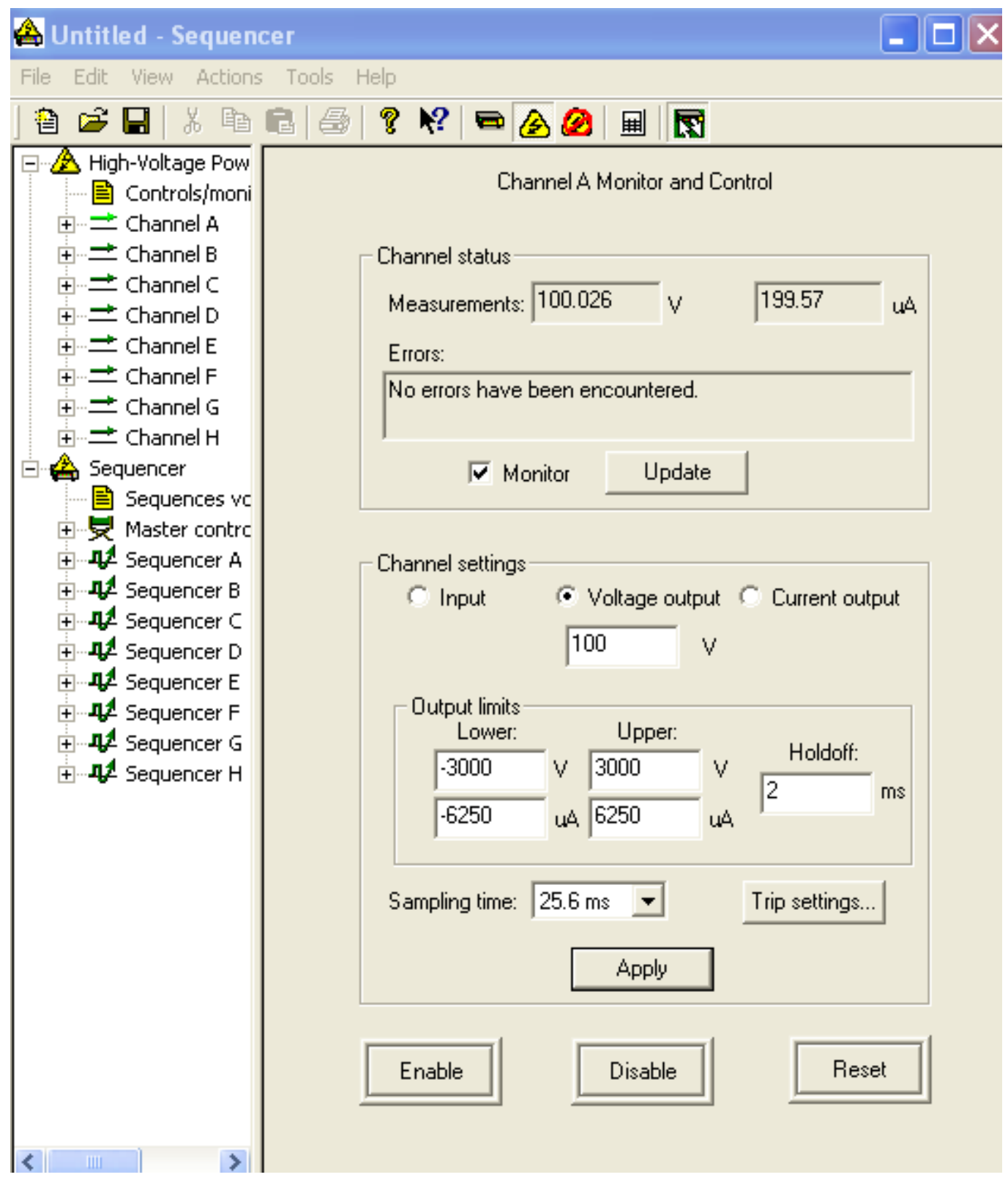


D. Modeling Pressure-Driven flow at Varying Flow Rates

\section{$1.0 \mu \mathrm{L} / \mathrm{min}$}

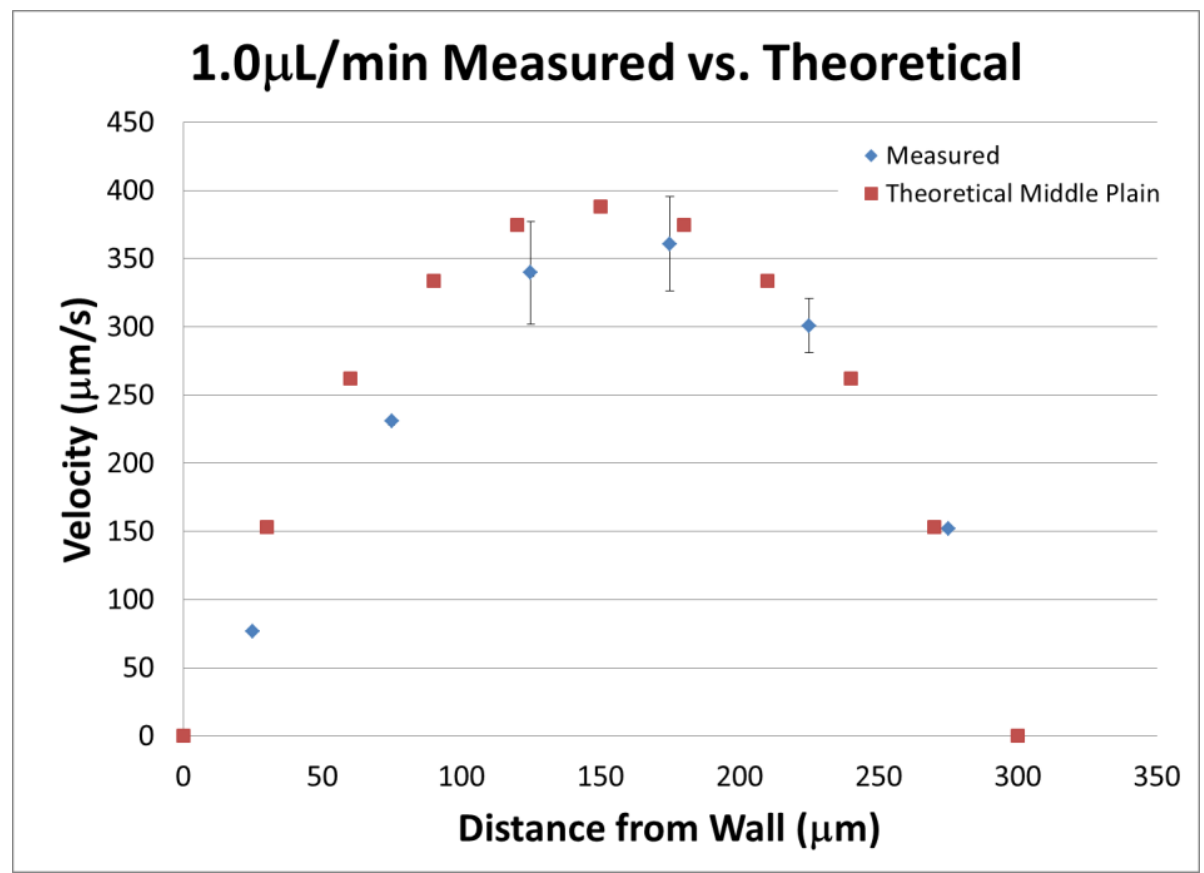

$1.5 \mu \mathrm{L} / \mathrm{min}$

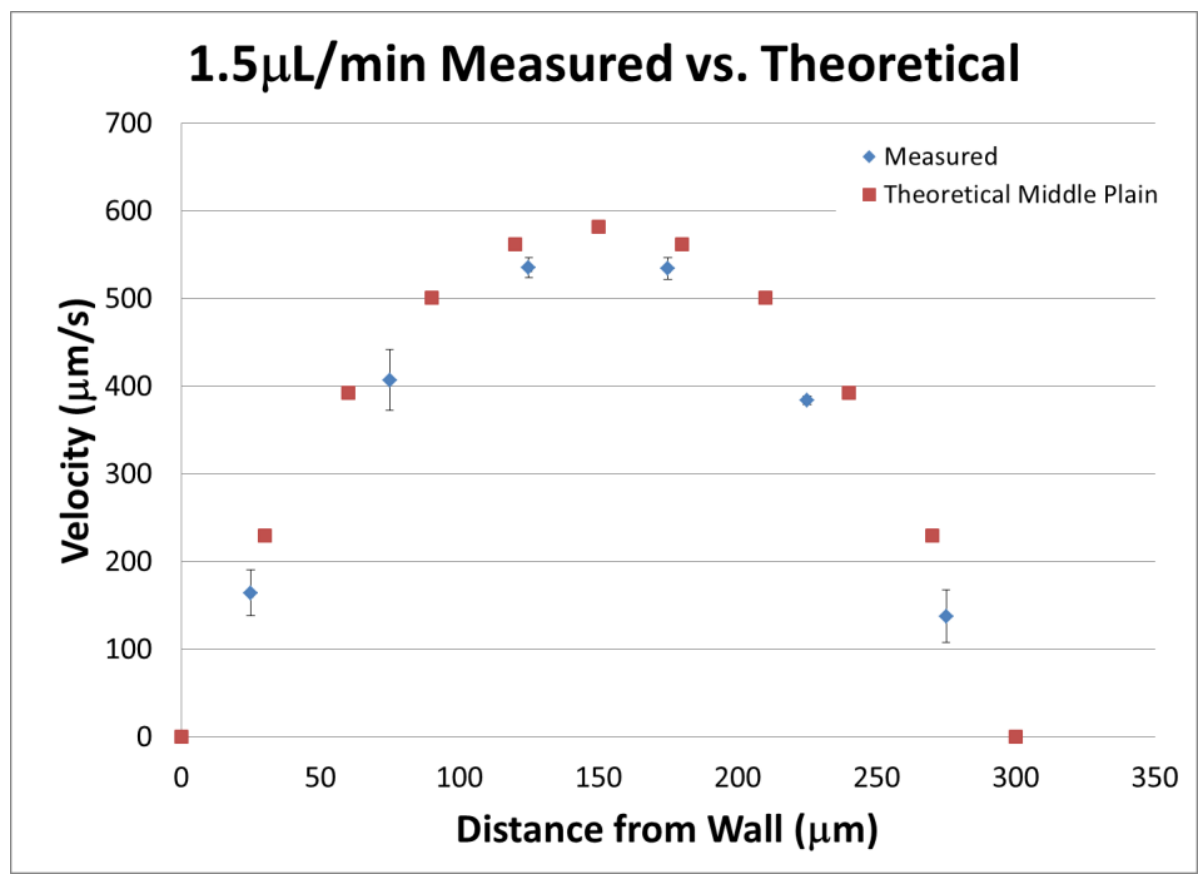




\section{$2.0 \mu L / m i n$}

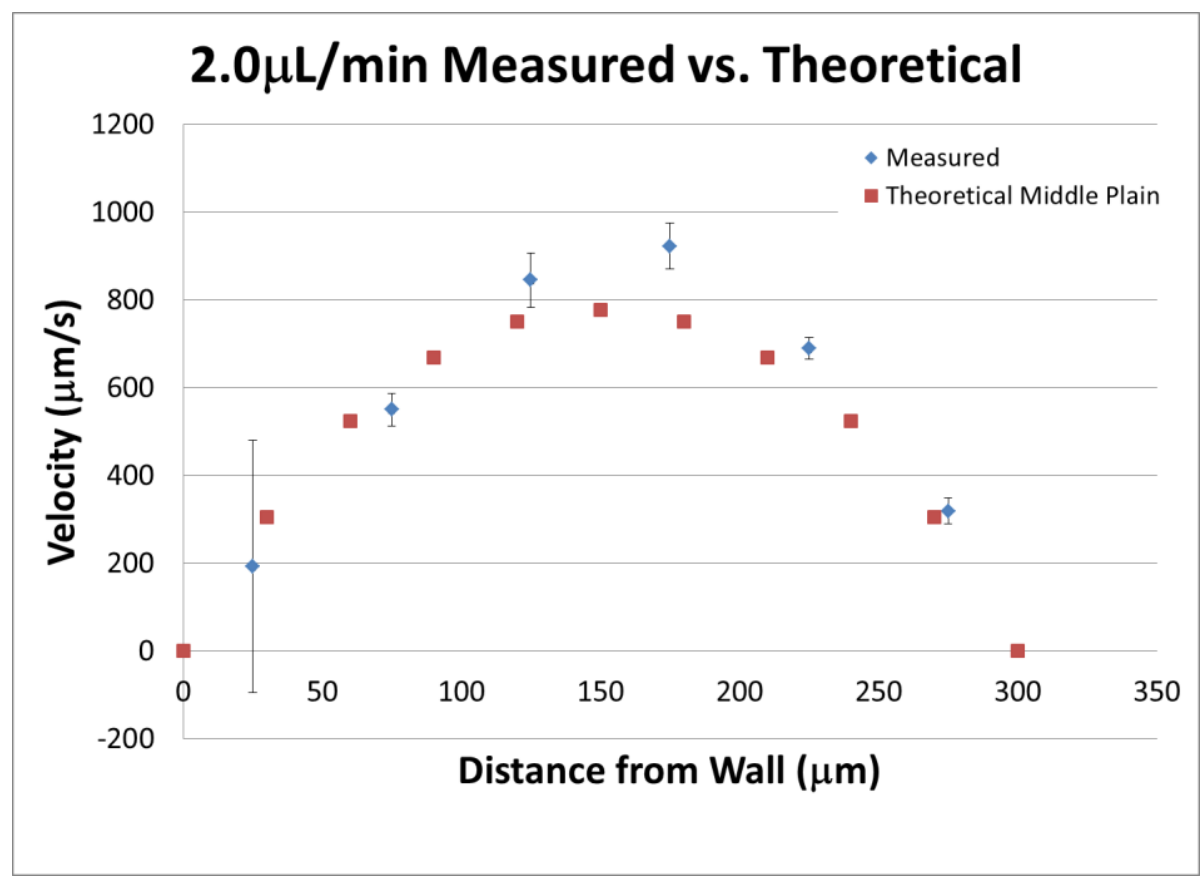

$3.0 \mu \mathrm{L} / \mathrm{min}$

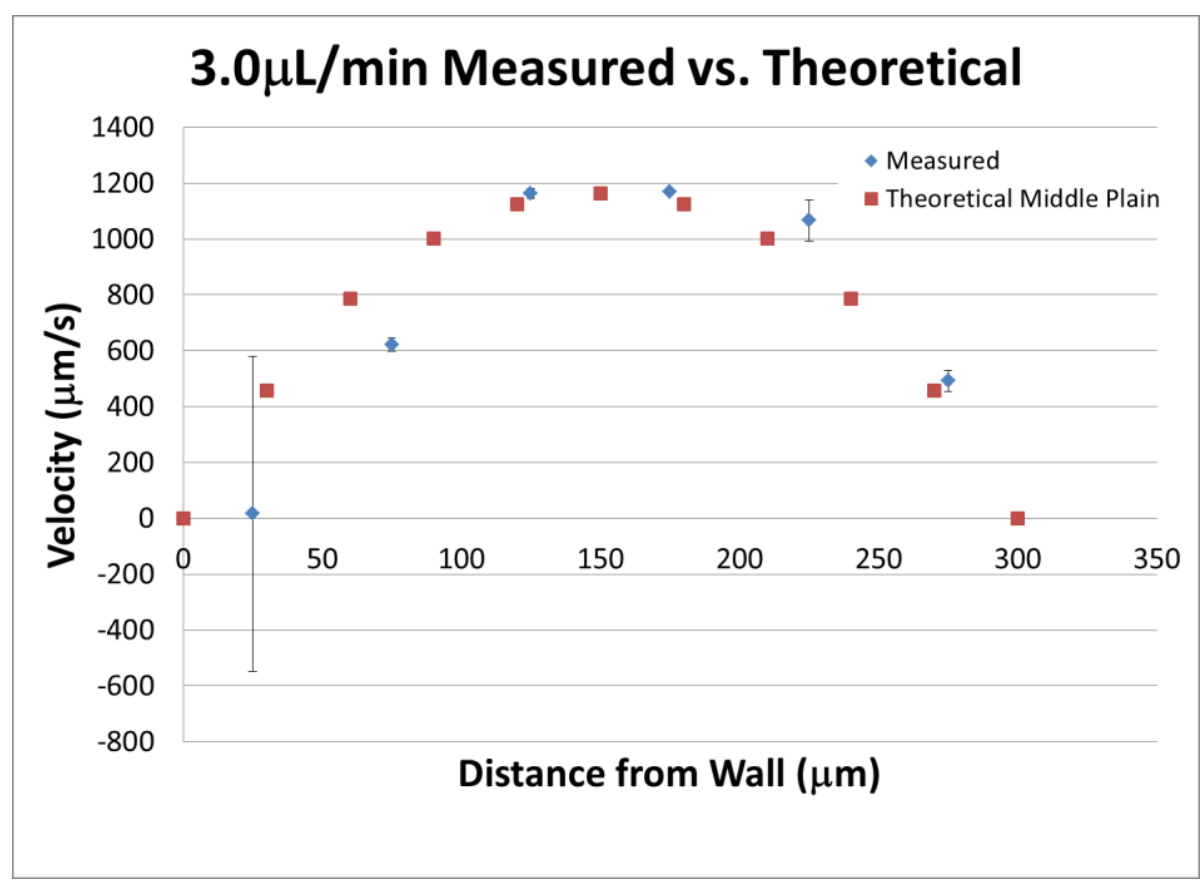




\section{$3.5 \mu L / m i n$}

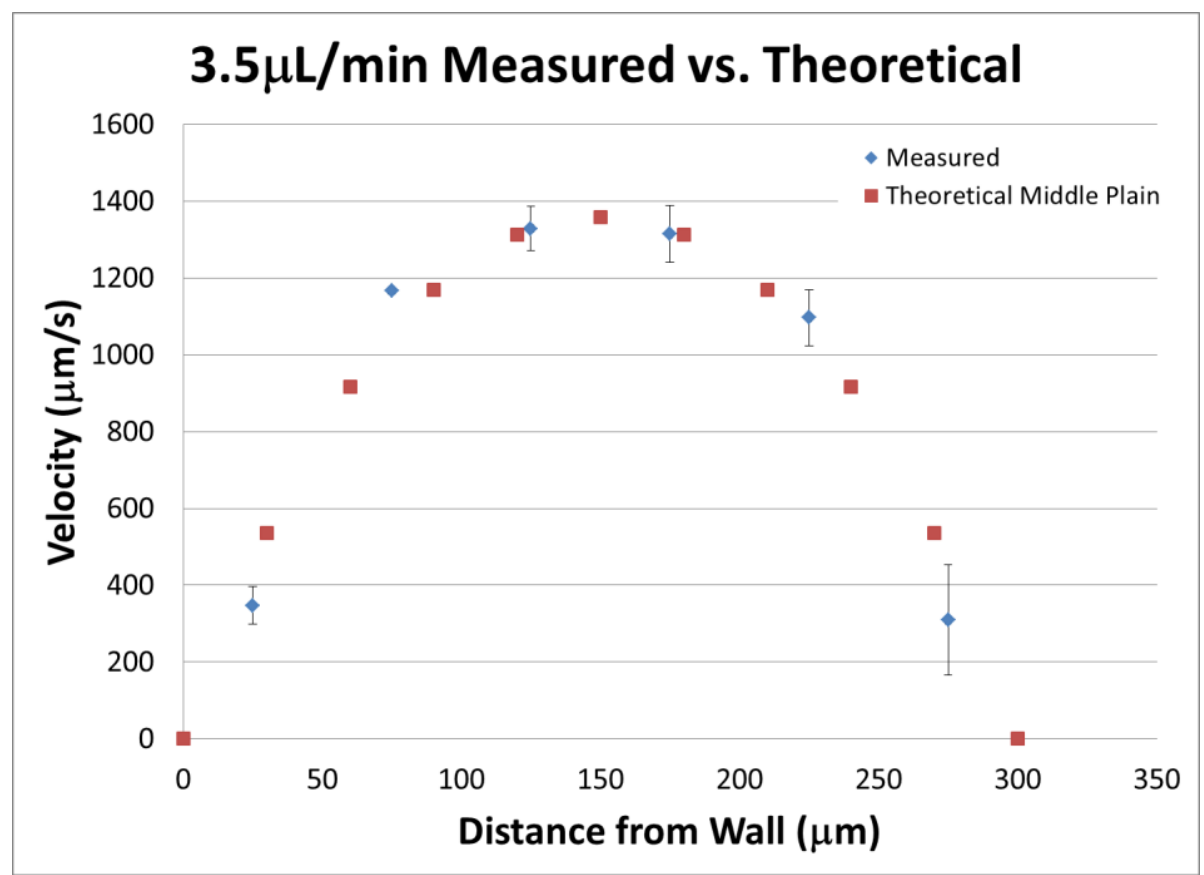

$4.0 \mu L / m i n$

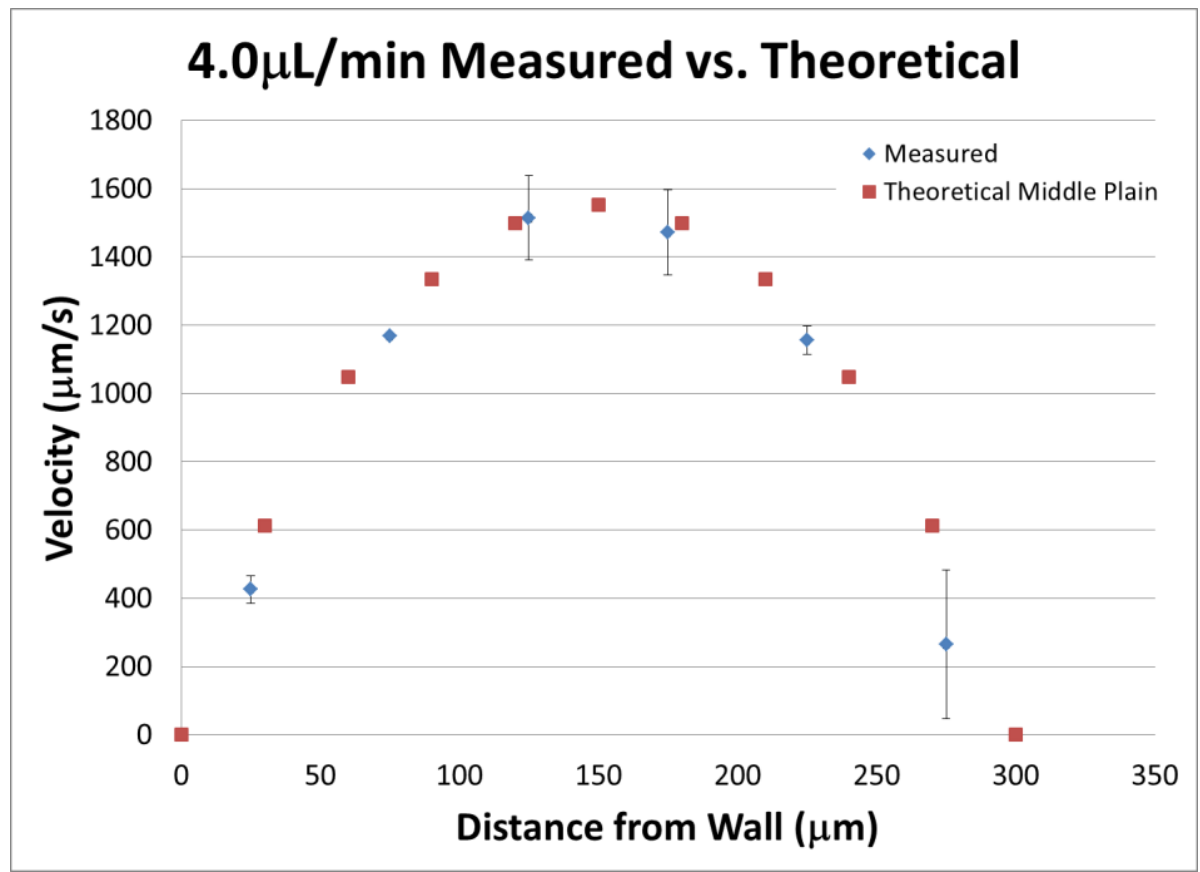

